\title{
The mean magnetic field modulus of Ap $\operatorname{stars}^{\star, \star \star}$
}

\author{
G. Mathys ${ }^{1}$, S. Hubrig ${ }^{1,2}$, J.D. Landstreet ${ }^{3, \star \star \star}$, T. Lanz ${ }^{4, \dagger}$, and J. Manfroid ${ }^{5}$ \\ 1 European Southern Observatory, Casilla 19001, Santiago 19, Chile \\ 2 University of Potsdam, Am Neuen Palais 10, D-14469 Potsdam, Germany \\ 3 Department of Astronomy, University of Western Ontario, London, Ontario N6A 3K7, Canada \\ 4 Astronomical Institute, Utrecht University, Princetonplein 5, P.O. Box 80000, NL-3508 TA Utrecht, The Netherlands \\ 5 Institut d'Astrophysique, Université de Liège, avenue de Cointe 5, B-4000 Liège, Belgium
}

Received July 19; accepted September 9, 1996

\begin{abstract}
We present new measurements of the mean magnetic field modulus of a sample of Ap stars with spectral lines resolved into magnetically split components. We report the discovery of 16 new stars having this property. This brings the total number of such stars known to 42 . We have performed more than 750 measurements of the mean field modulus of 40 of these 42 stars, between May 1988 and August 1995. The best of them have an estimated accuracy of $25-30 \mathrm{G}$. The availability of such a large number of measurements allows us to discuss for the first time the distribution of the field modulus intensities. A most intriguing result is the apparent existence of a sharp cutoff at the low end of this distribution, since no star with a field modulus (averaged over the rotation period) smaller than $2.8 \mathrm{kG}$ has been found in this study. For more than one third of the studied stars, enough field determinations well distributed throughout the stellar rotation cycle have been achieved to allow us to characterize at least to some extent the variations of the field modulus. These variations are often significantly anharmonic, and it is not unusual for their extrema not to coincide in
\end{abstract}

Send offprint requests to: G. Mathys

* Based on observations collected at the European Southern Observatory (La Silla, Chile; ESO programmes Nos. 43.7-004, 44.7-012, 49.7-030, 50.7-067, 51.7-041, 52.7-063, 53.7-028, 54.E0416, and 55.E-0751), at the Observatoire de Haute-Provence (Saint-Michel-l'Observatoire, France), at Kitt Peak National Observatory, and at the Canada-France-Hawaii Telescope.

** Tables 2, 3, and 4 are also available in electronic form at the CDS via anonymous ftp to

cdsarc.u-strasbg.fr (130.79.128.5) or via

http://cdsweb.u-strasbg.fr/Abstract.html

$\star \star \star$ Visiting Astronomer, Canada-France-Hawaii Telescope operated by the National Research Council of Canada, the Centre National de la Recherche Scientifique de France and the University of Hawaii.

$\dagger$ Visiting Astronomer, Kitt Peak National Observatory, National Optical Astronomy Observatories, operated by AURA Inc., under contract with the National Science Foundation. phase with the extrema of the longitudinal field (for the few stars for which enough data exist about the latter). This, together with considerations on the distribution of the relative amplitude of variation of the studied stars, supports the recently emerging evidence for markedly nondipolar geometry and fine structure of the magnetic fields of most Ap stars. New or improved determinations of the rotation periods of 9 Ap stars have been achieved from the analysis of the variations of their mean magnetic field modulus. Tentative values of the period have been derived for 5 additional stars, and lower limits have been established for 10 stars. The shortest definite rotation period of an Ap star with magnetically resolved lines is 3.4 , while those stars that rotate slowest appear to have periods in excess of 70 or 75 years. As a result of this study, the number of known Ap stars with rotation periods longer than 30 days is almost doubled. We briefly rediscuss the slow-rotation tail of the period distribution of Ap stars. This study also yielded the discovery of radial velocity variations in 8 stars. There seems to be a deficiency of binaries with short orbital periods among Ap stars with magnetically resolved lines.

Key words: stars: chemically peculiar — stars: magnetic fields - stars: rotation

\section{Introduction}

Since the discovery, almost 50 years ago, of a magnetic field in the Ap star 78 Vir (Babcock 1947), until recently, the vast majority of the investigations of Ap star magnetic fields have been devoted to the determination of their mean longitudinal magnetic field (see e.g. the reviews of Mathys 1989 and Landstreet 1992). The latter, to which one often refers more briefly as the longitudinal field ${ }^{1}$, is

$\overline{1}$ In the past, it was also often called effective field. 
the line-intensity weighted average over the visible stellar hemisphere of the line-of-sight component of the magnetic vector. It is diagnosed from the observation of circular polarization in spectral lines. From studies of this field moment, and in particular of its variation with stellar rotation, a general picture of the magnetic fields of Ap stars could be obtained. As a result, it is now well established that those fields have a large-scale organization, which in most cases bears some resemblance to a dipole whose axis is inclined with respect to the stellar rotation axis. The brightness and abundance inhomogeneities of the stellar surface appear grossly related to the field geometry. Thus the study of the latter should prove essential for the understanding of the origin of the former, and more generally of the physical processes at work in the atmospheres not only of the Ap stars, but also probably of many upper mainsequence stars. However, the derivation of the magnetic field geometry cannot be achieved from the consideration of the mean longitudinal field alone.

One subgroup of the Ap stars lend themselves particularly well to more detailed studies of their magnetic fields: these are the stars whose spectral lines, when observed (in unpolarized light) at sufficiently high dispersion, are resolved into several magnetically split components. In these stars, one can determine in a straightforward, mostly approximation-free, model-independent manner, and with particularly great precision, the mean magnetic field modulus, or in short the (mean) field modulus. This quantity, which in previous works was often called the surface field, is the line-intensity weighted average over the visible stellar hemisphere of the modulus of the magnetic vector.

It had been realized 25 years ago already that by combining the knowledge of the variations of the field modulus and of the longitudinal field throughout the stellar rotation cycle, one could derive much better constraints on the field structure than from the consideration of the longitudinal field alone. By 1972, this approach had been applied successfully to 4 stars: HD 65339 (Huchra 1972), HD 126515 (Preston 1970), HD 137909 (Wolff \& Wolff 1970), and HD 215441 (Preston 1969a). Preston (1971a) had discovered 5 more stars with magnetically resolved lines (HD 12288, HD 81009, HD 165474, HD 188041, and HDE 335238). But no attempts had been made to measure their mean field modulus throughout their rotation cycle until we started the project reported here.

Renewed interest in the modeling of the geometric structure of Ap star magnetic fields has arisen in the last few years, thanks to the technological progress made both in astronomical instrumentation (in particular, detectors) and in computers. Accordingly, it has become possible to collect astronomical data of unprecedented accuracies (making possible e.g. fine studies of the profiles of polarized spectral lines), which can be modeled more realistically than ever before. For instance, Landstreet and his collaborators have carried out refined studies of the magnetic field geometry of HD 65339 (Landstreet 1988) and of HD 215441 (Landstreet et al. 1989), by simultaneously modeling their unpolarized spectral line profiles and the variations of their longitudinal field. Mathys (1995a, b) has analyzed the circularly polarized line profiles of a sample of Ap stars observed throughout their rotation cycle using the moment technique that he has developed (Mathys 1988). Leroy et al. (1995) have derived models of the fields of the stars HD 62140, HD 71866, and HD 137909, from their observations of broadband linear polarization along the rotation periods of these stars. These are but a few examples of the recently published works in the field.

Still, the very favourable circumstances presented by the stars with resolved magnetically split lines for the diagnosis of the field geometry are far from having been fully exploited. By the end of the 1980's, only 4 of the 9 stars of this kind known in the early 1970's had been studied in detail. A few additional such stars had been discovered in isolated studies: HD 200311 (Preston, cited by Adelman 1974), HD 201601 (a somewhat marginal detection reported by Scholz 1979), and HD 187474 (Didelon 1987). But there had been no systematic study of these stars, nor attempt to find more of them. Therefore, it seemed appropriate to undertake a large-scale project of extensive investigation of the Ap stars with resolved magnetically split lines.

This programme has been going on for more than 5 years now. By its very nature, in particular because a significant number of the studied stars have periods (much) longer than the time span during which we have followed them, it is not completed yet. However, it appears justified to report the results obtained so far, in view of their quantity and of their importance. Indeed, 29 new Ap stars with resolved magnetically split lines have been found, and more than 750 measurements of the mean magnetic field modulus of such stars have been performed. The results presented in this paper correspond to the observations that have been performed for this programme until end of August 1995.

Some partial, preliminary reports on this project have already been given in previous publications. Mathys (1990; hereafter referred to as Paper I) had discussed the physics of the formation of the spectral line Fe II $\lambda 6149.2$, from which we diagnose the field modulus. He also had reported the discovery of magnetically resolved lines in 3 stars: HD 55719, HD 94660, and HD 116458, and confirmed Scholz's (1979) suspicion for the presence of resolved lines in HD 201601. Mathys \& Lanz (1992; Paper II) had announced the presence of resolved magnetically split lines in 6 additional stars: HD 2453, HD 9996, HD 18078, HD 50169, HD 137949, and HD 192678. They had also discussed the relative magnetic intensification of the Fe II lines $\lambda 6147.7$ and $\lambda 6149.2$. The discovery of 4 more stars with magnetically resolved lines (HD 14437, HD 110066, HD 116114, and HD 134214) had first been reported by Mathys et al. (1993; Paper III). 


\section{The mean magnetic field modulus}

The determinations of the mean magnetic field modulus presented in this paper rely on the measurement of the wavelength shift between the magnetically split components of the line Fe II $\lambda 6149.2$, in high-resolution spectra recorded in unpolarized light. The line Fe II $\lambda 6149.2$ has a particularly simple Zeeman pattern, consisting of two $\pi$ components, one $\sigma_{+}$component and one $\sigma_{-}$component. Each $\sigma$ component coincides with one of the $\pi$ components. This pattern, as observed in the star HD 94660, is shown in Fig. 1, together with those of the neighbouring lines Fe II $\lambda 6147.7$ (a pseudo-quadruplet) and Cr II $\lambda 6147.1$ (a pseudo-triplet). For comparison, we have also plotted the spectrum of the A0p SrCr star HD 133792 (Paper I), which has sharp unresolved lines (the line profiles recorded in this star are mostly identical to the instrumental profile: its projected equatorial velocity must be significantly lower than $3 \mathrm{~km} \mathrm{~s}^{-1}$ ).

A Zeeman doublet like Fe II $\lambda 6149.2$ arises from a transition between two levels having a total angular momentum quantum number $J=1 / 2$, of which one has a Landé factor equal to zero (that is, this level is unsplit in a magnetic field). In such a doublet, the shift $\left(\lambda_{\mathrm{r}}-\lambda_{\mathrm{b}}\right)$ between the wavelengths of the red and blue components is related to the mean magnetic field modulus $\langle H\rangle$ through the relation:

$\lambda_{\mathrm{r}}-\lambda_{\mathrm{b}}=g \Delta \lambda_{\mathrm{Z}}\langle\mathrm{H}\rangle$.

$\Delta \lambda_{\mathrm{Z}}=\mathrm{k} \lambda_{0}^{2}$, where $\lambda_{0}$ is the nominal wavelength of the transition (in the absence of a magnetic field, that is, for the line under consideration, $\lambda_{0}=6149.258 \AA$ ), and $k=4.6710^{-13} \AA^{-1} \mathrm{G}^{-1} . g$ is the Landé factor of the split level of the transition ( $g=2.70$; Sugar \& Corliss 1985). For a Zeeman doublet, Eq. (1) is strictly valid under quite general conditions. The only approximations underlying it are that the populations of the magnetic states pertaining to the same atomic level follow Boltzmann's statistics and that the Doppler effect due to stellar rotation is negligible (see Mathys 1989 for details). The high densities in the photospheres of the Ap stars coupled to the very small energy differences between the magnetic states guarantee the validity of the first assumption. It receives further support from the following empirical argument: significant departures from this approximation would show through as spectral line asymmetries, both in unpolarized light and in circular polarization: such asymmetries are not observed. On the other hand, in a number of the stars studied in this paper, Doppler distortions of the split line components are observed. But this does not question the validity of the second approximation above: more explicitly, the latter states that the rotational Doppler effect is small compared to the magnetic splitting, which is also the condition of magnetic resolution of the lines.

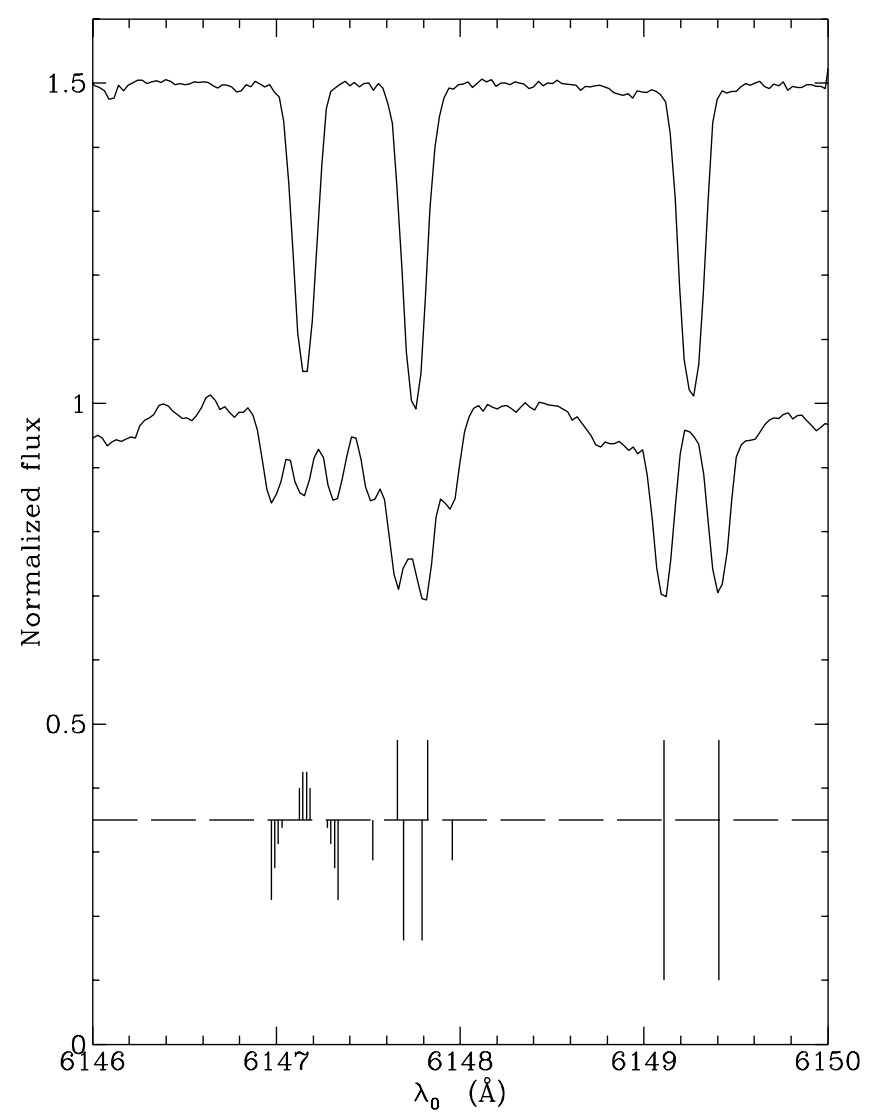

Fig. 1. Portion of the spectra of HD 133792 (shifted in intensity by 0.5 ; unresolved lines) and of HD 94660 containing the lines Cr II $\lambda 6147.1$, Fe II $\lambda 6147.7$, and Fe II $\lambda 6149.2$, and Zeeman patterns of those lines. The patterns are represented in the conventional manner, by bars whose length is proportional to the relative strength of the components. The $\pi$ components appear above the horizontal (wavelength) axis; the $\sigma$ components below it. For the sake of clarity, the wavelengths in the stellar spectra have been reduced to the laboratory reference frame

Besides being a Zeeman doublet, Fe II $\lambda 6149.2$ is a particularly good diagnostic line for the determination of the mean magnetic field modulus because

1. it is sufficiently far in the red to take advantage of both the maximum sensitivity of CCD detectors and of the quadratic increase of the Zeeman effect with wavelength (the first magnetic field modulus determinations were performed using photographic plates, that is from the observation of the blue part of the stellar spectra);

2. it is observed in almost all Ap stars (like many Fe II lines);

3. the distribution of iron over the surface of Ap stars is usually rather homogeneous, so that the use of a Fe II line for magnetic field diagnosis allows a fairly uniform sampling of the stellar surface; 
4. among the red Fe II lines, Fe II $\lambda 6149.2$ is the one with the largest magnetic sensitivity;

5. this line is rather "clean" in most stars: on the red side, it is free from any strong blend in almost all the studied stars. On the blue side, it is often blended by one (or in some stars, possibly two - see e.g. the spectrum of HD 93507 shown below in Fig. 3) unidentified line(s). But in most cases this blend is relatively weak, and it can be handled adequately in the measurement procedure (see below).

The main drawback of the use of Fe II $\lambda 6149.2$ is that it is not formed in a regime of pure Zeeman effect, but rather in a regime of partial Paschen-Back effect. Indeed, the lower level of the transition responsible for it and the lower level of the transition from which Fe II $\lambda 6147.7$ originates belong to the same spectroscopic term, and their separation is not much larger than the magnetic splitting induced by fields of kilogauss order. The magnetic field induces a mixing of the lower levels of the two considered transitions, and as a result a distortion of the profiles of the split lines Fe II $\lambda 6147.7$ and Fe II $\lambda 6149.2$. These lines become asymmetric, the former with the red split components deeper than their blue counterparts, while the red component of Fe II $\lambda 6149.2$ is less deep, but broader than the blue one. This can be seen in Fig. 1 as well as in many of the plots of the spectral region of interest illustrating this paper. A detailed study of the physics of the formation of the lines Fe II $\lambda 6147.7$ and Fe II $\lambda 6149.2$ has been presented in Paper I. The most relevant result, within the present framework, is that for fields up to a few tens kG, Eq. (1) keeps giving an excellent approximation of the wavelength separation of the split components of the doublet Fe II $\lambda 6149.2$ (see also Sect. 6), even though their intensities may become very different in strong enough magnetic fields.

In Eq. (1), $\lambda_{\mathrm{r}}$ and $\lambda_{\mathrm{b}}$ are the wavelengths of the centres of gravity of the split components of Fe II $\lambda 6149.2$. In practice, these wavelengths were determined either by direct integration of the whole component profiles or by fitting a gaussian simultaneously to each of them (see Paper II for details). The latter method was preferred for lines that are not fully split, in which the magnetic components are (almost) symmetric. The direct integration works better when the splitting is large and the split components are distorted (e.g. by rotational Doppler effect). Of course, all the intermediate situations between the two extreme "ideal cases" described above are encountered in practice, and some compromise must be adopted. With the experience of the several hundreds of measurements reported here, most of which were repeated several times, we believe that we have developed the ability to choose the "best" measurement method, which has allowed us to obtain a homogeneous set of accurate data. In support of this, it may also be mentioned that, in a number of cases when both measurement techniques appeared equally suited, the results given by both of them were quite consistent. Finally, the gaussian measurement technique proved very handy to remove the contribution of the blue blend to $\mathrm{Fe}$ II $\lambda$ 6149.2: whenever possible, a multiple fit of three of four gaussians was performed: one for each of the split components of Fe II $\lambda 6149.2$, and one or two to account for the blending line(s).

It is not straightforward to estimate the measurement uncertainties. This, in fact, is best done calling to a posteriori arguments. The discussion of this point is therefore postponed to Sect. 6.

\section{Observations and reductions}

Observations for this programme have been carried out at four observatories using various combinations of telescopes, spectrographs and detectors. Table 1 gives an overview of the observing runs partly or entirely devoted to this project (totalling 222 nights). The columns in this table are, in order:

- the dates of the beginning and of the end of the run (the format is day/month/year);

- an identification number by which we refer further in this paper to the instrumental configuration used (configurations that are practically equivalent are given the same identification);

- a brief description of the configuration, that is, the observatory where the observations were conducted, and the telescope, the instrument, and the detector that were used;

- the initials of the observer(s) (all the authors performed some of the observations).

The various instrumental configurations (and the abbreviations used in Table 1 to refer to them) are described more in detail hereafter.

At the end of this section, we also give some information about the reduction procedures.

The largest fraction of the spectra were taken at the European Southern Observatory, using the Coudé Echelle Spectrograph (CES). A description of this instrument has been given by Lindgren \& Gilliotte (1989; see also the update by Mathys 1994a). We used its long camera (LC) in most runs, with as detectors a Reticon or one of the following CCDs (referred to by their internal ESO number): RCA \#9, Ford \#24, Ford \#30, Loral \#34, and Loral \#38. All these detectors have a pixel size of $15 \mu \mathrm{m}$ (for the Reticon, this is the size in the dispersion direction, while the CCDs have square pixels). For a few runs, the observations were performed with the short camera (SC) of the CES, always in combination with CCD RCA \#9.

In general, light was fed into the CES through the 3-mirror coudé train of the Coudé Auxiliary Telescope (CAT). The corresponding instrumental configurations in Table 1 are denoted CES LC or CES SC. In those configurations, the width of the spectrograph entrance slit was adjusted to achieve a resolving power of $10^{5}$ (with the 
Table 1. Observing runs and instrumental configurations

\begin{tabular}{|c|c|c|c|c|c|c|}
\hline Dates & Id. & Site & Telescope & Instrument & Detector & Observers \\
\hline $30.04-06.05 .1988$ & 1 & $\mathrm{ESO}$ & CAT & CES LC & Reticon & GM \\
\hline 20.04-26.04.1989 & 1 & $\mathrm{ESO}$ & CAT & CES LC & CCD RCA \#9 & GM \\
\hline $25.03-30.03 .1990$ & 1 & $\mathrm{ESO}$ & CAT & CES LC & CCD RCA \#9 & GM \\
\hline 01.10-08.10.1990 & 7 & $\mathrm{OHP}$ & 152 & AURELIE & Barrette Th & GM \\
\hline 01.03-11.03.1991 & 1 & $\mathrm{ESO}$ & CAT & CES LC & CCD RCA \#9 & TL \\
\hline 08.08-16.08.1991 & 7 & $\mathrm{OHP}$ & 152 & AURELIE & Barrette Th & GM, JDL, JM \\
\hline 20.11-26.11.1991 & 7 & $\mathrm{OHP}$ & 152 & AURELIE & Barrette Th & JDL \\
\hline 08.01-16.01.1992 & 7 & $\mathrm{OHP}$ & 152 & AURELIE & Barrette Th & JDL \\
\hline 19.04-20.04.1992 & 2 & $\mathrm{ESO}$ & CAT & CES SC & CCD RCA \#9 & GM \\
\hline 14.06-19.06.1992 & 1 & $\mathrm{ESO}$ & CAT & CES LC & CCD RCA $\# 9$ & GM \\
\hline 06.08-08.08.1992 & 1 & $\mathrm{ESO}$ & CAT & CES LC & CCD FA \#24 & GM \\
\hline $14.10-16.10 .1992$ & 1 & ESO & CAT & CES LC & CCD FA \#30 & GM \\
\hline 16.10-19.10.1992 & 4 & $\mathrm{ESO}$ & CAT & CES LC/F200 & CCD FA \#30 & GM \\
\hline 19.10-21.10.1992 & 5 & $\mathrm{ESO}$ & CAT & CES SC/F50 & CCD RCA \#9 & GM \\
\hline 21.10-22.10.1992 & 5 & $\mathrm{ESO}$ & CAT & CES SC/F135 & CCD RCA \#9 & GM \\
\hline $28.10-06.11 .1992$ & 7 & $\mathrm{OHP}$ & 152 & AURELIE & Barrette Th & $\mathrm{JM}$ \\
\hline 06.11-07.11.1992 & 2 & $\mathrm{ESO}$ & CAT & CES SC & CCD RCA \#9 & GM \\
\hline 06.12-08.12.1992 & 1 & ESO & CAT & CES LC & CCD FA \#30 & GM \\
\hline 02.01-04.01.1993 & 1 & $\mathrm{ESO}$ & CAT & CES LC & CCD FA \#30 & GM \\
\hline $29.01-31.01 .1993$ & 1 & ESO & CAT & CES LC & CCD FA \#30 & GM \\
\hline 31.03-02.04.1993 & 1 & ESO & CAT & CES LC & CCD FA \#30 & GM \\
\hline $22.04-24.04 .1993$ & 1 & ESO & CAT & CES LC & CCD FA \#30 & GM \\
\hline $30.04-07.05 .1993$ & 7 & $\mathrm{OHP}$ & 152 & AURELIE & Barrette Th & GM, SH \\
\hline 21.05-23.05.1993 & 1 & $\mathrm{ESO}$ & CAT & CES LC & CCD FA \#30 & GM \\
\hline 05.06-06.06.1993 & 6 & $\mathrm{ESO}$ & 3.6 & CES LC/F200 & CCD FA \#30 & GM \\
\hline $18.06-25.06 .1993$ & 9 & KPNO & Coudé feed & Coudé spectro & CCD TI \#5 & TL \\
\hline 21.06-23.06.1993 & 1 & $\mathrm{ESO}$ & CAT & CES LC & CCD FA \#30 & GM \\
\hline 21.06-28.06.1993 & 7 & $\mathrm{OHP}$ & 152 & AURELIE & Barrette Th & $\mathrm{JM}$ \\
\hline $10.08-11.08 .1993$ & 2 & $\mathrm{ESO}$ & CAT & CES SC & CCD RCA \#9 & GM \\
\hline $11.08-16.08 .1993$ & 1 & ESO & CAT & CES LC & CCD FA \#30 & GM \\
\hline $21.09-28.09 .1993$ & 9 & KPNO & Coudé feed & Coudé spectro & CCD TI \#5 & $\mathrm{TL}$ \\
\hline $20.10-26.10 .1993$ & 7 & OHP & 152 & AURELIE & Barrette Th & JM \\
\hline 03.11-10.11.1993 & 2 & $\mathrm{ESO}$ & CAT & CES SC & CCD RCA \#9 & GM \\
\hline $04.12-05.12 .1993$ & 2 & $\mathrm{ESO}$ & CAT & CES SC & CCD RCA \#9 & GM \\
\hline $13.02-15.02 .1994$ & 1 & $\mathrm{ESO}$ & CAT & CES LC & CCD FA \#30 & GM \\
\hline $17.02-26.02 .1994$ & 7 & $\mathrm{OHP}$ & 152 & AURELIE & Barrette Th & JDL \\
\hline 06.03-08.03.1994 & 1 & $\mathrm{ESO}$ & CAT & CES LC & CCD FA \#30 & GM \\
\hline 24.03-26.03.1994 & 2 & ESO & CAT & CES SC & CCD RCA \#9 & GM \\
\hline $13.04-15.04 .1994$ & 1 & $\mathrm{ESO}$ & CAT & CES LC & CCD FA \#30 & GM \\
\hline $21.05-23.05 .1994$ & 1 & ESO & CAT & CES LC & CCD FA \#30 & GM \\
\hline $11.06-12.06 .1994$ & 6 & $\mathrm{ESO}$ & 3.6 & CES LC/F200 & CCD FA \#30 & GM \\
\hline $27.06-02.07 .1994$ & 1 & ESO & CAT & CES LC & CCD FA \#30 & GM \\
\hline 15.07-20.07.1994 & 8 & $\mathrm{OHP}$ & 193 & ELODIE & CCD Tek & JM \\
\hline $24.07-27.07 .1994$ & 7 & $\mathrm{OHP}$ & 152 & AURELIE & Barrette $\mathrm{Th}$ & JM \\
\hline $10.08-17.08 .1994$ & 7 & $\mathrm{OHP}$ & 152 & AURELIE & Barrette Th & JM \\
\hline 04.09-12.09.1994 & 7 & $\mathrm{OHP}$ & 152 & AURELIE & Barrette Th & GM, SH \\
\hline 08.12-14.12.1994 & 9 & KPNO & Coudé feed & Coudé spectro & CCD TI \#5 & TL \\
\hline 22.12-24.12.1994 & 1 & $\mathrm{ESO}$ & CAT & CES LC & CCD Loral \#34 & GM \\
\hline $11.01-14.01 .1995$ & 10 & CFHT & CFHT & $\mathrm{F} / 4$ spectro & CCD Loral \#3 & JDL \\
\hline $03.02-05.02 .1995$ & 1 & $\mathrm{ESO}$ & CAT & CES LC & CCD Loral \#34 & GM \\
\hline $12.02-14.02 .1995$ & 11 & $\mathrm{ESO}$ & NTT & EMMI + R4 grt. & CCD Tek \#36 & GM \\
\hline $16.03-17.03 .1995$ & 1 & $\mathrm{ESO}$ & CAT & CES LC & CCD Loral \#34 & GM \\
\hline 23.03-25.03.1995 & 1 & $\mathrm{ESO}$ & CAT & CES LC & CCD Loral \#34 & GM \\
\hline $20.04-22.04 .1995$ & 1 & ESO & CAT & CES LC & CCD Loral \#34 & GM, SH \\
\hline 15.05-17.05.1995 & 1 & $\mathrm{ESO}$ & CAT & CES LC & CCD Loral \#34 & GM \\
\hline $12.06-14.06 .1995$ & 1 & $\mathrm{ESO}$ & CAT & CES LC & CCD Loral \#34 & GM \\
\hline 09.07-11.07.1995 & 1 & $\mathrm{ESO}$ & CAT & CES LC & CCD Loral \#34 & GM, SH \\
\hline 17.08-20.08.1995 & 3 & $\mathrm{ESO}$ & CAT & CES LC & CCD Loral \#38 & GM \\
\hline
\end{tabular}


Table 2. Ap stars with resolved magnetically split lines

\begin{tabular}{|c|c|c|c|c|c|c|c|c|}
\hline$\overline{\mathrm{HD}} / \mathrm{HDE}$ & Other id. & $V$ & Sp. type & Period & Ref. & $\mathrm{HJD}_{0}$ & Phase origin & Ref. \\
\hline 965 & $\mathrm{BD}-0^{\circ} 21$ & 8.624 & A8p Sr & $\gg 2 \mathrm{y}$ & & & & \\
\hline 2453 & $\mathrm{BD}+31^{\circ} 59$ & 6.893 & A1p SrEuCr & $521 \mathrm{~d}$ & & 2442288.000 & $\left\langle H_{\mathrm{z}}\right\rangle \min$. & 1 \\
\hline 9996 & HR 465 & 6.376 & B9p CrEuSi & $21: y$ & 2 & & & \\
\hline 12288 & $\mathrm{BD}+68^{\circ} 144$ & 7.750 & $\mathrm{~A} 2 \mathrm{p} \mathrm{CrSi}$ & $34^{\mathrm{d}} .79$ & & 2448484.200 & $\langle H\rangle \min$. & \\
\hline 14437 & $\mathrm{BD}+42^{\circ} 502$ & 7.261 & B9p CrEuSi & & & & & \\
\hline 18078 & $\mathrm{BD}+55^{\circ} 726$ & 8.265 & $\mathrm{~A} 0 \mathrm{p} \mathrm{SrCr}$ & & & & & \\
\hline 29578 & $\mathrm{CPD}-54^{\circ} 685$ & 8.495 & A5p $\mathrm{SrEuCr}$ & $>2 \mathrm{y} ?$ & & & & \\
\hline 47103 & $\mathrm{BD}+20^{\circ} 1508$ & 9.148 & Ap SrEu & & & & & \\
\hline 50169 & $\mathrm{BD}-1^{\circ} 1414$ & 8.994 & A3p $\mathrm{SrCrEu}$ & $\gg 4 \mathrm{y}$ & & & & \\
\hline 55719 & HR 2727 & 5.302 & A3p $\mathrm{SrCrEu}$ & $847 \mathrm{~d}$ or $775 \mathrm{~d} ?$ & & & & \\
\hline 59435 & $\mathrm{BD}-8^{\circ} 1937$ & 7.972 & A4p SrCrSi & $\gtrsim 1000 \mathrm{~d}$ & & & & \\
\hline 61468 & $\mathrm{CoD}-27^{\circ} 4341$ & 9.839 & A3p $\mathrm{EuCr}$ & $>120 \mathrm{~d} ?$ & & & & \\
\hline 65339 & $53 \mathrm{Cam}$ & 6.032 & A3p SrEuCr & 8. 0267 & 3 & 2435855.652 & positive crossover & 3 \\
\hline 70331 & $\mathrm{CoD}-47^{\circ} 3803$ & 8.898 & B8p Si & 3.03 or $3.65 ?$ & & & & \\
\hline 75445 & $\mathrm{CoD}-38^{\circ} 4907$ & 7.142 & $\mathrm{~A} 3 \mathrm{p} \mathrm{SrEu}$ & $<450 \mathrm{~d} ?$ & & & & \\
\hline 81009 & HR 3724 & 7.200 & A3p CrSrSi & 33.96 & 4 & 2444486.000 & $\langle H\rangle \min$. & \\
\hline 93507 & $\mathrm{CPD}-67^{\circ} 1494$ & 8.448 & $\mathrm{~A} 0 \mathrm{p} \mathrm{SiCr}$ & $556 \mathrm{~d}$ & & 2449800.000 & $\langle H\rangle \min$. & \\
\hline 94660 & HR 4263 & 6.112 & A0p EuCrSi & $\gtrsim 2700 \mathrm{~d}$ & & & & \\
\hline 110066 & HR 4816 & 6.410 & A1p $\mathrm{SrCrEu}$ & 4900: d & 5 & & & \\
\hline 116114 & $\mathrm{BD}-17^{\circ} 3829$ & 7.026 & F0p SrCrEu & $\gg 3 \mathrm{y}$ & & & & \\
\hline 116458 & HR 5049 & 5.672 & A0p SiEuCr & $147^{\mathrm{d}} \cdot 9$ & 6 & 2447600.000 & arbitrary & 6 \\
\hline 119027 & $\mathrm{CoD}-28^{\circ} 10204$ & 10.027 & A3p SrEu & & & & & \\
\hline 126515 & $\mathrm{BD}+1^{\circ} 2927$ & 7.094 & $\mathrm{~A} 2 \mathrm{p} \mathrm{CrSr}$ & 129.95 & & 2437015.000 & $\langle H\rangle \max$. & 7 \\
\hline 134214 & $\mathrm{BD}-13^{\circ} 4081$ & 7.467 & F2p SrEuCr & $4.1456 ?$ & & & & \\
\hline 137909 & $\beta \mathrm{CrB}$ & 3.900 & A9p $\mathrm{SrEuCr}$ & 18.4868 & 8 & 2434204.700 & $\left\langle H_{\mathrm{z}}\right\rangle$ pos. extr. & 8 \\
\hline 137949 & $33 \mathrm{Lib}$ & 6.659 & F0p SrEuCr & $\gtrsim 75$ y? & & & & \\
\hline 142070 & $\mathrm{BD}-0^{\circ} 3026$ & 7.966 & A0p $\mathrm{SrCrEu}$ & 3.3748 & & 2449872.600 & $\langle H\rangle \min$. & \\
\hline 144897 & $\mathrm{CoD}-40^{\circ} 10236$ & 8.600 & B8p EuCr & 48.43 & & 2449133.700 & $\langle H\rangle \min$. & \\
\hline 150562 & $\mathrm{CoD}-48^{\circ} 11127$ & 9.848 & A5p EuSi? & $>1 \mathrm{y}$ & & & & \\
\hline 318107 & $\mathrm{CoD}-32^{\circ} 13074$ & 9.355 & B8p & & & & & \\
\hline 165474 & HR $6758 \mathrm{~B}$ & 7.449 & $\mathrm{~A} 7 \mathrm{p} \mathrm{SrCrEu}$ & $2.54065 ?$ & & & & \\
\hline 166473 & $\mathrm{CoD}-37^{\circ} 12303$ & 7.953 & A5p $\mathrm{SrEuCr}$ & $\gg 3.2 \mathrm{y}$ & & & & \\
\hline 177765 & $\mathrm{CoD}-26^{\circ} 13816$ & 9.155 & A5p $\mathrm{SrEuCr}$ & $\gg 2 \mathrm{y} ?$ & & & & \\
\hline 187474 & HR 7552 & 5.321 & A0p EuCrSi & $2345 \mathrm{~d}$ & 9 & 2446766.000 & $\left\langle H_{z}\right\rangle$ pos. extr. & 10 \\
\hline 188041 & HR 7575 & 5.634 & A6p $\mathrm{SrCrEu}$ & 223.9 & 6 & 2432323.000 & $\left\langle H_{\mathrm{z}}\right\rangle \min$. & 11 \\
\hline 192678 & $\mathrm{BD}+53^{\circ} 2368$ & 7.362 & $\mathrm{~A} 2 \mathrm{p} \mathrm{Cr}$ & 6.4186 & 12 & 2449113.240 & $\langle H\rangle \max$. & 13 \\
\hline 335238 & $\mathrm{BD}+29^{\circ} 4202$ & 9.242 & $\mathrm{~A} 1 \mathrm{p} \mathrm{CrEu}$ & $44^{\mathrm{d}} 0 ?$ & & & & \\
\hline 200311 & $\mathrm{BD}+43^{\circ} 3786$ & 7.708 & B9p $\mathrm{SiCrHg}$ & 51.75 & & 2449000.000 & arbitrary & \\
\hline 201601 & $\gamma \mathrm{Equ}$ & 4.700 & A9p SrEu & $\gtrsim 70 \mathrm{y}$ & 14 & & & \\
\hline 208217 & $\mathrm{CPD}-62^{\circ} 6281$ & 7.196 & A0p $\mathrm{SrEuCr}$ & 8. 44475 & 15 & 2447000.000 & arbitrary & \\
\hline 215441 & $\mathrm{BD}+54^{\circ} 2846$ & 8.851 & B9p Si & 9.487574 & 16 & 2448733.714 & max. brightness in $B$ & 16 \\
\hline 216018 & $\mathrm{BD}-12^{\circ} 6357$ & 7.623 & A7p $\mathrm{SrCrEu}$ & $\gg 3 \mathrm{y}$ & & & & \\
\hline
\end{tabular}

References: 1: Wolff (1975); 2: Rice (1988); 3: Borra \& Landstreet (1977); 4: Waelkens (1985); 5: Adelman (1981); 6: Hensberge (1993); 7: Preston (1970); 8: Kurtz (1989); 9: Mathys (1991); 10: Landstreet (unpublished; cited by Mathys 1991); 11: Wolff (1969); 12: Leroy (1995b); 13: Wade et al. (1996a); 14: Leroy et al. (1994); 15: Manfroid \& Mathys (1996); 16: North \& Adelman (1995). 
Table 2. continued

\begin{tabular}{|c|c|c|c|c|c|c|c|c|c|}
\hline$\overline{\mathrm{HD} / \mathrm{HDE}}$ & $b-y$ & $\begin{array}{r}\langle H\rangle_{\mathrm{av}} \\
(\mathrm{kG})\end{array}$ & $q$ & $\begin{array}{r}\left\langle H_{\mathrm{z}}\right\rangle_{\min } \\
(\mathrm{kG})\end{array}$ & $\begin{array}{r}\left\langle H_{\mathrm{z}}\right\rangle_{\max } \\
(\mathrm{kG})\end{array}$ & $\begin{array}{l}\text { Discovery } \\
\text { obs. date }\end{array}$ & Ref. & Remarks & Ref. \\
\hline 965 & & 4.4 & $\geq 1.05$ & & & November 10,1993 & & & \\
\hline 2453 & 0.010 & 3.7 & 1.04 & -1.0 & -0.4 & October 1,1990 & 2 & & \\
\hline 9996 & -0.055 & 4.4 & $\geq 1.30$ & -1.2 & +0.3 & October 7, 1990 & 2 & $\mathrm{SB}$ & 13 \\
\hline 12288 & 0.034 & 8.0 & 1.13 & -1.2 & -0.2 & before 1971 & 4 & $\mathrm{SB}$ & \\
\hline 14437 & & 7.5 & 1.15 & -2.3 & -0.4 & August 13, 1991 & 3 & & \\
\hline 18078 & 0.087 & 3.7 & $\geq 1.40$ & & & October 2, 1990 & 2 & & \\
\hline 29578 & 0.173 & 2.8 & $\geq 1.05$ & & & November 7, 1993 & & SB & \\
\hline 47103 & & 17.5 & $\geq 1.00$ & & & April 8, 1995 & 5 & & \\
\hline 50169 & -0.024 & 4.7 & $\geq 1.15$ & +0.7 & +2.1 & March 8, 1991 & 2 & SB & \\
\hline 55719 & 0.003 & 6.5 & 1.04 & $+1.0:$ & $+2.0:$ & May 3, 1988 & 1 & SB2 & 14 \\
\hline 59435 & 0.285 & 3.1 & $\geq 1.80$ & & & October 15, 1992 & & SB2 & 15 \\
\hline 61468 & -0.013 & 7.2 & $\geq 1.15$ & & & December 24, 1994 & & SB & \\
\hline 65339 & 0.057 & 12.7 & -1.92 & -5.4 & +4.2 & October 17, 1967 & 6 & SB & 16 \\
\hline 70331 & & 12.4 & 1.08 & -2.8 & -2.8 & October 21,1992 & & & \\
\hline 75445 & 0.165 & 3.0 & $\geq 1.00$ & & & April 15, 1994 & & & \\
\hline 81009 & 0.094 & 8.4 & 1.23 & +0.6 & +2.0 & before 1971 & 4 & Binary & 1 \\
\hline 93507 & & 7.2 & 1.09 & +1.6 & +2.6 & December 8, 1992 & & & \\
\hline 94660 & -0.081 & 6.2 & $\geq 1.05$ & -2.0 & -2.0 & May 2,1988 & 1 & SB & \\
\hline 110066 & 0.008 & 4.1 & $\geq 1.00$ & 0.0 & 0.0 & January 14, 1992 & 3 & & \\
\hline 116114 & 0.174 & 6.0 & $\geq 1.00$ & -1.9 & -1.9 & April 20, 1992 & 3 & SB & \\
\hline 116458 & -0.030 & 4.7 & 1.00 & -2.4 & -1.4 & May 1, 1988 & 1 & SB & 17 \\
\hline 119027 & 0.257 & 3.1 & $\geq 1.00$ & & & June 7, 1993 & & roAp & 18 \\
\hline 126515 & -0.052 & 12.7 & 1.68 & -2.3 & +2.0 & February 15, 1957 & 7 & & \\
\hline 134214 & 0.216 & 3.1 & 1.04 & 0.0 & 0.0 & April 20, 1992 & 3 & roAp & 19 \\
\hline 137909 & 0.141 & 5.5 & 1.08 & -1.0 & +0.8 & April 23, 1966 & 8 & SB & 20 \\
\hline 137949 & 0.196 & 4.7 & $\geq 1.00$ & +1.0 & +1.8 & March 7, 1991 & 2 & roAp & 21 \\
\hline 142070 & 0.084 & 5.0 & 1.10 & & & March 26, 1994 & & SB & \\
\hline 144897 & 0.166 & 9.0 & 1.13 & +2.0 & +2.0 & January 30, 1993 & & & \\
\hline 150562 & 0.301 & 4.9 & $\geq 1.02$ & & & March 25, 1994 & & roAp & 22 \\
\hline 318107 & 0.015 & 14.3 & $\geq 1.15$ & +2.0 & +2.0 & August 7, 1992 & & & \\
\hline 165474 & 0.167 & 6.5 & 1.05 & 0.0 & $+0.9:$ & before 1971 & 4 & & \\
\hline 166473 & 0.208 & 7.4 & $\geq 1.30$ & & & June 15, 1992 & & roAp & 23 \\
\hline 177765 & 0.248 & 3.4 & $\geq 1.00$ & & & August 14, 1993 & & & \\
\hline 187474 & -0.063 & 5.0 & $\geq 1.20$ & -1.8 & +1.8 & October 1986 & 9 & SB & 24 \\
\hline 188041 & 0.051 & 3.7 & 1.02 & +0.4 & +1.0 & before 1971 & 4 & & \\
\hline 192678 & 0.263 & 4.7 & 1.05 & +1.3 & +1.5 & October 1,1990 & 2 & & \\
\hline 335238 & -0.005 & 9.7 & $\geq 1.45$ & -1.3 & -1.3 & before 1971 & 4 & & \\
\hline 200311 & -0.044 & 8.7 & $\geq 1.25$ & -2.5 & +2.5 & before 1973 & 10 & & \\
\hline 201601 & 0.147 & 3.8 & $\geq 1.05$ & -1.0 & +0.6 & August 16, 1971 & 11 & roAp & 25 \\
\hline 208217 & 0.102 & 7.8 & 1.25: & & & August 14, 1993 & & SB & \\
\hline 215441 & 0.054 & 33.5 & 1.10 & +10.9 & +20.5 & October 24,1959 & 12 & & \\
\hline 216018 & 0.165 & 5.6 & $\geq 1.00$ & +1.3 & +1.3 & June 18, 1992 & & SB & \\
\hline
\end{tabular}

References: 1: Mathys (1990); 2: Mathys \& Lanz (1992); 3: Mathys et al. (1993); 4: Preston (1971a); 5: Babel et al. (1995); 6: Preston (1969b); 7: Preston (1970); 8: Preston (1969c); 9: Didelon (1987); 10: Adelman (1974); 11: Scholz (1979); 12: Babcock (1960); 13: Preston \& Wolff (1970); 14: Bonsack (1976); 15: North (1994); 16: Scholz \& Lehmann (1988); 17: Dworetsky (1982); 18: Martinez et al. (1993); 19: Kreidl (1985); 20: Neubauer (1944); 21: Kurtz (1991); 22: Martinez \& Kurtz (1994); 23: Kurtz \& Martinez (1987); 24: Leeman (1964); 25: Kurtz (1983). 
Table 3. Mean magnetic field modulus: number of measurements, average value, standard deviation, rms deviation from a mathematical fit, estimated uncertainty (see text)

\begin{tabular}{|c|c|c|c|c|c|}
\hline HD/HDE & $n$ & $\begin{array}{r}\text { Average } \\
\langle H\rangle \\
(\mathrm{G})\end{array}$ & $\begin{array}{r}\text { Standard } \\
\text { deviation } \\
(\mathrm{G})\end{array}$ & $\begin{array}{r}\text { rms about } \\
\text { best fit } \\
(\mathrm{G})\end{array}$ & $\begin{array}{r}\text { Estimated } \\
\text { uncertainty } \\
(\mathrm{G})\end{array}$ \\
\hline 965 & 6 & 4386 & 91 & 30 & \\
\hline 2453 & 9 & 3737 & 60 & 22 & \\
\hline 9996 & 11 & 4831 & 389 & & 30 \\
\hline 12288 & 20 & 7879 & 345 & 122 & \\
\hline 14437 & 17 & 7665 & 401 & & 200 \\
\hline 18078 & 5 & 3835 & 412 & & 30 \\
\hline 29578 & 9 & 2782 & 59 & & 40 \\
\hline 50169 & 13 & 4790 & 199 & 29 & \\
\hline 55719 & 29 & 6501 & 114 & & 60 \\
\hline 59435 & 19 & 3234 & 650 & & \\
\hline 61468 & 4 & 7321 & 546 & & 30 \\
\hline 65339 & 16 & 12840 & 3550 & 919 & \\
\hline 70331 & 31 & 12312 & 470 & & 300 \\
\hline 75445 & 9 & 2985 & 42 & & 30 \\
\hline 81009 & 39 & 8401 & 650 & 117 & \\
\hline 93507 & 28 & 7164 & 243 & 109 & \\
\hline 94660 & 17 & 6175 & 124 & & 30 \\
\hline 110066 & 4 & 4095 & 50 & & 30 \\
\hline 116114 & 18 & 5953 & 36 & & 25 \\
\hline 116458 & 15 & 4676 & 30 & 30 & \\
\hline 119027 & 12 & 3163 & 162 & & 100 \\
\hline 126515 & 20 & 12322 & 2404 & 120 & \\
\hline 134214 & 26 & 3091 & 66 & & 40 \\
\hline 137909 & 32 & 5499 & 170 & 43 & \\
\hline 137949 & 13 & 4676 & 23 & 23 & \\
\hline 142070 & 22 & 4923 & 176 & 56 & \\
\hline 144897 & 26 & 9014 & 434 & 172 & \\
\hline 150562 & 7 & 4887 & 77 & 37 & \\
\hline 318107 & 32 & 14307 & 1065 & & 300 \\
\hline 165474 & 23 & 6523 & 139 & & 25 \\
\hline 166473 & 23 & 7649 & 731 & 81 & \\
\hline 177765 & 6 & 3413 & 19 & 19 & \\
\hline 187474 & 28 & 5317 & 370 & & 25 \\
\hline 188041 & 15 & 3663 & 37 & 28 & \\
\hline 192678 & 34 & 4668 & 110 & 79 & \\
\hline 335238 & 16 & 8706 & 1119 & & 300 \\
\hline 200311 & 28 & 8568 & 649 & 300: & \\
\hline 201601 & 21 & 3846 & 59 & 35 & \\
\hline 208217 & 31 & 7958 & 588 & & 350 \\
\hline 216018 & 18 & 5643 & 81 & & 40 \\
\hline
\end{tabular}

LC, except with CCD \#38), or of $\sim 710^{4}$ (with the SC). With CCD \#38, the resolution is limited to approximately 3 pixels due to charge diffusion within the chip itself, so that when this CCD was used with the LC, the resolving power was only $\sim 710^{4}$.

In October 1992, some observations were performed replacing the coudé train of the CAT by an optical fibre linking its prime focus to the CES. Various fibres were used: two of $200 \mu \mathrm{m}$ of diameter (corresponding to 9 .' $^{\prime} 0$ on the sky), respectively blue and red optimized (with similar response at the wavelength of our observations, $6150 \AA$ ), one of $135 \mu \mathrm{m}$ of diameter, and one of $50 \mu \mathrm{m}$ of diameter. The $200 \mu \mathrm{m}$ fibres were used in combination with the long camera of the CES, and their output was fed into the spectrograph through a Bowen-Walraven image slicer giving 11 slices. This configuration is denoted "CES LC/F200" in Table 1. The other two fibres were used together with the short camera of the CES. The light coming out of the $50 \mu \mathrm{m}$ fibre was fed directly into the spectrograph, while that emerging from the $135 \mu \mathrm{m}$ fibre first passed through a 
4-slice Bowen-Walraven image slicer. These combinations are labeled "CES SC/F50" and "CES SC/F135", respectively. The resolving power achieved when using the fibres is determined by the associated image slicer (or by the fibre itself in the case of the $50 \mu \mathrm{m}$ fibre). They are of the order of 115000 for the configuration CES LC/F200, and of approximately 75000 for the other two configurations.

On two nights, light was fed to the CES from the Cassegrain focus of the $3.6 \mathrm{~m}$ telescope through a $200 \mu \mathrm{m}$ optical fibre and an 11-slice image slicer. A description of this configuration has been given by D'Odorico et al. (1989). The resolving power obtained using it is approximately 115000 .

A few spectra were also taken at ESO using the $3.5 \mathrm{~m}$ New Technology Telescope (NTT) and the ESO Multi-Mode Instrument (EMMI; Zijlstra et al. 1996), with the R4 echelle grating \#14. This grating is described by Dekker et al. (1994). We used it in the standard EMMI configuration, with the $f / 5.2$ camera and the CCD Tektronix \#36 $\left(2048 \times 2048\right.$ pixels of $\left.24 \times 24 \mu \mathrm{m}^{2}\right)$. With the entrance slit width set to $0{ }^{\prime \prime} 8$, this configuration yields a resolving power of $\sim 80000$ over a broad wavelength range (more than $2000 \AA$ ). The wavelength coverage is determined by the grism which is used as cross-disperser. The spectra discussed in this paper were taken with grisms $\# 4$, \#5, and \#6. The ranges covered by these grisms all encompass the region around $6150 \AA$, and for the purpose of diagnosing the mean magnetic field modulus from the splitting of the line Fe II $\lambda 6149.2$, they all are equivalent. Therefore, in Table 1, we do not distinguish between them.

At the Observatoire de Haute-Provence (OHP), we mostly observed for this programme with the $1.52 \mathrm{~m}$ telescope (152) and the AURELIE spectrograph (Gillet et al. 1994). The detector was a Thomson double linear array ("barrette"), which has a pixel size of $13 \times 750 \mu \mathrm{m}^{2}$. We used the 1200 grooves $\mathrm{mm}^{-1}$ grating $\# 5$ in the second order to obtain a resolving power of $\sim 710^{4}$.

A few additional spectra were recorded at OHP with the $1.93 \mathrm{~m}$ telescope (193) and the cross-dispersed echelle spectrograph ELODIE (Baranne et al. 1996). With the fixed configuration of this instrument, and its CCD Tektronix $\left(1024 \times 1024\right.$ pixels of $\left.24 \times 24 \mu \mathrm{m}^{2}\right)$ one gets a resolving power of 45000 .

At Kitt Peak National Observatory (KPNO), we observed with the $0.9 \mathrm{~m}$ coudé feed telescope and the coudé spectrograph (Willmarth 1996). We used the cross-dispersing grism No. 770 and the echelle grating $\left(31.6\right.$ grooves $\left.\mathrm{mm}^{-1}\right)$ with Camera $5(f / 3.6)$. With a slit width of $0.3 \mathrm{~mm}$, we achieved a resolving power of $1.110^{5}$ using the CCD TI \#5 $\left(15 \times 15 \mu \mathrm{m}^{2}\right.$ pixels $)$.

Finally, a few spectra were recorded with the CanadaFrance-Hawaii Telescope (CFHT) at Mauna Kea, and the $f / 4$ coudé spectrograph (GECKO; Glaspey 1993). The appropriate order of the mosaic of four 316 grooves $\mathrm{mm}^{-1}$ echelle gratings was selected using an interference filter.
The detector was the CCD Loral \#3: with its pixel size of $15 \mu \mathrm{m}$, a resolving power of $1.210^{5}$ was obtained.

The reductions were carried out using the image processing packages MIDAS (for the ESO and OHP data) and IRAF (KPNO and CFHT). The applied procedures were mostly standard, involving the following steps: electronic bias and scattered light subtraction, division by the spectrum of a white lamp for flat fielding, spectrum extraction (for two-dimensional detectors only), normalization to the continuum (fitting a suitable function through the highest points), wavelength calibration. For the latter, whenever possible (see below) the standard MIDAS option of rebinning the spectra to a constant wavelength step was not used. Instead each pixel was assigned a wavelength, which was found slightly but significantly more accurate. This is important, because the determination of the mean magnetic field modulus relies on measurements of small wavelength differences.

When appropriate, a median filter was applied to remove cosmic ray hits. Care was taken not to introduce any degradation of the line profiles when performing this operation. A few spectra, in which the diagnostic line Fe II $\lambda 6149.2$ was badly affected by cosmic events, have been discarded from this study. It cannot be ruled out, though, that in a small number of cases, cosmic ray hits in this line may have remained unnoticed: this may plausibly explain a few outlying measurements of the magnetic field modulus.

The reductions of the spectra obtained with some instrumental configurations deserve some additional, more specific comments.

In the few spectra taken using as detector a RETICON with the ESO CES, the contribution of the RETICON dark current had to be subtracted. As described by Mathys \& Solanki (1989), this was done through linear interpolation between the masked pixels at both ends of the array. With such a one-dimensional detector, though, the contributions of scattered or parasitic background light cannot be identified, thus they cannot be properly removed. However, the resulting error should be less than $0.5 \%$ of the continuum level.

Qualitatively similar but potentially more serious limitations affect the reduction of data recorded at OHP with AURELIE. Again, the one-dimensional format of the detector does not provide any information on the scattered light within the spectrograph. However, comparison of AURELIE spectra of the bright Ap star HD 137909 with quasi-simultaneous observations of this star performed with the KPNO cross-dispersed echelle spectrograph (where background contribution can in principle be accurately removed) makes us feel confident that the contribution of scattered light to our AURELIE spectra is mostly negligible. Of more concern is the high dark current of the Thomson "barrette", which was unstable. Errors in its subtraction may reach 1 to $2 \%$ of the continuum level in long exposures of faint stars. This uncertainty, of course, 


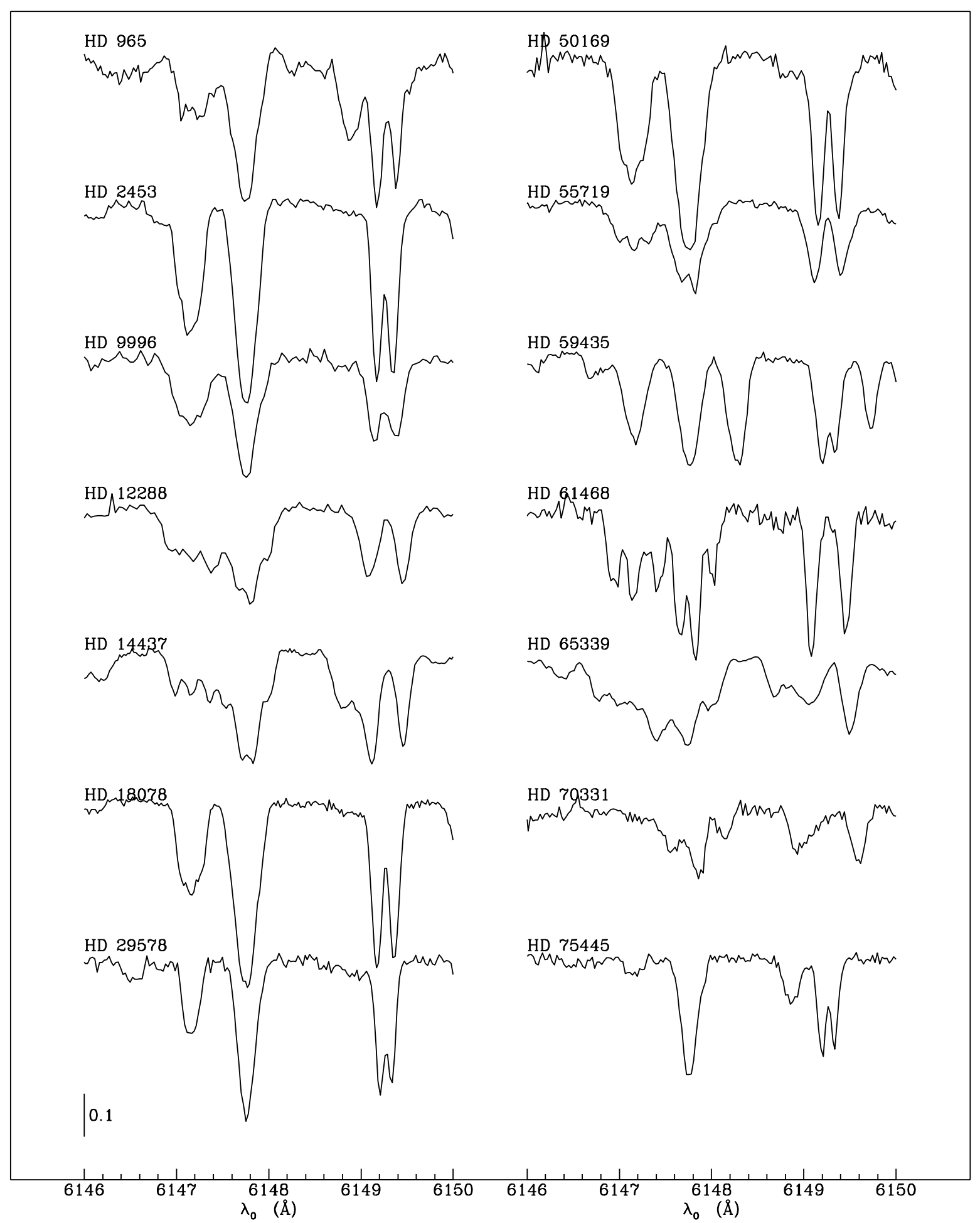

Fig. 2. Same spectral region as in Fig. 1, as observed in the stars identified next to each tracing. For the sake of clarity, the wavelengths have been reduced to the laboratory reference frame 


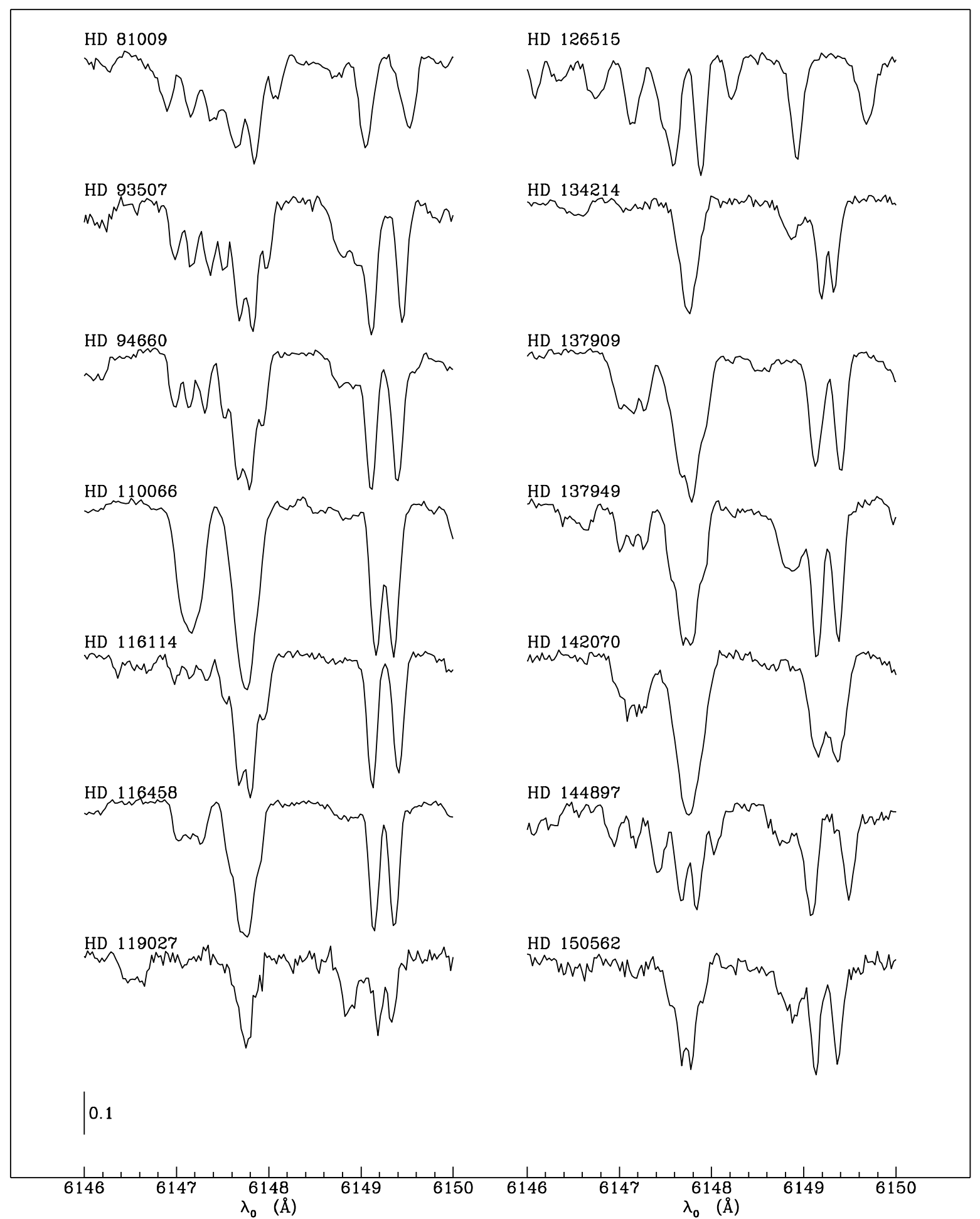

Fig. 3. Same spectral region as in Fig. 1, as observed in the stars identified next to each tracing. For the sake of clarity, the wavelengths have been reduced to the laboratory reference frame 


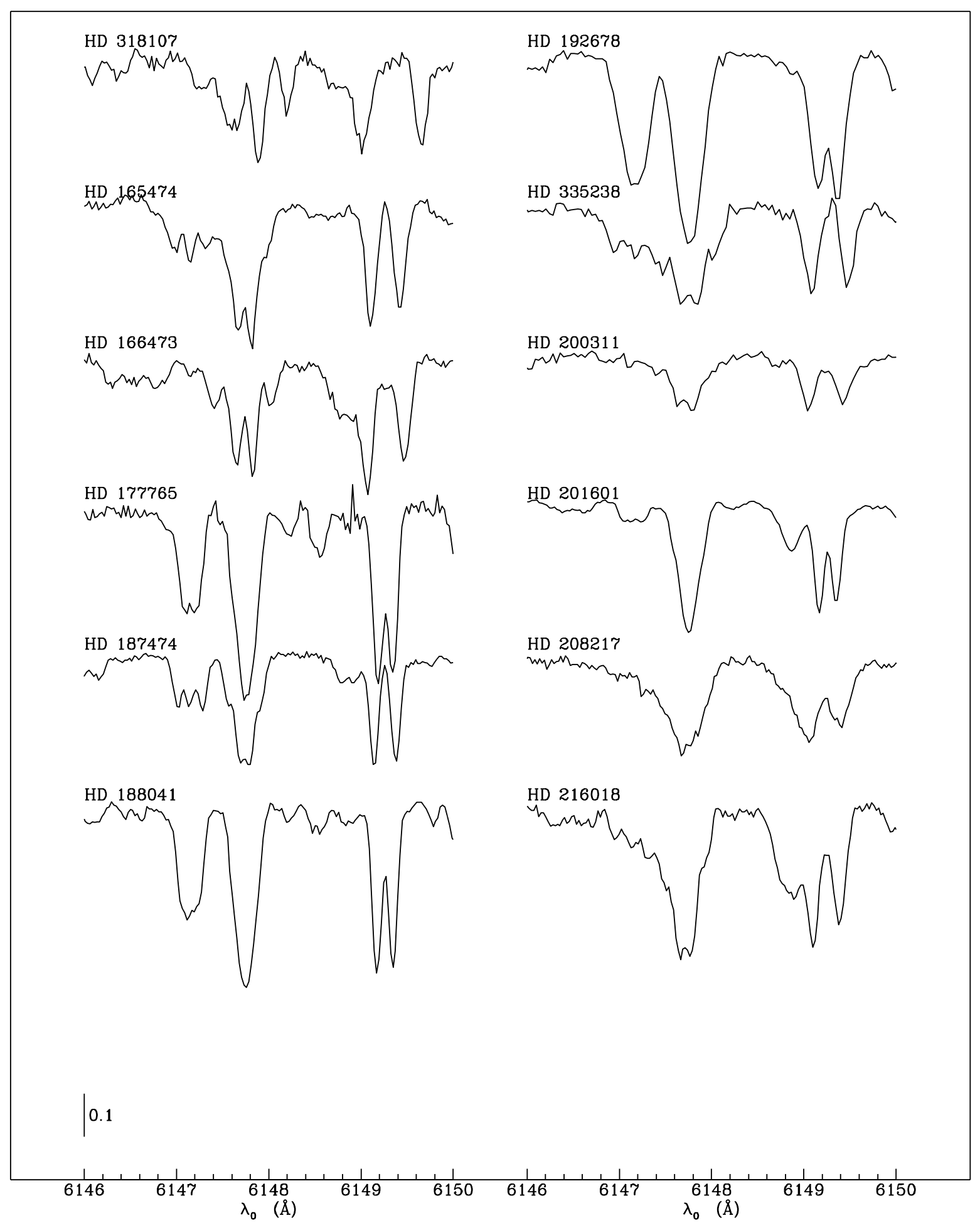

Fig. 4. Same spectral region as in Fig. 1, as observed in the stars identified next to each tracing. For the sake of clarity, the wavelengths have been reduced to the laboratory reference frame 
would affect equivalent width determinations. But it has essentially no impact on the determination of the mean magnetic field modulus.

In spectra recorded at ESO with the CES fed from the $3.6 \mathrm{~m}$ telescope or from the CAT though an optical fibre and an image slicer, the projected image of the slit on the detector within each slice is not quite perpendicular to the dispersion direction and shows some curvature. As a result, techniques developed to process long-slit spectra of extended sources must be used for the reduction. In short, the wavelength calibration is carried out individually for each row of the spectrum, and the extraction is performed only after each row has been rebinned to a constant wavelength step. Rebinning was achieved through cubic spline interpolation; the wavelength step was chosen small enough to keep the line profile degradation to a minimum.

In the CFHT spectra, the dispersion direction is along the CCD rows, and offsets of a number of columns had to be manually corrected before proceeding to the rest of the reduction, which is standard.

Not surprisingly, given the large number of spectra obtained with many different combinations of telescopes and instruments, the quality finally achieved after reduction is uneven. The vast majority of the spectra, including virtually all those recorded with the ESO CES and a large fraction of those obtained at OHP with AURELIE, have signal-to-noise $(\mathrm{S} / \mathrm{N})$ ratios between 70 and 200 . Typically, the ratio is higher for brighter stars, although there are deviations from this trend. Spectra recorded with smaller telescopes also tend to be noisier. For the faintest stars $(V=7.7)$ that could be observed with the $0.9 \mathrm{~m}$ Coudé feed at KPNO, the $S / N$ ratio in the continuum at $6149 \AA$ was limited to about 50. This results not only from the small size of the telescope, but also from the low instrumental efficiency due to the fact that the line of interest, Fe II $\lambda 6149.2$, is far from the blaze in the two orders where it can be observed (91 and 92). At ESO, with the $1.4 \mathrm{~m} \mathrm{CAT}$ and the long camera of the CES, on the best nights, $S / N=80$ was achieved in 2 hours of exposure on HD 119027, the faintest $(V=10.0)$ star with resolved lines presently known. The $1.5 \mathrm{~m}$ telescope feeding AURELIE at OHP has about the same size of the ESO CAT, but the AURELIE magnitude limit is about one magnitude lower than for the CAT + CES, due to the less good sky transparency of OHP compared to ESO and to the high readout noise of the Thomson "barrette".

\section{Results}

The purpose of our study was twofold. On the one hand, we observed repeatedly stars already known to have resolved magnetically split lines, to study the variation of their mean field modulus through their rotation cycle. On the other hand, we pursued a systematic search for additional Ap stars with magnetically resolved lines. We started by taking spectra of stars which for some reason we suspected to have resolved lines. But as time passed and as we obtained data for an increasing number of stars, we progressively widened the search, which should soon be complete for a magnitude limited sample.

We report here the discovery of 16 new stars with resolved magnetically split lines: HD 965, HD 29578, HD 59435, HD 61468, HD 70331, HD 75445, HD 93507, HD 119027, HD 142070, HD 144897, HD 150562, HDE 318107, HD 166473, HD 177765, HD 208217, and HD 216018. With the 13 stars in which the detection of magnetically resolved lines had been reported in Papers I to III, a total of 29 stars having that property have been found within the framework of the project described in this paper. Thus our work raised to 41 the number of Ap stars with resolved magnetically split lines known. Even more recently, a 42nd star of this type, HD 47103, was discovered by Babel et al. (1995).

Table 2 summarizes some properties of the 42 stars with resolved magnetically split lines known to this date. On the left page, the first four columns contain the HD/HDE number, another identification, the $V$ magnitude, and the spectral type, as it appears in the catalogue of Renson et al. (1991). The next two columns give information about the stellar rotation period and the corresponding reference. When the latter is not given, the period information in Col. 5 comes from the present paper. The heliocentric Julian date taken as origin for the computation of the rotation phases, the particular property (e.g. a magnetic extremum) characterizing the phase origin, and the relevant reference, appear in Cols. 7 to 9 . Again, the absence of a reference in Col. 9 means that the corresponding information is given in the present paper. Table 2 continues on the opposite page, where in the first column the HD/HDE number of the star is repeated, for the sake of clarity. In Col. 2, we give the Strömgren photometric index $(b-y)$, retrieved (whenever available) from Renson et al. (1991) or from Martinez (1993). Columns 3 and 4 of the right page give respectively the average $\langle H\rangle_{\text {av }}$ and the ratio $q=\langle H\rangle_{\max } /\langle H\rangle_{\min }$ of the observed maximum and minimum values of the mean magnetic field modulus. $\langle H\rangle_{\text {av }}$ is not the arithmetical average of all our measurements of the field modulus of the considered star, but rather an estimate of the mean value of $\langle H\rangle$ over the rotation cycle. When the star has been repeatedly observed throughout this cycle, $\langle H\rangle_{\text {av }}$ characterizes well its actual mean field strength, while $\langle H\rangle_{\max } /\langle H\rangle_{\min }$ is representative of the amplitude of its variations. When the phase coverage achieved so far is incomplete, these quantities only give a preliminary indication; the corresponding entries appear in italics $\left(\langle H\rangle_{\text {av }}\right)$ or as a lower limit $\left(\langle H\rangle_{\max } /\langle H\rangle_{\min }\right)$ in Table 2. The use of italics has the same meaning for the minimum $\left(\left\langle H_{z}\right\rangle_{\min }\right)$ and the maximum $\left(\left\langle H_{z}\right\rangle_{\max }\right)$ of the mean longitudinal magnetic field, which are found in Cols. 5 and 6 . The references from which the longitudinal field information has been retrieved 
Table 4. Mean magnetic field modulus

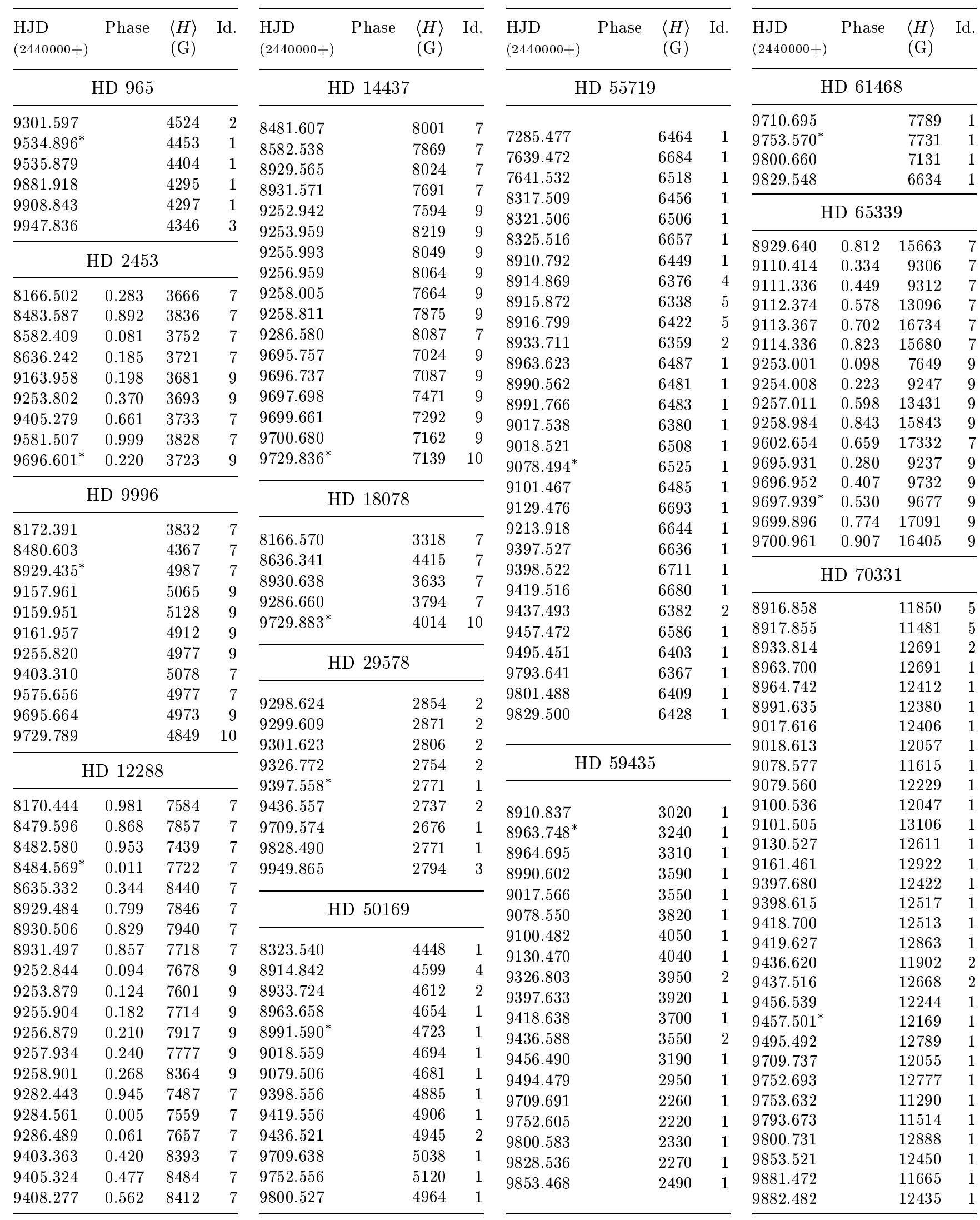


Table 4. continued

\begin{tabular}{|c|c|c|c|c|c|c|c|c|c|c|c|c|c|c|c|}
\hline $\begin{array}{l}\text { HJD } \\
(2440000+)\end{array}$ & Phase & $\begin{array}{c}\langle H\rangle \\
(\mathrm{G})\end{array}$ & Id. & $\begin{array}{l}\text { HJD } \\
(2440000+)\end{array}$ & hase & $\begin{array}{l}\langle H\rangle \\
(\mathrm{G})\end{array}$ & Id. & $\begin{array}{l}\text { HJD } \\
(2440000+)\end{array}$ & hase & $\begin{array}{l}\langle H\rangle \\
(\mathrm{G})\end{array}$ & Id. & $\begin{array}{l}\text { HJD } \\
(2440000+)\end{array}$ & hase & $\begin{array}{l}\langle H\rangle \\
(\mathrm{G})\end{array}$ & Id. \\
\hline \multicolumn{4}{|c|}{ HD 75445} & \multicolumn{4}{|c|}{ HD 93507} & \multicolumn{4}{|c|}{ HD 116114} & \multicolumn{4}{|c|}{ HD 126515} \\
\hline & & & & 8964.848 & 0.498 & 7519 & 1 & & & & & & & & \\
\hline $9457.530^{*}$ & & 2940 & 1 & 8990.769 & 0.545 & 7697 & 1 & $\begin{array}{l}8732.672 \\
8790633\end{array}$ & & & 2 & 7637.674 & 0.744 & 11896 & 1 \\
\hline 9494.528 & & 3014 & 1 & 8991.785 & 0.546 & 7448 & 1 & 8790.633 & & 59 & 1 & 7641.634 & 0.775 & 12886 & 1 \\
\hline 9495.565 & & 2961 & 1 & 9017.694 & 0.593 & 7496 & 1 & 8841.484 & & 6000 & 1 & 8321.838 & 0.009 & 15885 & 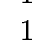 \\
\hline 9531.492 & & 3014 & 1 & 9018.750 & 0.595 & 7240 & 1 & 8990.833 & & 5933 & 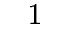 & 637.664 & 0.440 & 9931 & \\
\hline 9534.454 & & 3043 & 1 & 9078.655 & 0.703 & 7194 & 1 & 8991.815 & & 5919 & 1 & & & & 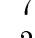 \\
\hline 9535.457 & & 2934 & 1 & 9079.612 & 0.704 & 7269 & 1 & 9017.735 & & 5937 & 1 & & & 38 & 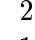 \\
\hline 9709.773 & & 3037 & 1 & 9100.613 & 0.742 & 7091 & 1 & 9018.783 & & 5933 & 1 & & & 9943 & 1 \\
\hline 9752.659 & & 2955 & 1 & 9101.581 & 0.744 & 7223 & 1 & $9079.676^{*}$ & & 5958 & 1 & 9018.809 & 0.373 & 10778 & 1 \\
\hline 9908.464 & & 2964 & 1 & 9129.545 & 0.794 & 7185 & 1 & 9101.619 & & 5917 & 1 & 9078.708 & 0.833 & 14547 & 1 \\
\hline & & & & 9130.607 & 0.796 & 6951 & 1 & 9113.435 & & 5914 & 7 & 9100.666 & 0.002 & 15705 & 1 \\
\hline \multirow{2}{*}{\multicolumn{4}{|c|}{ HD 81009}} & 9161.555 & 0.852 & 6961 & 1 & 9129.620 & & 5908 & 1 & 9110.370 & 0.077 & 16082 & 7 \\
\hline & & & & 9213.492 & 0.945 & 6862 & 1 & 9161.512 & & 5977 & 1 & 9129.589 & 0.225 & 13450 & 1 \\
\hline & & & & 9398.666 & 0.278 & 7162 & 1 & 9398.734 & & 5908 & 1 & 9145.547 & 0.348 & 11411 & 6 \\
\hline 7639.499 & 0.859 & 7875 & 1 & 9418.758 & 0.314 & 7125 & 1 & 9437.668 & & 5996 & 2 & 29 & 63 & 9799 & 1 \\
\hline 7641.562 & 0.920 & 7689 & 1 & 9436.654 & 0.347 & 7573 & & 9752.794 & & 5979 & 1 & 916 & 0.464 & 9885 & 9 \\
\hline 8318.547 & 0.855 & 7810 & 1 & 56.613 & 0.382 & 7422 & 1 & 9801.758 & & 6017 & 1 & 9162.394 & 0.477 & 9664 & 7 \\
\hline 8322.523 & 0.972 & 7548 & 1 & 9494.589 & 0.451 & 7181 & 1 & 9909.460 & & 5967 & 1 & 9166.397 & 0.508 & 9488 & 7 \\
\hline 8326.531 & 0.090 & 7605 & 1 & $\begin{array}{l}9494.009 \\
9534.525\end{array}$ & 0.523 & 7353 & 1 & 9947.513 & & 6003 & 3 & 379 & 0.588 & 9933 & 2 \\
\hline 8635.594 & 0.191 & 8104 & 7 & 9710.834 & 0.840 & 7070 & 1 & \multirow{3}{*}{\multicolumn{4}{|c|}{ HD 116458}} & 9531.528 & 0.318 & 11804 & 1 \\
\hline 8636.548 & 0.219 & 8341 & 7 & 52.761 & 0.915 & 6936 & 1 & & & & & $9752.844^{*}$ & 0.021 & 15875 & 1 \\
\hline 8789.461 & 0.721 & 8526 & 1 & 93.723 & 0.989 & 6816 & 1 & & & & & 9854.618 & 0.804 & 13387 & 1 \\
\hline 8791.455 & 0.780 & 8205 & 1 & 9800.779 & 0.001 & 6785 & 1 & & & & & & & & \\
\hline 8933.837 & 0.973 & 7573 & 2 & 9828.612 & 0.051 & 6858 & 1 & & & & 1 & \multirow{2}{*}{\multicolumn{4}{|c|}{ HD 134214}} \\
\hline 8963.782 & 0.855 & 8008 & 1 & 9853.593 & 0.096 & 6936 & 1 & & & & 1 & & & & \\
\hline 8990.735 & 0.648 & 8997 & 1 & $9881.524^{*}$ & 0.147 & 6992 & 1 & 8318.810 & 0.860 & 4639 & 1 & & & & \\
\hline 8991.678 & 0.676 & 8710 & 1 & 9908.511 & 0.195 & 7034 & 1 & 8789.620 & 0.043 & 4648 & 1 & & & & \\
\hline 9017.657 & 0.441 & 9344 & 1 & 9947.482 & 0.265 & 7217 & & 8841.520 & 0.394 & 4692 & 1 & 87 & & & 2 \\
\hline $9018.659^{*}$ & 0.471 & 9480 & 1 & & & & 3 & 9017.831 & 0.586 & 4669 & 1 & 8791.561 & & 3043 & 1 \\
\hline 9078.620 & 0.236 & 8280 & 1 & \multicolumn{4}{|c|}{ HD 94660} & 9078.685 & 0.998 & 4719 & 1 & 8842.478 & & 2970 & 1 \\
\hline 9079.649 & 0.266 & 8433 & 1 & & & & & 9100.644 & 0.146 & 4683 & 1 & 9017.807 & & 3165 & 1 \\
\hline 9100.579 & 0.883 & 7846 & 1 & 7284 & & 607 & 1 & $9130.643^{*}$ & 0.349 & 4646 & 1 & 9079.703 & & 3144 & 1 \\
\hline 9101.547 & 0.911 & 7810 & 1 & 7637.505 & & 6265 & 1 & 9160.661 & 0.552 & 4675 & 1 & 9100.693 & & 3089 & 1 \\
\hline 9129.500 & 0.734 & 8662 & 1 & 7977.746 & & 6405 & 1 & 9212.493 & 0.903 & 4721 & 1 & 9101.662 & & 3190 & 1 \\
\hline 9130.566 & 0.766 & 8584 & 1 & 8317.539 & & 6365 & 1 & 9214.593 & 0.917 & 4667 & 1 & $9129.656^{*}$ & & 3156 & 1 \\
\hline 9397.721 & 0.633 & 8842 & 1 & 8790.495 & & 6298 & 1 & 9437.652 & 0.425 & 4736 & 2 & 9130.670 & & 3156 & 1 \\
\hline 9405.435 & 0.860 & 7576 & 7 & 8963.813 & & 6260 & 1 & 9801.858 & 0.887 & 4671 & 1 & 9158.700 & & 2974 & 9 \\
\hline 9419.679 & 0.279 & 8578 & 1 & 9017.783 & & 6246 & 1 & 9854.766 & 0.245 & 4664 & 1 & 9161.698 & & 3083 & 1 \\
\hline 9437.542 & 0.805 & 7997 & 2 & 9101.637 & & 6221 & 1 & & & & & 9163.695 & & 3054 & 9 \\
\hline 9456.578 & 0.366 & 8903 & 1 & 9160.552 & & 6221 & 1 & \multirow{2}{*}{\multicolumn{4}{|c|}{ HD 119027}} & 9214.545 & & 3070 & 1 \\
\hline 9457.547 & 0.394 & 9073 & 1 & 9397.770 & & 6128 & 1 & & & & & 9398.761 & & 3159 & 1 \\
\hline 9494.550 & 0.484 & 9245 & 1 & 9437.557 & & 6139 & 2 & & & & & 9419.711 & & 3100 & 1 \\
\hline 9495.537 & 0.513 & 9161 & 1 & 9495.596 & & 6091 & 1 & & & & 6 & 9436.693 & & 3102 & 2 \\
\hline 9515.498 & 0.101 & 7827 & 6 & 9535.477 & & 6059 & 1 & 37.611 & & $\begin{array}{l}3486 \\
3272\end{array}$ & $\begin{array}{l}2 \\
2\end{array}$ & 9437.643 & & 3190 & 2 \\
\hline 9531.459 & 0.571 & 9148 & 1 & $9709.789^{*}$ & & 6065 & 1 & 57.617 & & & 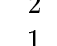 & 9456.650 & & 3108 & 1 \\
\hline 9697.021 & 0.446 & 9029 & 9 & 9801.716 & & 5927 & 1 & 9515.559 & & & 1 & 9495.758 & & 3089 & 1 \\
\hline 9698.018 & 0.475 & 9427 & 9 & 9854.498 & & 6116 & 1 & $\begin{array}{l}5.559 \\
3.785\end{array}$ & & $\begin{array}{l}3150 \\
3079\end{array}$ & 1 & 9531.560 & & 3119 & 1 \\
\hline 9699.989 & 0.533 & 9488 & 9 & 9908.481 & & 6099 & 1 & 716 & & $\begin{array}{l}3079 \\
3003\end{array}$ & 1 & 9534.619 & & 2993 & 1 \\
\hline 9701.026 & 0.564 & 9199 & 9 & \multirow{2}{*}{\multicolumn{4}{|c|}{ HD 110066}} & 91 & & $\begin{array}{l}3003 \\
315 ?\end{array}$ & & 9535.591 & & 3108 & 1 \\
\hline 9752.732 & 0.086 & 7613 & 1 & & & & & $828.681^{*}$ & & $\begin{array}{l}3152 \\
3234\end{array}$ & 11 & 9753.841 & & 2995 & 1 \\
\hline 9753.694 & 0.115 & 7825 & 1 & 8635.643 & & 4136 & 7 & 9853.667 & & $\begin{array}{l}3234 \\
3135\end{array}$ & 1 & 9829.701 & & 3026 & 1 \\
\hline 9853.557 & 0.055 & 7659 & 1 & 9110.451 & & 4140 & 7 & 9881.592 & & $\begin{array}{l}3135 \\
3343\end{array}$ & & 9882.551 & & 3026 & 1 \\
\hline 9854.462 & 0.082 & 7630 & 1 & 9157.699 & & 4060 & 9 & 9908.618 & & $\begin{array}{l}3343 \\
3098\end{array}$ & 1 & 9909.682 & & 3079 & 1 \\
\hline & & & & $9696.026^{*}$ & & 4044 & 9 & 9908.018 & & 3098 & 1 & & & & \\
\hline
\end{tabular}


Table 4. continued

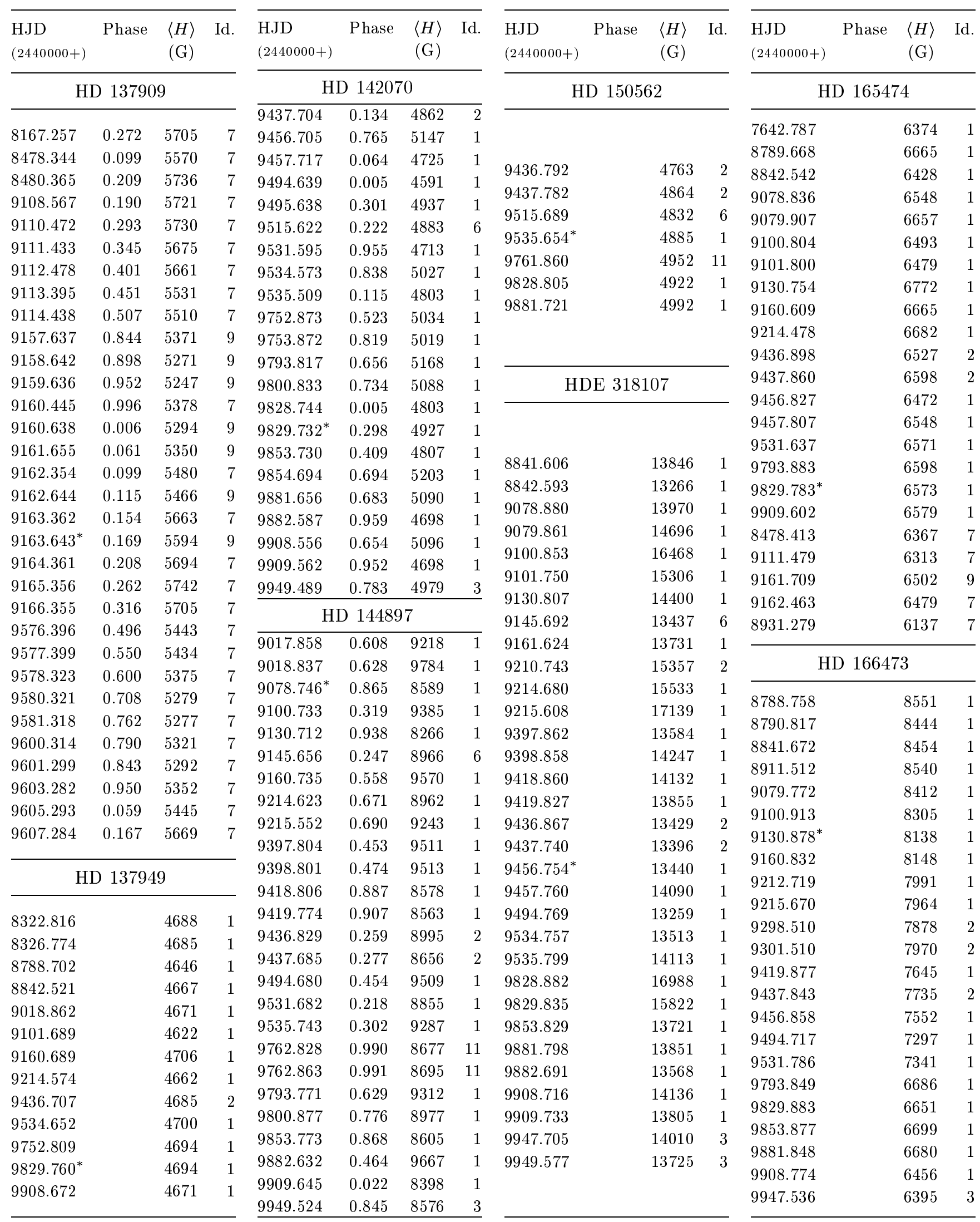


Table 4. continued

\begin{tabular}{|c|c|c|c|c|c|c|c|c|c|c|c|c|c|c|c|}
\hline & & & & & & & & & & & & & & & \\
\hline $\begin{array}{l}\text { HJD } \\
(2440000+)\end{array}$ & Phase & $\begin{array}{l}\langle H\rangle \\
(\mathrm{G})\end{array}$ & Id. & $\begin{array}{l}\text { HJD } \\
(2440000+)\end{array}$ & Phase & $\begin{array}{l}\langle H\rangle \\
(\mathrm{G})\end{array}$ & Id. & $\begin{array}{l}\text { HJD } \\
(2440000+)\end{array}$ & Phase & $\begin{array}{c}\langle H\rangle \\
(\mathrm{G})\end{array}$ & Id. & $\begin{array}{l}\text { HJD } \\
(2440000+)\end{array}$ & Phase & $\begin{array}{l}\langle H\rangle \\
(\mathrm{G})\end{array}$ & Id. \\
\hline & 17776 & & & & 1926 & & & HI & 20031 & & & II & 20 & & \\
\hline 9213.783 & & 3410 & 1 & & & & & & & & & & & & \\
\hline $9214.748^{*}$ & & 3400 & 1 & 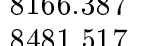 & & & 7 & 3478.523 & 0.923 & 9662 & 7 & 9213.818 & 0.153 & 8528 & 1 \\
\hline 9296.517 & & 3440 & 2 & & & & 7 & 8482.525 & 0.000 & 8935 & 7 & 9214.839 & 0.274 & 8779 & 1 \\
\hline 9300.532 & & 3425 & 2 & & & & 7 & 8484.498 & 0.039 & 9249 & 7 & 9215.873 & 0.397 & 7368 & 1 \\
\hline 9437.885 & & 3417 & 2 & חת & 0.027 & 4857 & $\begin{array}{l}7 \\
7\end{array}$ & 9110.593 & 0.137 & 8920 & 7 & 9295.577 & 0.835 & 7609 & 2 \\
\hline 9882.756 & & 3387 & 1 & 8929. & 0.356 & 4713 & 7 & 9111.600 & 0.157 & 7884 & 7 & 9296.581 & 0.954 & 7412 & 2 \\
\hline & & & & 8930 & 0.510 & 4667 & 7 & 9113.559 & 0.194 & 8018 & 7 & 9297.559 & 0.070 & 6879 & 2 \\
\hline & 18747 & & & 893 & 0.802 & 4832 & $\begin{array}{l}7 \\
7\end{array}$ & 9157.904 & 0.051 & 9316 & 9 & 9298.595 & 0.192 & 8660 & 2 \\
\hline & & & & & 574 & 47 & 7 & 9158.879 & 0.070 & 8861 & 9 & 9299.562 & 0.307 & 8354 & 2 \\
\hline 7287.859 & 0.223 & 5445 & 1 & s & 0.736 & 4818 & $\begin{array}{l}7 \\
7\end{array}$ & 9159.859 & 0.089 & 8450 & 9 & 9300.590 & 0.428 & 7307 & 2 \\
\hline 7637.845 & 0.372 & 6013 & 1 & & 0.887 & 4962 & $\begin{array}{l}7 \\
7\end{array}$ & 9160.487 & 0.101 & 8263 & 7 & 9301.549 & 0.542 & 7120 & 2 \\
\hline 8320.898 & 0.663 & 5472 & 1 & & 0.040 & 5015 & 7 & 9160.861 & 0.108 & 8645 & 9 & & & 7909 & 1 \\
\hline 8788.856 & 0.863 & 4885 & 1 & 911 & 0.198 & 4996 & 7 & 9161.876 & 0.128 & 8863 & 9 & $13^{*}$ & 0.058 & 7045 & 1 \\
\hline 8841.747 & 0.885 & 4920 & 1 & 9157 & 0.945 & 4784 & 9 & 9162.534 & 0.141 & 8668 & 7 & 949 & 0.430 & 7829 & 1 \\
\hline 8910.507 & 0.915 & 4987 & 1 & 9158.788 & 0.096 & 4828 & 9 & 9162.869 & 0.147 & 7827 & 9 & 9531.726 & 0.799 & 7605 & 1 \\
\hline 8915.491 & 0.917 & 5115 & 5 & 59.768 & 0.249 & 4564 & 9 & 9163.457 & 0.159 & 8305 & 7 & 9531.920 & 0.822 & 8268 & 1 \\
\hline 8933.523 & 0.924 & 5063 & 2 & $\begin{array}{l}0.770 \\
1792\end{array}$ & 0.405 & 4568 & 9 & 9163.868 & 0.167 & 7576 & 9 & 9534.708 & 0.152 & 8842 & 1 \\
\hline 9100.889 & 0.996 & 5013 & 1 & $\begin{array}{l}.792 \\
779\end{array}$ & 0.564 & 4614 & 9 & $9165.461^{*}$ & 0.197 & 7701 & 7 & 9534.830 & 0.166 & 8565 & 1 \\
\hline 60.943 & 0.021 & 5004 & 1 & .779 & 0.718 & 4650 & 9 & 9166.475 & 0.217 & 7682 & 7 & & & 8612 & 1 \\
\hline 9210.677 & 0.043 & 5107 & 2 & 9163.395 & 0.814 & 4904 & 7 & 9252.751 & 0.884 & 9536 & 9 & & 70 & 8419 & 1 \\
\hline 9214.805 & 0.044 & 5000 & 1 & 9163.778 & 0.874 & 4834 & 9 & 9253.723 & 0.903 & 9270 & 9 & 18 & 0.295 & 8096 & 1 \\
\hline 9296.544 & 0.079 & 5034 & 2 & 9164.430 & 0.975 & 4924 & 7 & 9255.728 & 0.942 & 8937 & 9 & 9709.534 & 0.854 & 7477 & 1 \\
\hline 9419.899* & 0.132 & 4987 & 1 & 65.393 & 0.125 & 4851 & 7 & 9256.789 & 0.962 & 8781 & 9 & 9710.569 & 77 & 7437 & 1 \\
\hline 9437.905 & 0.139 & 5004 & 2 & 6.550 & 0.306 & 4652 & 7 & 9258.724 & 0.999 & 9952 & 9 & 13 & & 8054 & 1 \\
\hline 9457.825 & 48 & 5006 & 1 & 2.664 & 0.722 & 4589 & 9 & 9281.286 & 0.435 & 7909 & 7 & & & 7961 & 1 \\
\hline 9494.811 & 0.164 & 5048 & 1 & 3.636 & 0.873 & 4820 & 9 & 9282.314 & 0.455 & 8041 & 7 & & 63 & 8987 & 1 \\
\hline 9531.752 & 0.179 & 5086 & 1 & 9254.605 & 0.024 & 4667 & 9 & 9284.407 & 0.496 & 8347 & 7 & & 30 & 7699 & 1 \\
\hline 9535.851 & 0.181 & 5059 & 1 & 9255.635 & 0.185 & 4539 & 9 & 9286.326 & 0.533 & 8303 & 7 & 09 & 0.452 & 7666 & 1 \\
\hline 9793.904 & 0.291 & 5604 & 1 & 9256.702 & 0.351 & 4612 & 9 & 9581.418 & 0.235 & 8008 & 7 & 9909.781 & 0.567 & 7257 & 1 \\
\hline 300.914 & 0.294 & 5602 & 1 & 9257.645 & 0.498 & 4643 & 9 & & & & & 9947.745 & 0.062 & 8020 & 3 \\
\hline 9828.918 & 0.306 & 5633 & 1 & 9258.637 & 0.652 & 4646 & 9 & & & & & .811 & 0.189 & 8618 & 3 \\
\hline 9853.896 & 0.317 & 5707 & 1 & 9575.601 & 0.035 & 4874 & 7 & & 20160 & & & & 0.300 & 8301 & 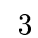 \\
\hline 9854.915 & 0.317 & 5711 & 1 & 9576.442 & 0.166 & 4641 & 7 & & & & & & & & \\
\hline 9881.868 & 0.329 & 5799 & 1 & 0.366 & 0.777 & 4740 & 7 & & & & & & & & \\
\hline 9882.916 & 0.329 & 5791 & 1 & & 0.931 & 4899 & 7 & 7637.917 & & $\begin{array}{l}3677 \\
3731\end{array}$ & $\begin{array}{l}1 \\
1\end{array}$ & & 21601 & & \\
\hline 9908.795 & 0.340 & 5835 & 1 & & & & & 8169.306 & & 3823 & 7 & & & & \\
\hline 9947.557 & 0.357 & 5952 & 3 & & E 33 & & & 8479.454 & & 3834 & 7 & 879 & & 583 & 1 \\
\hline & 8804 & & & & & & & 8925.259 & & 3893 & $\begin{array}{l}1 \\
7\end{array}$ & 8842.848 & & $\begin{array}{l}5549 \\
5692\end{array}$ & 1 \\
\hline & & & & $9163.552^{*}$ & & 8746 & 7 & 9101.916 & & 3826 & 1 & $8910.552^{*}$ & & 5621 & 1 \\
\hline & 401 & 3664 & 1 & 9165.565 & & 8832 & 7 & 9110.636 & & 3888 & 7 & 8916.617 & & 5633 & 5 \\
\hline $\begin{array}{l}8 \\
8\end{array}$ & 0.761 & 3649 & 7 & 9515.859 & & 8033 & 6 & 9160.540 & & 3840 & 7 & 8933.544 & & 5579 & 2 \\
\hline $\begin{array}{l}8324 \\
8478\end{array}$ & 0.469 & 3647 & 1 & 9549.474 & & 8026 & 8 & 771 & & 51 & 1 & 35 & & 5635 & 1 \\
\hline 8478 & 0.155 & 3691 & 7 & 9550.463 & & 8177 & 8 & 916 & & 3834 & 9 & 913 & & 5617 & 1 \\
\hline 8789 & 0.545 & 3654 & 1 & 9551.482 & & 8255 & 8 & 9215.649 & & 3842 & 1 & 9160.915 & & 5623 & 1 \\
\hline 8842 & 0.781 & 3656 & 1 & 9552.467 & & 8320 & 8 & 9257.798 & & 3842 & 9 & 9212.835 & & 5539 & 1 \\
\hline 8910 & 0.084 & 3714 & 1 & 9553.497 & & 8295 & 8 & 9281.395 & & 3930 & 7 & 9213.851 & & 5554 & 1 \\
\hline 8933 & 0.187 & 3700 & 2 & 9558.510 & & 8572 & 7 & 9456.898 & & 3867 & 1 & 9297.586 & & 5644 & 2 \\
\hline $9101.824^{*}$ & 0.939 & 3679 & 1 & 9559.490 & & 8547 & 7 & 9576.632 & & 3859 & 7 & 9301.573 & & 5608 & 2 \\
\hline 9130.850 & 0.069 & 3716 & 1 & 9560.469 & & 7825 & 7 & 969 & & 3899 & 9 & & & 5709 & 1 \\
\hline 9161.837 & 0.207 & 3645 & 1 & 9576.543 & & 11743 & 7 & $9700.578^{*}$ & & 3918 & 9 & 9710.541 & & 5621 & 1 \\
\hline 9213.690 & 0.439 & 3620 & 1 & 9603.356 & & 8406 & 7 & 9828.923 & & 3865 & 1 & 9829.908 & & 5768 & 1 \\
\hline 9256.627 & 0.630 & 3698 & 9 & 9605.357 & & 8029 & 7 & 9854.890 & & 3909 & 1 & 9882.892 & & 5791 & 1 \\
\hline 9437 & 0.440 & 3591 & 2 & 9606.363 & & 8278 & 7 & 9908.753 & & 3838 & 1 & 9908.934 & & 5814 & 1 \\
\hline 9854.807 & 0.297 & 3618 & 1 & & & & & & & & & & & & \\
\hline
\end{tabular}


are mentioned in the text. Columns 7 and 8 give the date of the first observation of magnetically resolved lines in the considered star (when known; otherwise information about the discovery year is given) and the reference of the paper where this discovery has been announced (no entry in Col. 8 means that the presence of resolved split lines is reported here for the first time). Finally, some additional remarks appear in Col. 9 (SB stands for spectroscopic binary; SB2 for double-lined spectroscopic binary; and roAp denotes a rapidly oscillating Ap star - see e.g. Kurtz 1990), with the corresponding reference in Col. 10 (if it is not the present paper).

The properties summarized in Table 2 are discussed in more detail in the rest of this paper (especially in the next section).

From the observations described in Sect. 3, we have repeatedly measured the mean magnetic field modulus of 40 of the 42 stars with magnetically resolved lines, inferring it from the wavelength shift between the components of the line Fe II $\lambda 6149.2$, as explained in Sect. 2. A portion of one spectrum of each of these 40 stars, comprising the line Fe II $\lambda 6149.2$, is shown in Figs. 2 to 4 . The two stars appearing in Table 2 for which we present no results here are HD 47103 and HD 215441. The discovery (Babel et al. 1995) of resolved magnetically split lines in the former is too recent (let us recall that this report is limited to data acquired before end of August 1995). A number of spectra of HD 215441 have been recorded in the course of this programme, but its magnetic field is so strong ( $34 \mathrm{kG}$ ) that one can no longer use Eq. (1) to interpret the splitting of Fe II $\lambda$ 6149.2: the latter must be modeled accounting properly for the physics of the partial Paschen-Back effect. This will be the subject of a future publication.

We present an overview of the measurements of the mean magnetic field modulus of the 40 remaining stars in Table 3. The columns give, in order, the HD or HDE number of the star, the number $n$ of measurements of its magnetic field modulus that we have performed, the average and the standard deviation of these measurements, their rms deviation about a best fit curve of their variations, and their estimated uncertainty. The data appearing in the last two columns are explained more in detail in Sect. 6. Note that in contrast with Table 2, the quantity given in Col. 3 of Table 3 is the plain arithmetic average of all our measurements of the considered star. The standard deviation about this average, which appears in Col. 4, results from the superposition of the measurement uncertainties and of the variations of the stellar field.

The 752 individual measurements of the mean magnetic field modulus are presented in Table 4. Column 1 contains the heliocentric Julian date of mid-observation. An asterisk next to this entry identifies the spectrum of each star that is shown in Figs. 2 to 4. In Col. 2, the stellar rotation phase is computed (whenever possible) using the ephemeris elements appearing in Cols. 5 and 7 of Table 2. The values of the mean magnetic field modulus
Table 5. Correspondence between the instrumental configuration code numbers (see Col. 2 of Table 1) and the symbols used in the plots

\begin{tabular}{rll}
\hline Id. & Configuration & Symbol \\
\hline 1 & CAT + CES LC & filled circle \\
2 & CAT + CES SC & open circle \\
3 & CAT + CES LC + CCD \#38 & asterisk \\
4 & CAT + CES LC /F200 & filled hexagon \\
5 & CAT + CES SC /fibre & open hexagon \\
6 & $3.6+$ CES LC $/$ F200 & star \\
7 & AURELIE & open square \\
8 & ELODIE & cross \\
9 & KPNO coudé feed & filled square \\
10 & CFHT F/4 spectro & filled triangle \\
11 & EMMI & open triangle \\
\hline
\end{tabular}

$\langle H\rangle$ are given in Col. 3 of Table 4, with in Col. 4 the code referring to the instrumental configuration used to perform the corresponding observation (as defined in Col. 2 of Table 1). All the mean field modulus measurements that we have performed before end of August 1995 are included in Table 4. A few of them correspond to observations that have already been presented in Papers I to III. However, for the sake of homogeneity and completeness, these spectra have been remeasured together with all the others discussed here: the revised values of the field that appear in this paper supersede the ones that had been previously published. Note that the Julian dates of some of the observations reported in Paper II were erroneous: they have been corrected in the present paper.

In the next section, we discuss the mean field modulus measurements star by star. We present plots of these measurements against rotation phase or against Julian date. In these plots, different symbols are used to distinguish measurements performed with different instrumental configurations. Unless indicated otherwise in the figure caption, the meaning of these symbols is given in Table 5 . Column 1 contains the identification number of the configuration (from Col. 2 of Table 1), of which a short reminder is given in Col. 2 (which should be self-explantory from the consideration of Table 1). The description of the corresponding plot symbol appears in Col. 3 .

\section{Notes on individual stars}

\subsection{HD 965}

HD 965 has never been studied in detail before. Since magnetically resolved lines have been discovered in this star, in November 1993, its mean magnetic field modulus seems to have been monotonically decreasing (Fig. 5). It appears highly probable that the rotation period of HD 965 is much longer than 2 years, although given the 


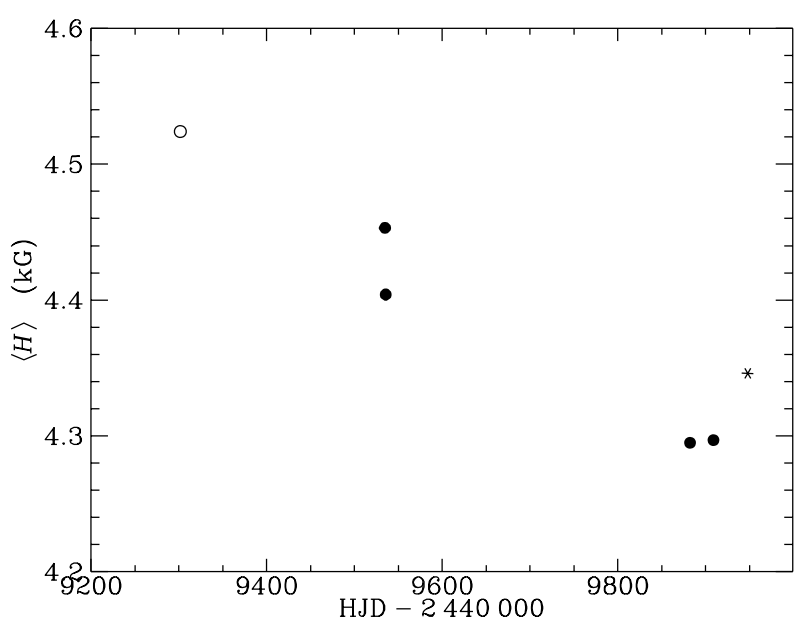

Fig. 5. Mean magnetic field modulus of HD 965 against heliocentric Julian date. The meaning of the symbols is as given in Table 5

small number of measurements, one cannot definitely rule out a shorter period.

\section{2. $H D 2453$}

The observation of resolved magnetically split lines in this star was first reported in Paper II. The only value of the period that adequately accounts for all the longitudinal field measurements (Babcock 1958; Wolff 1975; Mathys \& Hubrig 1996) is $P=(521 \pm 2) \mathrm{d}$, in good agreement with Wolff's (1975) original estimate of $525 \mathrm{~d}$. The longitudinal field is always negative, varying approximately between -950 and $-400 \mathrm{G}$.

The profile of Fe II $\lambda 6149.2$ in HD 2453 is very clean, mostly free from blends, so that we expect this star to be one of those where we can measure the magnetic field modulus with the best accuracy. As discussed in Sect. 6, the measurement uncertainty should then be of the order of $30 \mathrm{G}$. The standard deviation of our 9 measurements, 60 G (see Table 3), significantly exceeds this value, which indicates that we are likely detecting actual field variations. As a matter of fact, when plotted against rotation phase, our data seem to lie along a nearly sinusoidal curve (see Fig. 6), suggesting that the field modulus of HD 2453 may vary with a peak-to-peak amplitude of the order of $160 \mathrm{G}$. The maximum of the mean field modulus variations seems to coincide roughly with the largest negative value of the longitudinal field, and the minimum of the mean field modulus apparently occurs approximately when the longitudinal field is closest to 0 (compare Fig. 6 with Fig. 7). Additional measurements, allowing a better sampling of the rotation phases of HD 2453, will be useful to confirm that this description of the variations of the field is indeed correct.

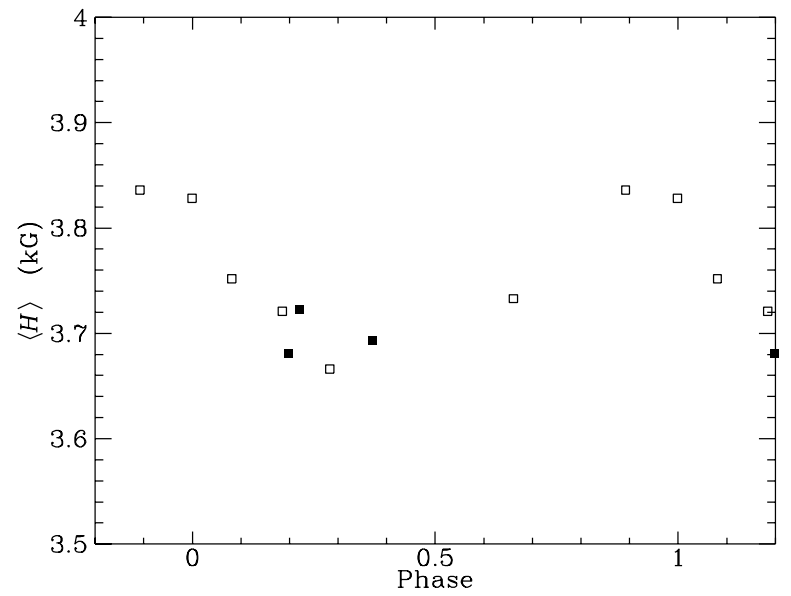

Fig. 6. Mean magnetic field modulus of HD 2453 against rotation phase. The meaning of the symbols is as given in Table 5

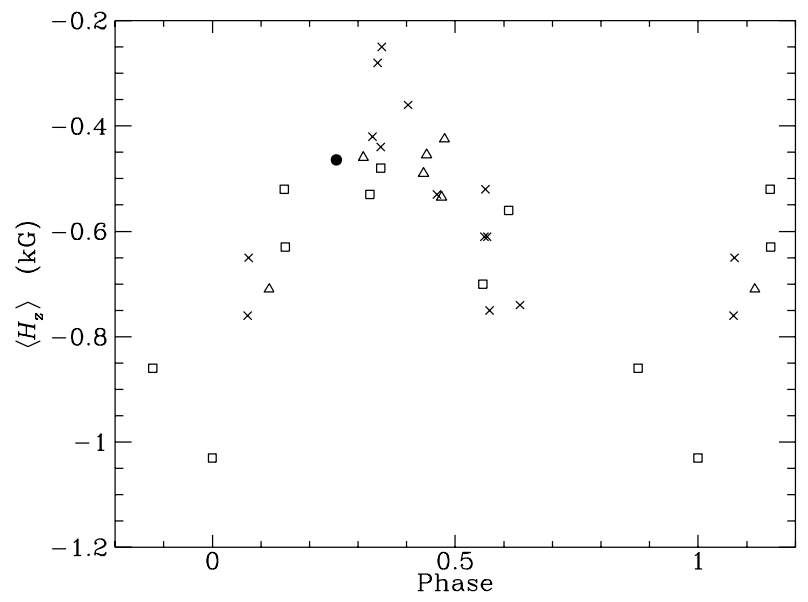

Fig. 7. Mean longitudinal magnetic field of HD 2453 against rotation phase. Data are from Babcock (1958; open triangles), Wolff $(1975$; crosses: Lick data; open squares: Mauna Kea data), and Mathys \& Hubrig (1996; filled circle)

\section{3. $H D 9996$}

In Paper II, we had reported the first observation of resolved magnetically split lines in HD 9996. From the discovery spectrum, we had measured a mean field modulus of $3.8 \mathrm{kG}$. This is significantly larger than the estimate of $2.2 \mathrm{kG}$ (Preston 1971a) and the upper limit of $2.5 \mathrm{kG}$ (Scholz 1983) obtained from the analysis of the differential broadening of unsplit lines. The resulting suspicion that the magnetic field modulus of this star may show large variations is fully confirmed by our new measurements. From the consideration of Fig. 8, where our data are plotted against Julian date, it seems that HD 9996 may just have passed the time of maximum of its mean field modulus. The latter probably occurred at the end of 


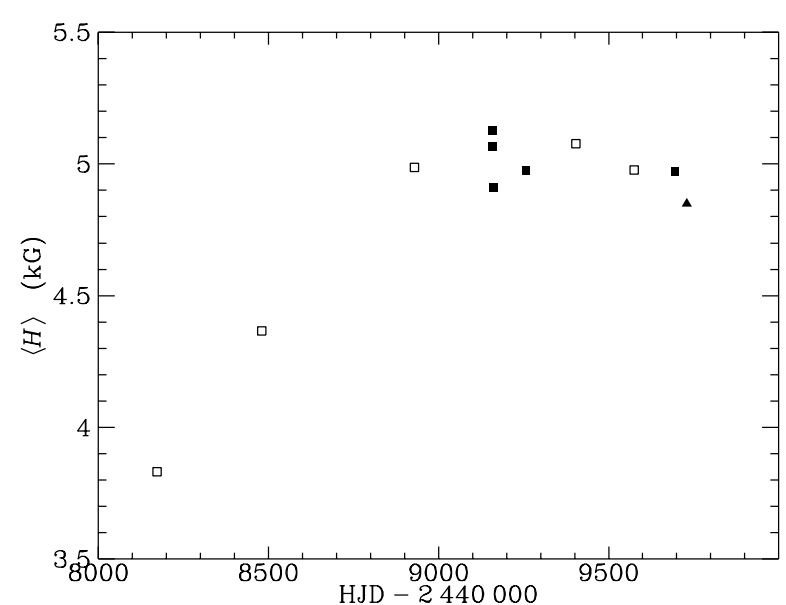

Fig. 8. Mean magnetic field modulus of HD 9996 against heliocentric Julian date. The meaning of the symbols is as given in Table 5

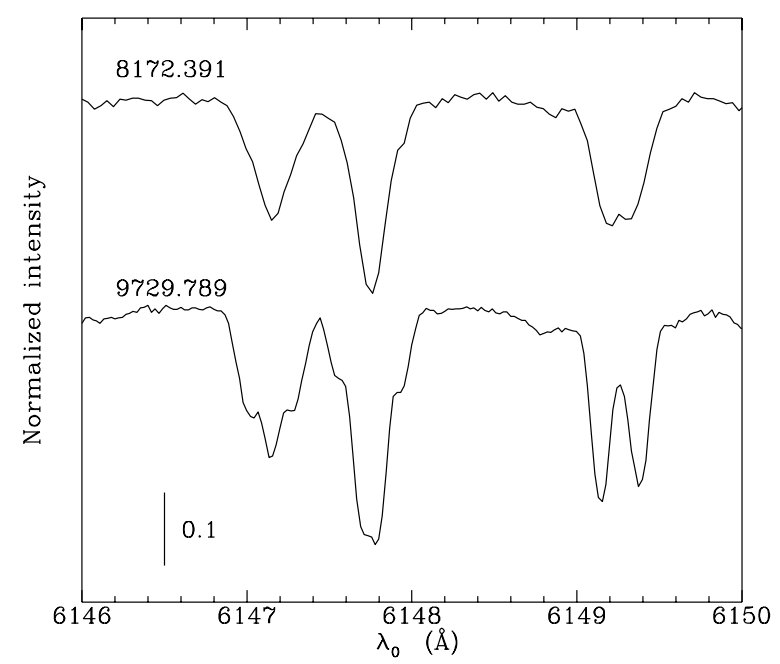

Fig. 9. Same spectral region as in Fig. 1, as observed in HD 9996 on the dates indicated next to each tracing (HJD - 2440000)

1993 or the beginning of 1994 . Adopting for the rotation period the most recent estimate of $21 \mathrm{yr}$ (Rice 1988), the phase of the maximum field modulus would appear to coincide roughly with that of the negative extremum of the mean longitudinal (or effective) field, to the accuracy with which the latter can be inferred from the published measurements (Babcock 1958; Preston \& Wolff 1970; Scholz 1978, 1983). According to the latter, the range of variation of the longitudinal field is from $-1200 \mathrm{G}$ to $+300 \mathrm{G}$.

HD 9996 was already famous for its extreme spectroscopic variations (Preston \& Wolff 1970). The results presented here indicate that the variation of its magnetic field modulus also is unusually large. Indeed, we find that the

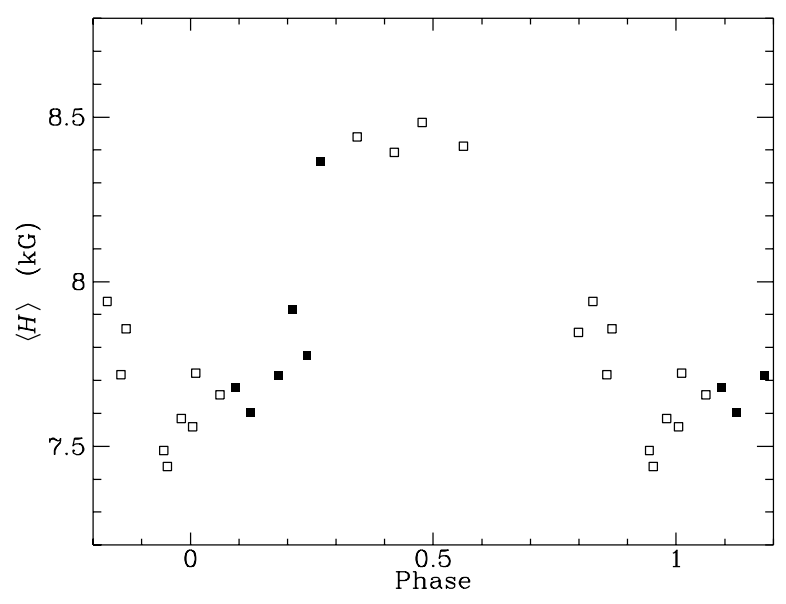

Fig. 10. Mean magnetic field modulus of HD 12288 against rotation phase. The meaning of the symbols is as given in Table 5

field reaches $5 \mathrm{kG}$ (or more) at maximum, while from the above-mentioned estimates of Preston (1971a) and Scholz (1983), at minimum it does not exceed $2.5 \mathrm{kG}$. Thus the ratio between its extrema is at least 2.0, which is greater than in any other star with magnetically resolved lines studied until now.

From the consideration of the line profiles at different phases, it furthermore appears that not only the field strength but also its orientation with respect to the observer vary remarkably with phase. This is illustrated in Fig. 9, where portions of our oldest and of our most recent spectra of the star are plotted. The very unusual, triangular shape of the line $\mathrm{Cr}$ II $\lambda 6147.1$ in the former had already been stressed in Paper II. Comparing the two observations shown in Fig. 9, one can see that this triangular shape can to a large extent be attributed to the fact that the $\sigma$ components of the line are remarkably weak. The contribution of these components to the line is seen to have increased quite noticeably between our first and last observations. Even in the latter, the strength of the $\sigma$ components relative to that of the central $\pi$ components remains smaller than in most other stars studied (see Figs. 2 to 4). Similarly, the outermost $\sigma$ components of the quadruplet Fe II $\lambda 6147.7$ are weak too, even almost not visible at all in our first spectrum. This seems to indicate that the magnetic field of HD 9996 at the considered phases is predominantly transversal, at least on the regions of the stellar surfaces where this field (which appears to be unusually inhomogeneous) is strongest.

Note also that HD 9996 is a spectroscopic binary with an orbital period $(273 \mathrm{~d})$ much shorter than its rotation period (Preston \& Wolff 1970). 


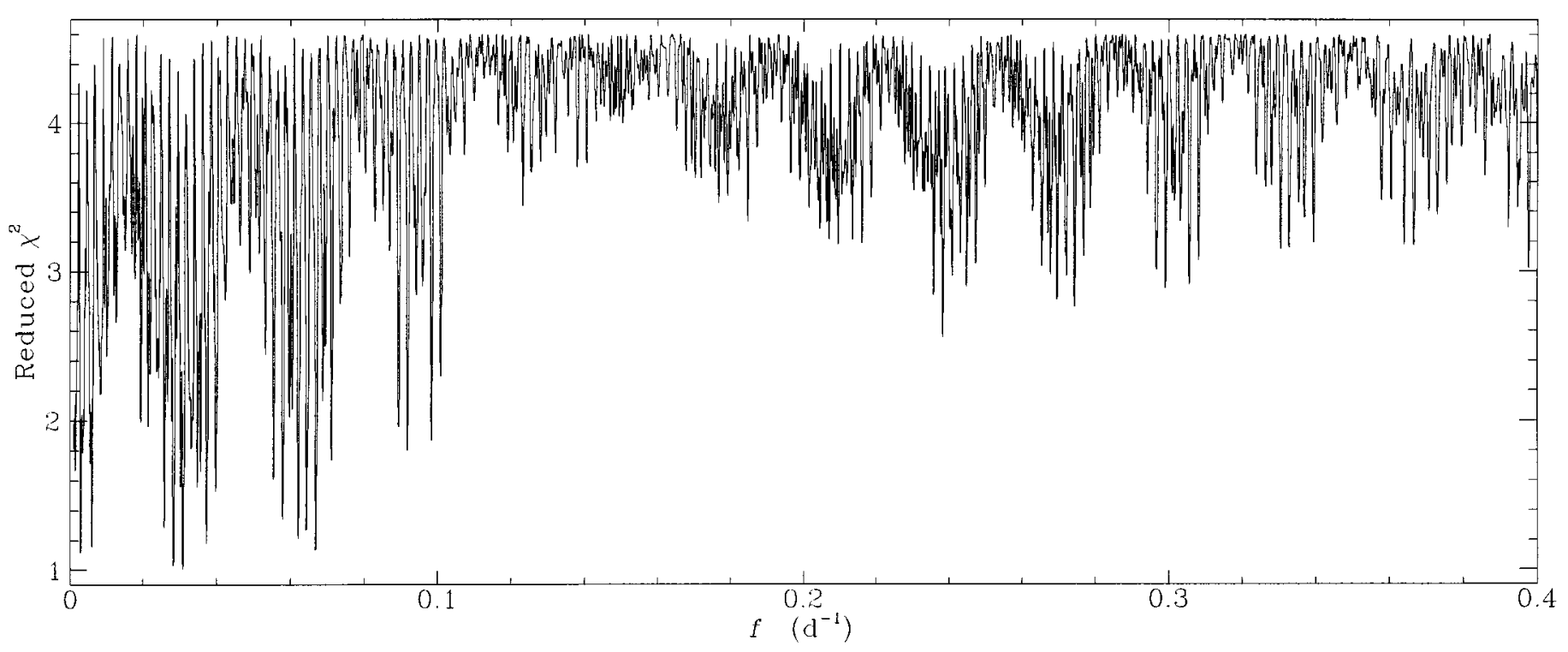

Fig. 11. Periodogram obtained in a frequency search on the measurements of the mean magnetic field modulus of HD 14437. The ordinate is the reduced $\chi^{2}$ of the fit of the field modulus measurements by a sine wave with the frequency given in abscissa

\section{4. $H D 12288$}

Analysing our 20 measurements of the mean field modulus of this star, where magnetic line splitting had first been observed by Preston (1971a), we find that the most probable value of its rotation period is

$P=(34.79 \pm 0.12) \mathrm{d}$.

This is in excellent agreement with the value $(34.9 \pm 0.3) \mathrm{d}$ that had been derived by Wolff \& Morrison (1973) from photometric observations. These photometric observations also allow us to rule out an alternative value of the period, 28.6 , which appears almost as satisfactory from the consideration of our magnetic data alone. Conversely, our measurements are inconsistent with the aliases close to 1 day that appeared marginally plausible from the photometry.

The variation of the mean magnetic field modulus of HD 12288 through its rotation cycle is shown in Fig. 10. The amplitude of the variations, of the order of $1 \mathrm{kG}$ peakto-peak, is much smaller than the difference between the extreme values of 6.1 and $8.6 \mathrm{kG}$ of the field modulus reported by Preston (1971a). However, this discrepancy, the origin of which is unclear, does not cast doubt on the reliability of the rotation period derived here.

The split components of the line Fe II $\lambda 6149.2$ show some evidence of distortion due to rotational Doppler effect. This is well seen in the portion of the spectrum shown in Fig. 2, from the fact that the red component of the line is sharper and narrower than the blue component. This is the opposite of the characteristic asymmetry corresponding to the partial Paschen-Back effect regime of formation of this line, which is actually observed when rotation is negligible (see Paper I for details).

We have obtained three measurements of the longitudinal magnetic field of HD 12288 through $\mathrm{H} \beta$ photopolarimetry: two null values at phases 0.875 and 0.974 , and a marginal detection $(-1.2 \pm 0.5 \mathrm{kG})$ at phase 0.129 .

Finally, our study reveals that the radial velocity of HD 12288 is variable, thus that it is a spectroscopic binary. The amplitude of the variation is at least $16 \mathrm{~km} \mathrm{~s}^{-1}$. The timescale on which it occurs is long: the orbital period is unlikely to be much shorter than 4 years. The determination of the orbital parameters, and more generally, the detailed study of the radial velocity of this and of the other stars considered in this paper are beyond its scope. They will be the subject of a separate work.

\section{5. $H D 14437$}

The detection of resolved magnetically split lines in HD 14437 has been reported in Paper III. Its mean field modulus appears to vary with an amplitude of the order of $1 \mathrm{kG}$ peak-to-peak. The period cannot be unambiguously determined from our present data. In a periodogram covering the range comprised between 2.5 and $1000 \mathrm{~d}$ (Fig. 11), three groups of periods clearly stand out, around $15 \mathrm{~d}$, $30 \mathrm{~d}$, and $350 \mathrm{~d}$. The latter seems to be ruled out by the fact that we observe apparently significant variations on timescales of a few days. The longitudinal field is also reported by Glagolevskij et al. (1982) and Glagolevskij et al. (1986) to vary on similarly short timescales between -2.3 and $-0.4 \mathrm{kG}$. This is consistent with our own determinations of the longitudinal field (3 measurements over one 


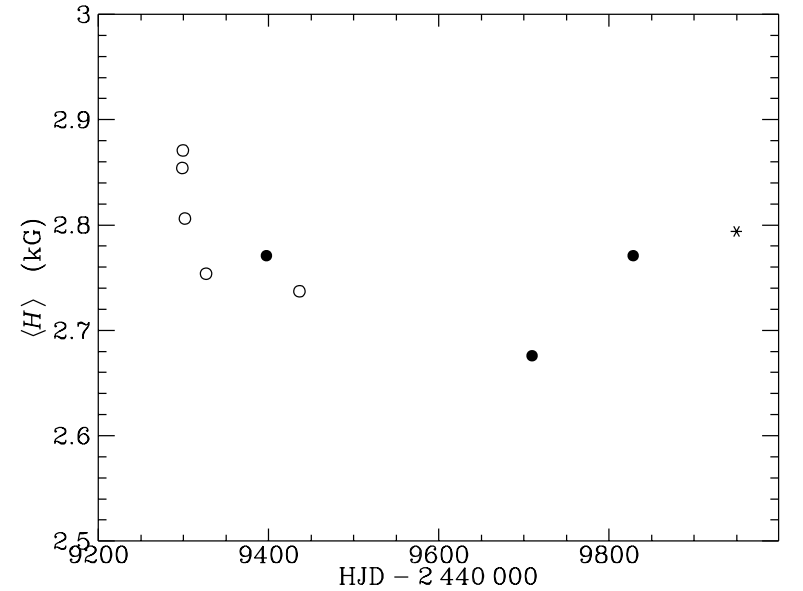

Fig. 12. Mean magnetic field modulus of HD 29578 against heliocentric Julian date. The meaning of the symbols is as given in Table 5

week, through $\mathrm{H} \beta$ photopolarimetry, ranging between -1.4 and $-1.9 \mathrm{kG})$. We do not have enough data yet to decide between the two groups of periods around $15 \mathrm{~d}$ and around $30 \mathrm{~d}$, nor to resolve the strong aliasing within these groups.

\section{6. $H D 18078$}

In the first spectrum (taken in October 1990) of HD 18078 where we observed magnetic splitting in Fe II $\lambda 6149.2$ (Paper II), this doublet was just barely resolved. Its components were better separated in January 1992. But they were hardly resolved in November 1992 and October 1993: the field modulus values derived on these dates are rather uncertain. In February 1994, splitting was not seen, but the total width of the line was still consistent with a field of the order of $3 \mathrm{kG}$. The doublet was again resolved in our last observation of this star, in January 1995. From these observations, the mean magnetic field modulus of HD 18078 appears to undergo quite significant variations (the ratio between the extrema is at least 1.4). The split components of Fe II $\lambda 6149.2$ are unusually broad. Whether this is due to rotational Doppler effect or to a particularly inhomogeneous magnetic field distribution on the stellar surface is at present unclear. Indeed, the distribution in time of our observations, which was inspired by Wolff \& Morrison's (1973) statement that the rotation period might exceed 10 years, does not allow us to decide whether the period is indeed very long or whether it might be short enough so that rotational Doppler effect could significantly contribute to the line profiles. Our future observations will be planned so as to resolve this ambiguity.

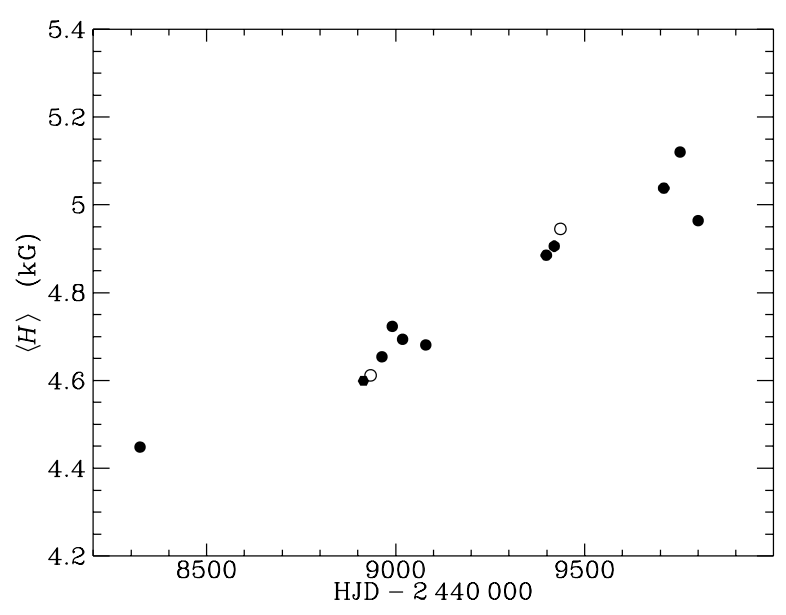

Fig. 13. Mean magnetic field modulus of HD 50169 against heliocentric Julian date. The meaning of the symbols is as given in Table 5

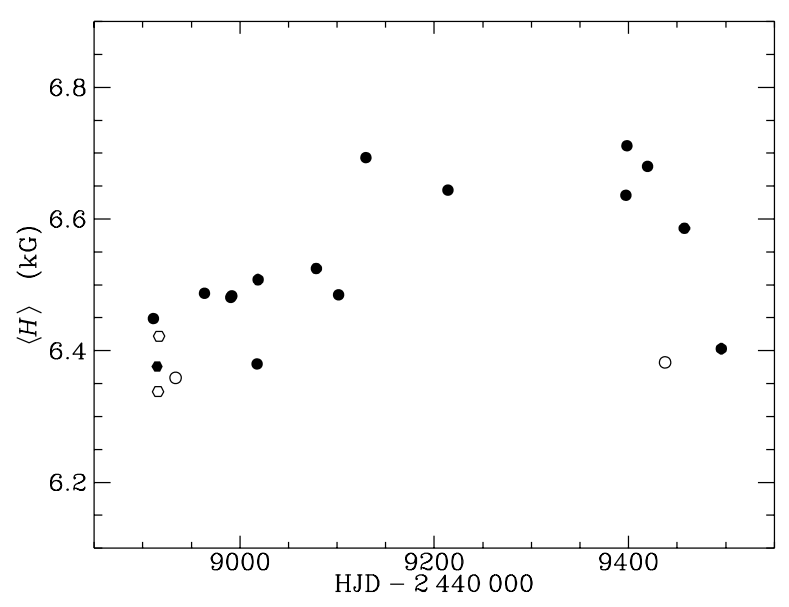

Fig. 14. Mean magnetic field modulus of HD 55719 against heliocentric Julian date (a time span of 700 days has been extracted from the whole dataset). The meaning of the symbols is as given in Table 5

\section{7. $H D 29578$}

The observations of magnetically resolved lines in this otherwise not well known Ap star is reported here for the first time. The standard deviation of $59 \mathrm{G}$ of our 9 measurements of the mean magnetic field modulus of a star where the purity of the Fe II $\lambda 6149.2$ profile probably allows us to determine this quantity with a significantly better accuracy (see Sect. 6) indicates that we are almost certainly detecting low-amplitude variations. A plot of the measurements against Julian dates (Fig. 12) suggests that the period may be long (significantly exceeding 2 years), but more observations are required to establish this more reliably. 


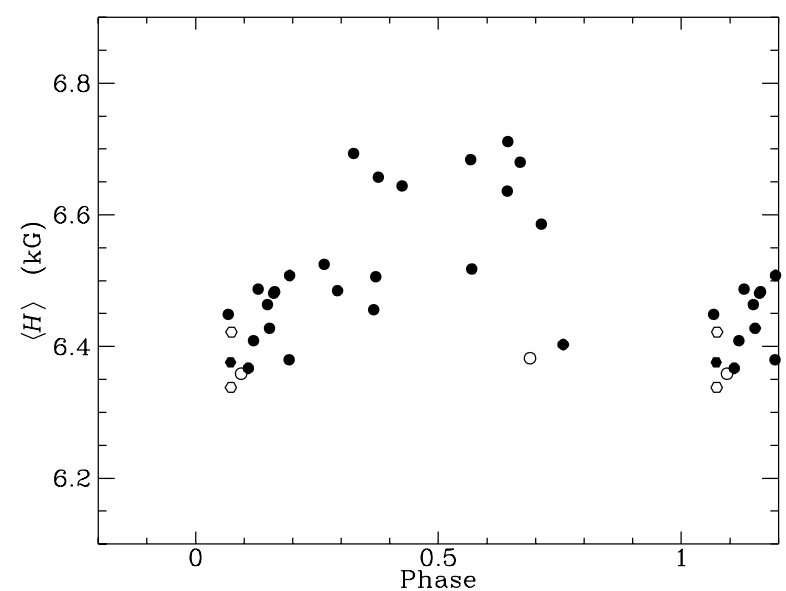

Fig. 15. Mean magnetic field modulus of HD 55719 against rotation phase, computed assuming that the rotation period is $847 \mathrm{~d}$. The phase origin is HJD 2447160.0. The meaning of the symbols is as given in Table 5

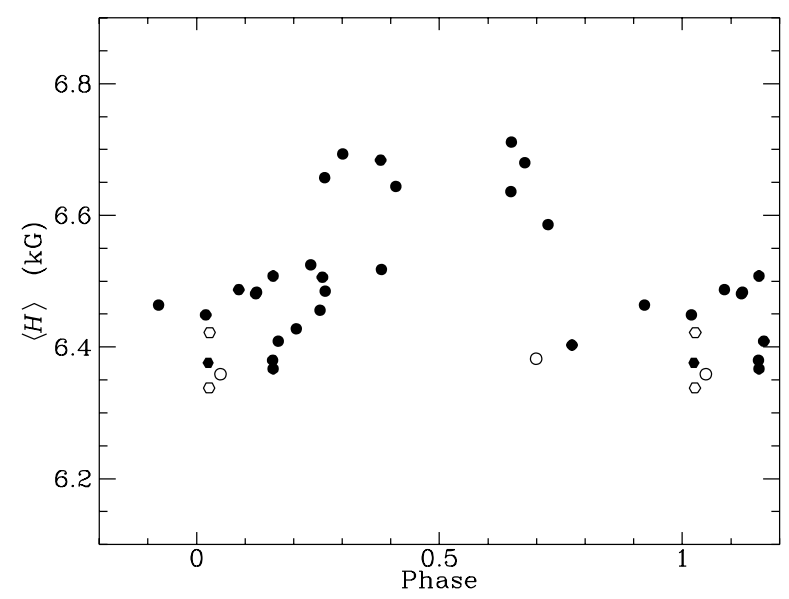

Fig. 16. Mean magnetic field modulus of HD 55719 against rotation phase, computed assuming that the rotation period is 775 d. The phase origin is HJD 2447346.0. The meaning of the symbols is as given in Table 5

On the other hand, the radial velocity of HD 29578 is definitely slowly variable. A maximum has been reached beginning of 1994, which differs by more than $16 \mathrm{~km} \mathrm{~s}^{-1}$ from the lowest value recorded so far, in our last observation of the star, in August 1995: HD 29578 appears to be a spectroscopic binary with an orbital period significantly longer than 2 years.

\subsection{HD 50169}

Since our discovery of resolved magnetically split lines in this star in March 1991 (Paper II) until our second last observation in February 1995, its mean field modulus has

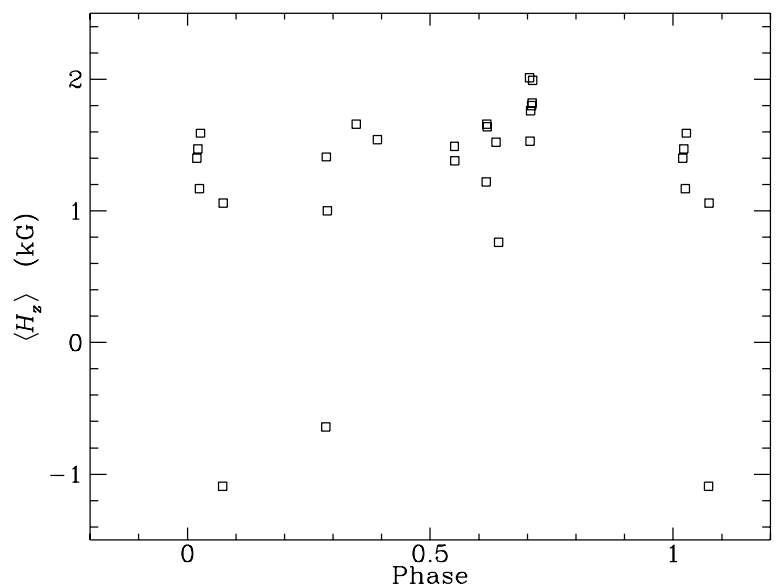

Fig. 17. Mean longitudinal magnetic field of HD 55719 against rotation phase, computed assuming that the rotation period is $847 \mathrm{~d}$ (data from Bonsack 1976). The phase origin is HJD 2447160.0

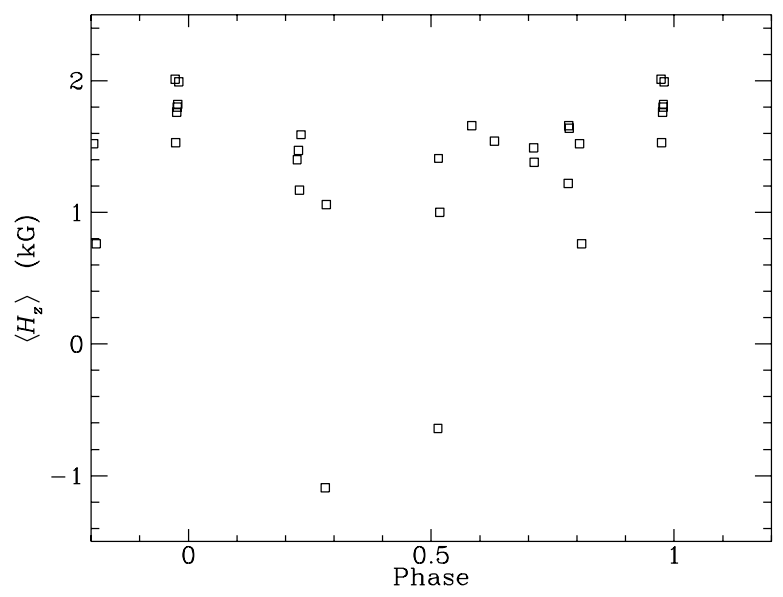

Fig. 18. Mean longitudinal magnetic field of HD 55719 against rotation phase, computed assuming that the rotation period is $775 \mathrm{~d}$ (data from Bonsack 1976). The phase origin is HJD 2447346.0

monotonically increased from 4.4 to $5.1 \mathrm{kG}$ (Fig. 13). Our last observation in March 1995 yielded a somewhat smaller field value (just below $5 \mathrm{kG}$ ), which most likely is spurious (possibly due e.g. to an unrecognized cosmic ray hit affecting the profile of Fe II $\lambda 6149.2$ ). However, it may also indicate that maximum field strength has just been passed. This will have to be confirmed by future observations, especially because it is not quite consistent with Preston's (1971a) estimate of the field strength $(5.6 \mathrm{kG})$ from differential magnetic broadening of spectral lines. In any case, the period of HD 50169 appears to be much longer than 4 years. 
This is consistent with the slow variation of the longitudinal field reported by Babcock (1958). The only recent determination of this field moment, $+1.3 \mathrm{kG}$ (Mathys \& Hubrig 1996) falls within the range of values derived by Babcock.

Between our first and last observations of HD 50169 (4 years apart), its radial velocity has monotonically increased, by about $2 \mathrm{~km} \mathrm{~s}^{-1}$ : thus HD 50169 appears to be a spectroscopic binary with an orbital period significantly longer than 4 years.

\subsection{HD 55719}

HD 55719 is one of only three magnetic Ap stars known to be SB2. This property has been discovered by Bonsack (1976), who has determined the orbital parameters and has also obtained 24 measurements of the longitudinal magnetic field. From 22 of them, ranging roughly from +1 to $+2 \mathrm{kG}$, he derived possible values of the rotation period of 30.39 and 36.48 . Regardless of the period, Bonsack's (1976) remaining two longitudinal field measurements $(-1.1$ and $-0.6 \mathrm{kG})$ cannot be reconciled with the bulk of his magnetic data.

We have already discussed the properties of HD 55719 in Papers I (where the discovery of resolved magnetically split lines was first reported) to III. We now have 29 measurements of its mean magnetic field modulus. They show no significant correlation with the phases computed using the two values of the period indicated by Bonsack (1976). Since Bonsack's (1976) data marginally indicated that the period would not be very long, we first tried to fit our mean field modulus measurements with periods in the range $0.5-100 \mathrm{~d}$, without success: the corresponding periodogram looks like pure noise. On the other hand, plotting against Julian Date our data of the two consecutive observing seasons during which we have observed the star most intensively (from October 1992 to May 1994) suggests the existence of a systematic modulation with a periodicity of the order of 2 years or more (see Fig. 14). This urged us to extend the frequency analysis to longer periods. We found, indeed, that in a periodogram covering the range $100-2000 \mathrm{~d}$, a double peak clearly stands out, indicating that the rotation period of HD 55719 may well have one of the two values:

$P_{1}=(847 \pm 20) \mathrm{d}$

or

$P_{2}=(775 \pm 25) \mathrm{d}$.

The magnetic field modulus is plotted against the phases computed with these two possible values of the period in Figs. 15 and 16. No choice between them can be made on the basis of the presently available data.

Figures 17 and 18 show phase diagrams of Bonsack's (1976) mean longitudinal field measurements obtained with the tentative periods derived from the mean field modulus data. Except for the two negative field values (unexplained by any plausible period), and to a lesser extent, for the third smallest measurement $(+760 \mathrm{G}$, which tends also to be an outlier with Bonsack's values of the periods), these measurements are not violently inconsistent with the long periods proposed here. They do, in fact, seem to show some trend with these periods (especially $847 \mathrm{~d}$ ). Three more recent measurements of the longitudinal field, performed from observations carried out at ESO with the $3.6 \mathrm{~m}$ telescope and the Zeeman analyzer of CASPEC by Mathys \& Hubrig (1996), yielded values between 450 and $950 \mathrm{G}$, that is, smaller than the bulk of Bonsack's data. However, such inconsistencies between longitudinal field data obtained at different sites and with different instruments are not unusual (see e.g. Mathys 1991). Only when more measurements are obtained with CASPEC can they be used (possibly in combination with Bonsack's data) to constrain the value of the rotation period.

That the rotation period of HD 55719 is long receives further support from the fact that the star undergoes no significant photometric variations over a timescale of the order of one month (Heck et al. 1987). However, more data need to be obtained before its value can be indisputably established.

It should finally be noted that the components of the line Fe II $\lambda 6149.2$ in HD 55719 have unusual, quite asymmetric shapes (see Fig. 2), with rather steep edges toward the line centre and more extended wings outwards (on the red side of the red component and on the blue side of the blue component). This distortion cannot be attributed to the contribution of the secondary component of the binary to the observed spectrum (see Papers I and II). It hints at a rather unusual structure of the magnetic field.

\subsection{HD 59435}

HD 59435 is also an SB2. This property has been discovered by North (1994). It is probably the most interesting of the three SB2s known to comprise a magnetic Ap star, since both components have rich, sharp-lined spectra, so that both of them can be studied in great detail. This system has been thoroughly discussed in a separate paper (Wade et al. 1996b). Here we shall just summarize some of the main results of this work.

The orbital period of HD 59435 is $1387 \mathrm{~d}$. The primary is a G giant, while the secondary is the Ap star, which must be close to the end of its main-sequence life.

The line Fe II $\lambda 6149.2$ of the Ap component is resolved into its magnetically split components. At many orbital phases, this line is blended with the same or another line of the other component. This complicates the determination of the mean field modulus: the spectrum of the Ap star first has to be rebuilt by removing the contribution of the other component. Details of the procedure are given by Wade et al. (1996b). 


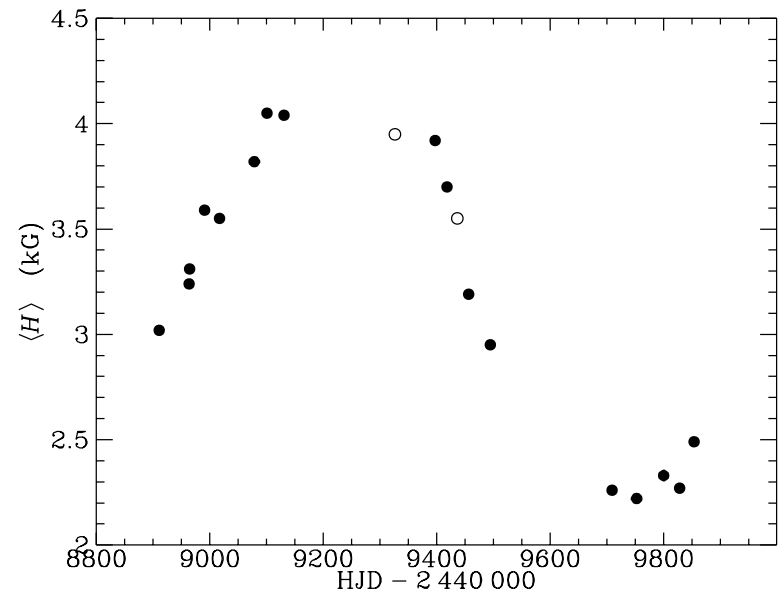

Fig. 19. Mean magnetic field modulus of HD 59435 against heliocentric Julian date. The meaning of the symbols is as given in Table 5

The mean field modulus of the Ap component is plotted against Julian date in Fig. 19. A slow variation is very clearly seen. The data obtained until now seem to indicate that the rotation period may be slightly longer than $1000 \mathrm{~d}$. But this must be taken with caution, since the magnetic field measurements performed so far do not quite cover this time span. In any case, it is already clear that the amplitude of variation of the mean field modulus of HD 59435 is remarkably large: the ratio between the maximum and minimum strengths found until now, 1.8, is among the largest found in any star with resolved lines known. Also, the field modulus at minimum (hardly more than $2200 \mathrm{G}$ ) is the smallest one measured in any of the stars considered in this paper.

No measurements of the mean longitudinal magnetic field of HD 59435 have been published. But Babcock (1967) had reported it to be magnetic on the basis of observations of circular polarization in spectral lines.

\subsection{1. $H D 61468$}

Our most recent discovery of a star with magnetically resolved lines is HD 61468. Very little is known about this faint, cool Ap star. Until now, we have obtained four measurements of its mean magnetic field modulus, spanning 119 days. These measurements are consistent with a slow monotonic decrease of the field intensity during that time interval. The radial velocity was also found to vary, with an amplitude of at least $45 \mathrm{~km} \mathrm{~s}^{-1}$, so that the star definitely is SB. The orbital period cannot be determined yet, of course, but it must be significantly shorter than 119 days.

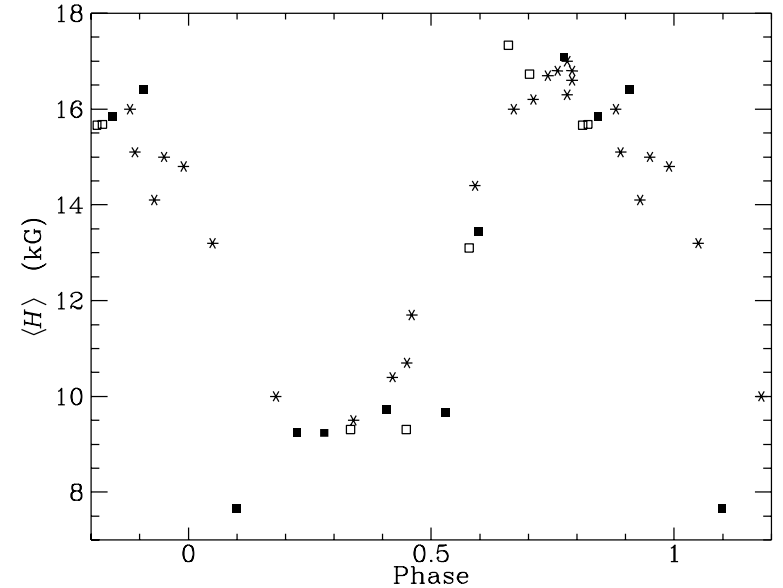

Fig. 20. Mean magnetic field modulus of HD 65339 against rotation phase. Asterisks represent Huchra's (1972) data; the meaning of the other symbols is as given in Table 5

\subsection{HD 65339}

HD $65339(=53 \mathrm{Cam})$ is possibly the Ap star whose magnetic field has been most studied. The presence of resolved lines in its spectrum has been first reported by Preston (1969b), and Huchra (1972) has been the first one to take advantage of it to try to model the magnetic field structure. The most extensive sets of measurements of the longitudinal field have been published by Babcock (1958) and by Borra \& Landstreet (1977). According to the latter authors, the longitudinal field varies between -5.4 and $+4.2 \mathrm{kG}$ along the stellar rotation period of 8.0267 . When phased together according to this period, Huchra's (1972) measurements of the mean magnetic field modulus and ours agree well (see Fig. 20). The extrema of the field modulus variations coincide, to the achieved accuracy, with those of Borra \& Landstreet's (1977) longitudinal field curve. Our data for 53 Cam show a unusually large scatter around a smooth variation curve, reflecting the relatively large errors of our measurements. Indeed, the line Fe II $\lambda 6149.2$ appears poorly suited to the diagnosis of the field modulus of that star: the combination of rotational Doppler effect and of particularly strong blending on the blue side makes the separation of its components (especially the wavelength of its blue component) very difficult to determine accurately. In particular, near phase 0.1, the components of Fe II $\lambda 6149.2$ can hardly be recognized, and we derive a very discrepant field value. Accordingly, our new data at best confirm that Borra \& Landstreet's (1977) value of the period adequately matches data spanning a timebase of nearly 40 years. But they do not significantly contribute otherwise to an improved knowledge of the magnetic field of 53 Cam. 


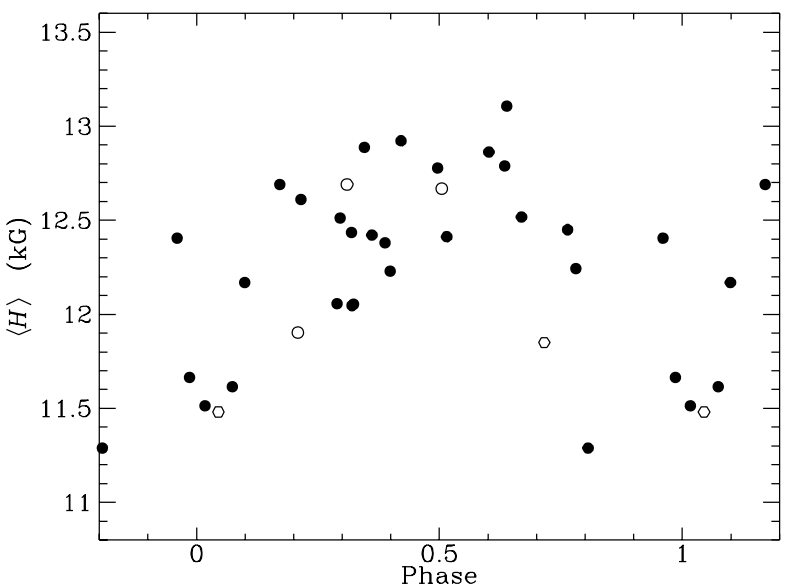

Fig. 21. Mean magnetic field modulus of HD 70331 against rotation phase, computed assuming that the rotation period is 3.0308. The phase origin is HJD 2446987.10. The meaning of the symbols is as given in Table 5

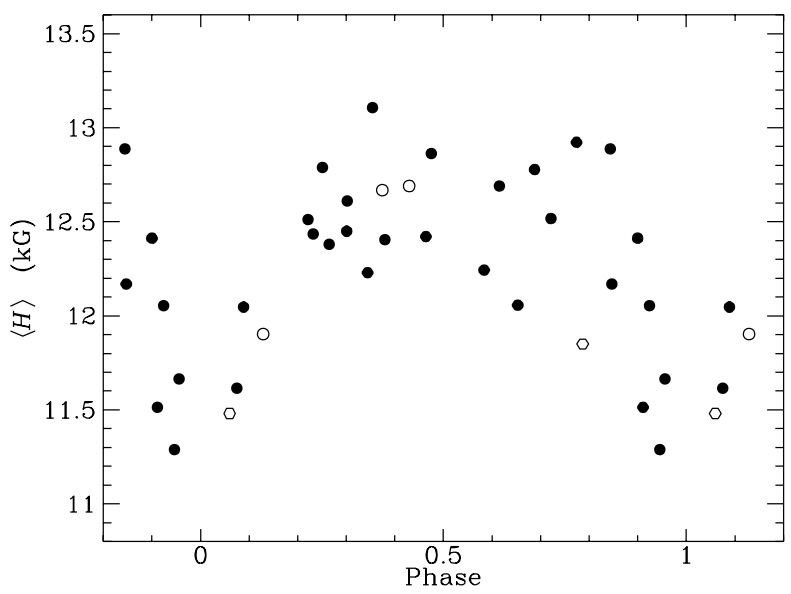

Fig. 22. Mean magnetic field modulus of HD 70331 against rotation phase, computed assuming that the rotation period is 3 . 6515 . The phase origin is HJD 2447000.60. The meaning of the symbols is as given in Table 5

Let recall that HD 65339 is a spectroscopic binary, which has been studied in detail by Scholz \& Lehmann (1988). Its orbital period is long: $2432 \mathrm{~d}$.

\subsection{3. $H D 70331$}

HD 70331, in which the observation of magnetically resolved lines is reported here for the first time, is one of the hottest stars presently known to have this property. Due to this high temperature, the line Fe II $\lambda 6149.2$ is rather weak. It is furthermore quite distorted by rotational Doppler effect, so that the diagnosis of the mean magnetic field modulus cannot be done with high accu-

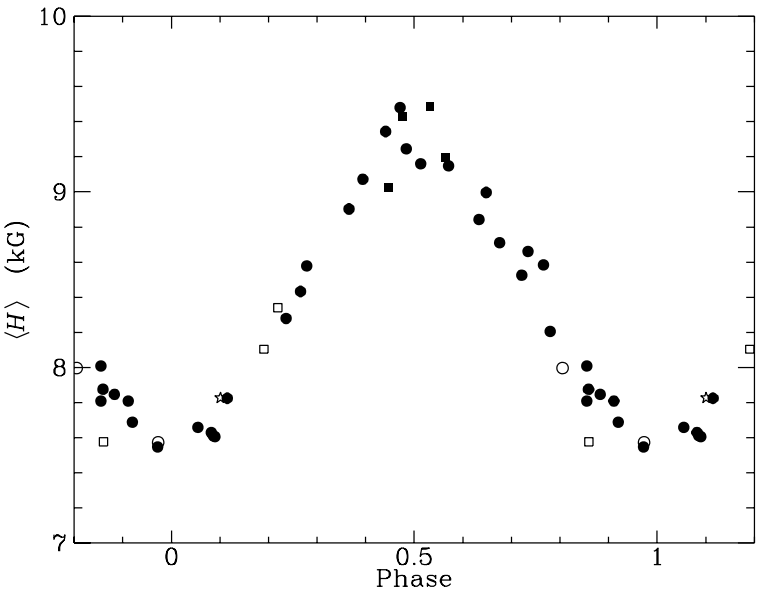

Fig. 23. Mean magnetic field modulus of HD 81009 against rotation phase. The meaning of the symbols is as given in Table 5

racy. This probably explains at least in part why we were not able to determine unambiguously the rotation period of this star. There is little doubt that the latter is short, thus that the star is seen almost pole on. Possible, but still very questionable values are $(3.0308 \pm 0.0005) \mathrm{d}$ (see Fig. 21) or $(3.6515 \pm 0.0007)$ d (see Fig. 22).

The only determination of the longitudinal magnetic field of HD 70331 performed so far yielded a value of $-2.8 \mathrm{kG}$ (Mathys \& Hubrig 1996).

\subsection{HD 75445}

We report here for the first time the presence of magnetically resolved lines in HD 75445. The standard deviation of the 9 measurements of the mean magnetic field modulus of this star is $42 \mathrm{G}$. This somewhat exceeds the estimated uncertainty of our magnetic measurements: the latter should be at most $30 \mathrm{G}$, given the good $S / N$ ratio of our spectra and the purity of the profile of Fe II $\lambda 6149.2$. It seems likely that we have recorded small but real variations of the field. But for the time being, we do not have enough data to describe them fully: it only appears that the rotation period of the star might be significantly shorter than the 450 days covered by our observations.

\subsection{HD 81009}

HD 81009 has already been extensively discussed in Papers I through III. The presence of resolved magnetically split lines in its spectrum had first been noticed by Preston (1971a). The value of its rotation period derived from our 39 magnetic measurements alone,

$P=(33.96 \pm 0.02) \mathrm{d}$,

is in perfect agreement with that obtained from photometry by Waelkens (1985). The phase coverage of our 


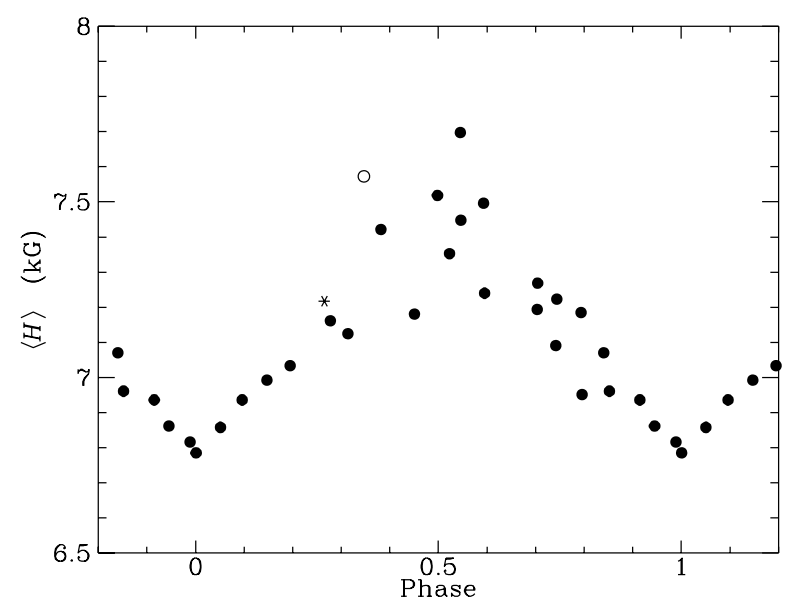

Fig. 24. Mean magnetic field modulus of HD 93507 against rotation phase. The meaning of the symbols is as given in Table 5

observations is excellent. The variation of the mean field modulus shows some anharmonicity (Fig. 23): the shape of the variation curve near maximum is almost triangular, while it is broader and flatter around minimum.

18 determinations of the longitudinal field, well distributed throughout the rotation cycle, have been recently performed by G. Hill and D. Bohlender, using the technique of $\mathrm{H} \beta$ photopolarimetry (Hill, private communication). The variation curve is definitely anharmonic, with $\left\langle H_{\mathrm{z}}\right\rangle$ raising slowly from minimum (of the order of $600 \mathrm{G}$, close to phase 0.0) to maximum (about $2000 \mathrm{G}$ around phase 0.65), and a much steeper slope back from maximum to minimum. Mathys \& Hubrig's (1996) only measurement of $\left\langle H_{\mathrm{z}}\right\rangle$ (1900 $\mathrm{G}$ at phase 0.706$)$ agrees well with the data of Hill \& Bohlender.

\subsection{HD 93507}

HD 93507 is another star that has been very little studied until now, for which the resolution of magnetically split lines is reported here for the first time. Its rotation period can be determined without ambiguity from our 28 measurements of the mean field modulus:

$P=(556 \pm 22) \mathrm{d}$.

The variation of the field modulus with this period is shown in Fig. 24. It is probably anharmonic, in particular with a triangular shape of the curve at field minimum. The larger scatter of the measurements close to field maximum is due to the fact that Fe II $\lambda 6149.2$ is considerably weaker around this phase, and that its blue component is heavily blended, making its wavelength very difficult to determine accurately (see Fig. 25).

Mathys \& Hubrig (1996) have determined the mean longitudinal field of HD 93507 finding values of 1.6 and $2.6 \mathrm{kG}$ at phases 0.609 and 0.911 , resp.

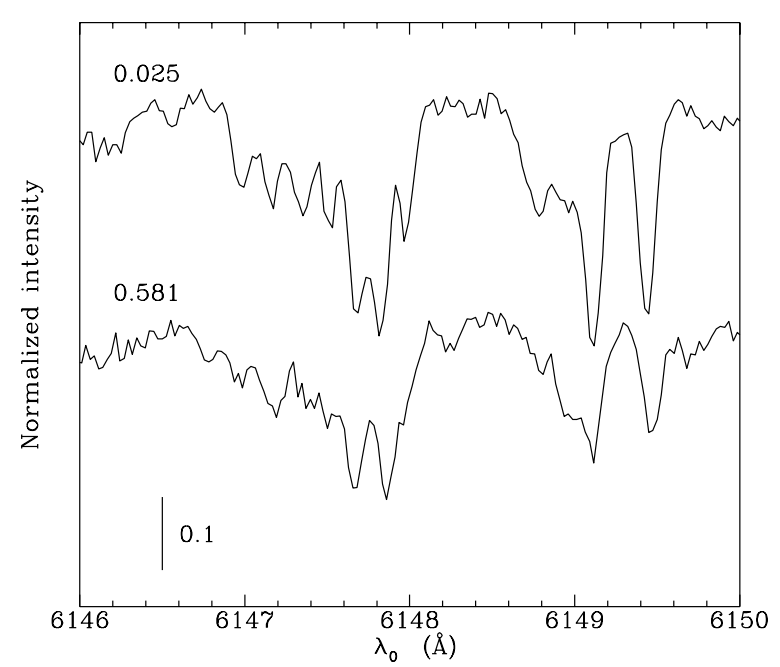

Fig. 25. Same spectral region as in Fig. 1, as observed in HD 93507 at the phases indicated next to each tracing. Note that the Fe II lines $\lambda 6147.7$ and $\lambda 6149.2$ are significantly weaker around phase 0.5 than around phase 0.0

\subsection{HD 94660}

HD 94660 has been observed for more than 7 years within the framework of this programme since the discovery of its having magnetically resolved lines (Paper I). The corresponding measurements of its mean field modulus are shown in Fig. 26. From their consideration, the rotation period of the star appears to be of the same order as the length of the time interval during which we have followed it. This is consistent with Hensberge's (1993) suggestion, based on photometric observations, that this period may be close to $2700 \mathrm{~d}$. In any case, the variation of the mean field modulus appears very anharmonic.

The four measurements of the longitudinal field of HD 94660 carried out by Mathys (1994b) and Mathys \& Hubrig (1996) from ESO CASPEC circular polarization observations span a time interval of $2500 \mathrm{~d}$, similar to that covered by the field modulus data. The longitudinal field does not appear to have varied significantly during that time, when it was always of the order of $-2.0 \mathrm{kG}$. This implies that, if the stellar rotation period is indeed of the order of 7 years, the longitudinal field of HD 94660 must be essentially constant. No variation of this field moment was found either by Bohlender et al. (1993), from $4 \mathrm{H} \beta$ polarimeter measurements spanning 1200 days, which all yield values close to $-2.5 \mathrm{kG}$. Since these measurements are contemporaneous with those of Mathys (1994b) and Mathys \& Hubrig (1996), the difference between the two sets can be safely ascribed to the different measurement techniques. Such discrepancies between $\mathrm{H} \beta$ and CASPEC measurements are not unusual indeed (Mathys 1991). Similarly, the $-3.3 \mathrm{kG}$ 


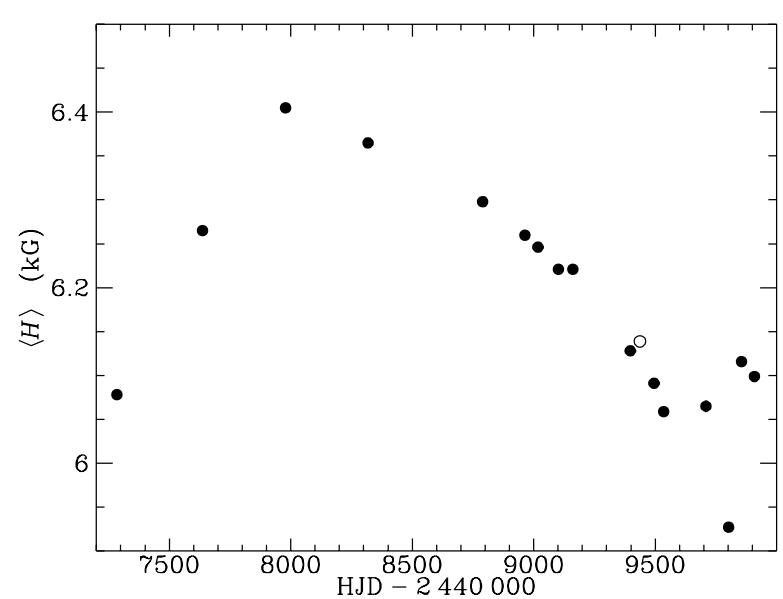

Fig. 26. Mean magnetic field modulus of HD 94660 against heliocentric Julian date. The meaning of the symbols is as given in Table 5

value derived by Borra \& Landstreet (1975) through $\mathrm{H} \alpha$ photopolarimetry is, given its estimated uncertainty of $0.5 \mathrm{kG}$, only marginally different at most from the measurements of Bohlender et al. (1993), all the more because there may be some scaling error between the $\mathrm{H} \alpha$ and $\mathrm{H} \beta$ data.

The radial velocity of HD 94660 is definitely variable. The determination of the orbital elements of this spectroscopic binary is beyond the scope of the present paper. But it can be noted that its orbital period should not be much longer than 2 years: that is, it is much shorter than the stellar rotation period.

\subsection{HD 110066}

The 4 observations obtained so far of HD 110066, in which the presence of magnetically resolved lines has first been reported in Paper III, cover only about $1 / 5$ of its probable rotation period of $4900 \mathrm{~d}$ (Adelman 1981). They are therefore mostly inconclusive as far as the field variability is concerned, all the more because two of them were performed with AURELIE and the other two with the KPNO coudé spectrograph (the existence of systematic differences between AURELIE data and measurements made with other instruments is discussed in relation with other stars). It can just be noted that the four measurements cluster around $4.1 \mathrm{kG}$, while Preston (1971a) had estimated a surface field of $3.6 \mathrm{kG}$ from differential line broadening - again, there may be systematic differences between this technique and measurements of line splitting.

Five measurements of the longitudinal field, obtained over an interval of 2 years, have been published by Babcock (1958). None of them exceeds $300 \mathrm{G}$. A null measurement was also obtained recently through $\mathrm{H} \beta$ photopolarimetry.

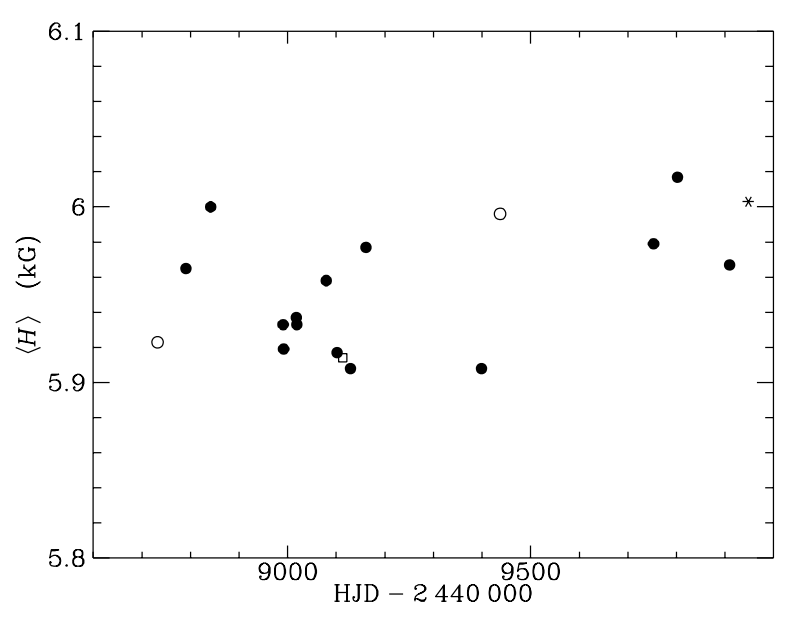

Fig. 27. Mean magnetic field modulus of HD 116114 against heliocentric Julian date. The meaning of the symbols is as given in Table 5

\subsection{HD 116114}

As for HD 110066, the discovery of resolved magnetically split lines in HD 116114 has been reported in Paper III. The 18 measurements of the mean field modulus performed since the discovery time are shown in Fig. 27. For a long time, it seemed that this field moment did not undergo any significant variation. But recent observations show hints of a slow increase, suggesting that we have just witnessed the field minimum in a star with a period much longer than 3 years. The standard deviation of the whole set of measurements, $50 \mathrm{G}$, is rather large for one of the stars where the field diagnosis is easiest and should be achievable with the best accuracy. This view is furthermore supported by the fact that if the 1995 data are excluded from the set of measurements, the standard deviation of the latter is reduced to $31 \mathrm{G}$.

The only determination of the longitudinal magnetic field of HD 116114 obtained so far yielded a value of $-1.9 \mathrm{kG}$ (Mathys \& Hubrig 1996).

The radial velocity of HD 116114 has been slowly, monotonically increasing from 4 to $7 \mathrm{~km} \mathrm{~s}^{-1}$ between our first and last observations: the star appears to be a spectroscopic binary with a period longer than 3.5 years. The low amplitude of the variations and the long timescale over which they occur probably explain why they were not detected by Abt \& Willmarth (1994).

\subsection{HD 116458}

HD 116458 is the first star with resolved magnetically split lines that has been discovered within the framework of the present programme (Papers I and II). Its rotation period, 147.9 , has been determined by Hensberge (1993) from photometric observations and shown to be consistent with 


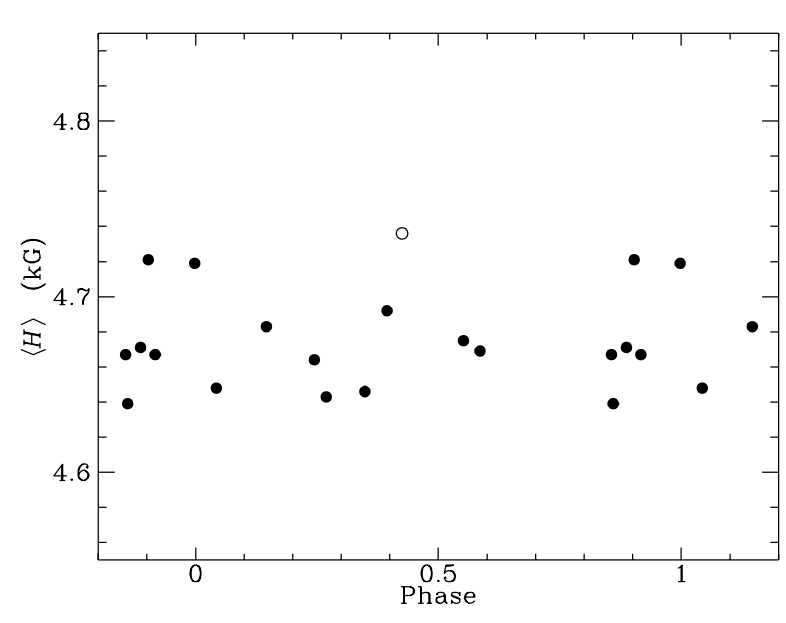

Fig. 28. Mean magnetic field modulus of HD 116458 against rotation phase. The meaning of the symbols is as given in Table 5

the longitudinal field data of Albrecht et al. (1977) and of Mathys (1991). The revision of the latter by Mathys (1994b) does not question this conclusion, which is also consistent with the more recent measurements of Mathys \& Hubrig (1996). The longitudinal field appears to vary between -1.4 and $-2.4 \mathrm{kG}$.

In contrast, the field modulus shows no significant variation. The standard deviation of our 15 measurements of this quantity is only $30 \mathrm{G}$, one of the smallest of the whole sample of stars studied here. It can be seen in Fig. 28 that these measurements are well distributed throughout the stellar rotation cycle.

It may also be noted that this star is a spectroscopic binary with an orbital period (126. 18 , Dworetsky 1982) shorter than its rotation period.

\subsection{HD 119027}

We report here for the first time the observation of resolved magnetically split lines in HD 119027. It is the faintest star having this property presently known. Furthermore, it is one of the stars of the present sample with the weakest Fe II lines. For these reasons, our field modulus determinations for HD 119027 are probably somewhat less accurate than those of other stars that we have studied. But there is little doubt that the large standard deviation ( $162 \mathrm{G}$ ) of our twelve measurements of this star reflects the observation of actual variability. Our data are insufficient to establish the period of these variations. However, significant changes seem to occur on timescales of the order of one month. On the other hand, HD 119027 is a rapidly oscillating Ap star in which Martinez et al. (1993) did not detect any significant photometric modulation due to rotation over 19 days. It seems that the only way to reconcile this with our own data is to assume that the star may have a period of rotation of the order of a few weeks, but that its surface brightness is fairly homogeneous.

\subsection{2. $H D 126515$}

HD 126515 is also known as Preston's star, since Preston's (1970) discovery that it has resolved magnetically split lines. This is one of the four stars for which, before this work, the variations of the mean field modulus had been studied throughout the rotation period. The latter, as a matter of fact, had been determined by Preston (1970) from his field modulus measurements, spanning a timebase of 12.5 years. The value that he had found, $130 \mathrm{~d} 0$, is consistent with the value of $(129.99 \pm 0.04) \mathrm{d}$ determined by North \& Adelman (1995) from Geneva and Strömgren photometric observations collected between 1971 and 1994.

When we plot Preston's (1970) and our mean magnetic field modulus measurements together against phase, there appears to be a systematic intensity shift between the two sets. In order to obtain a quantitative estimate of it, we fitted the variation with phase of each set separately by a sinusoid, using North \& Adelman's (1995) value of the rotation period. The mean value over the stellar rotation period of the field modulus determined in that manner from Preston's (1970) data is $13485 \mathrm{G}$, while from our measurements it is $12735 \mathrm{G}$ : hence the two sets differ systematically by $750 \mathrm{G}$. Such a difference, which is of the order of $6 \%$ of the mean field value, can probably be explained by systematic effects (e.g., difference of instrumental polarization) between the measurements performed on different spectral lines with different telescopes and spectrographs.

After subtracting $750 \mathrm{G}$ from Preston's (1970) data, we combined them with our data and performed a period search on the whole set. The value of the period derived in that way, from measurements spanning 38 years, is:

$P=(129.95 \pm 0.02) \mathrm{d}$,

which is slightly more accurate but fully consistent with North \& Adelman's (1995) value. Both Preston's (1970) measurements (after subtraction of $750 \mathrm{G}$ ) and our data are plotted against the phase computed with this period in Fig. 29. Figure 30 is a phase diagram, based on the same period, of all the published measurements of the mean longitudinal field (Babcock 1958; Preston 1970; van den Heuvel 1971; Mathys 1994b; Mathys \& Hubrig 1996). A very remarkable property of HD 126515 appears from the comparison of these two figures: the curve of variation of its longitudinal field is extremely asymmetric, while the variation of its mean field modulus is much less anharmonic. This behaviour, which is similar to that of HD 81009 (see Sect. 5.15), is at odds with those of other stars studied in this paper for which enough 


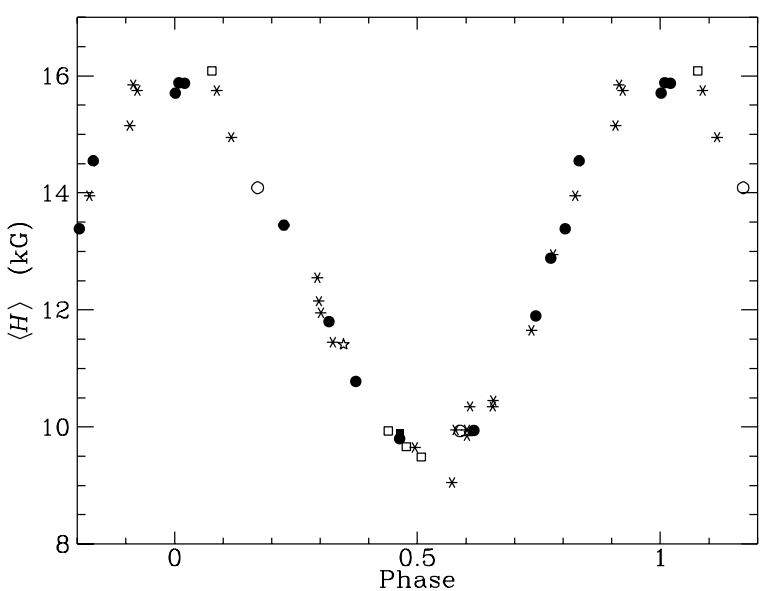

Fig. 29. Mean magnetic field modulus of HD 126515 against rotation phase. Asterisks represent Preston's (1970) measurements, shifted by $-750 \mathrm{G}$. The meaning of the other symbols is as given in Table 5

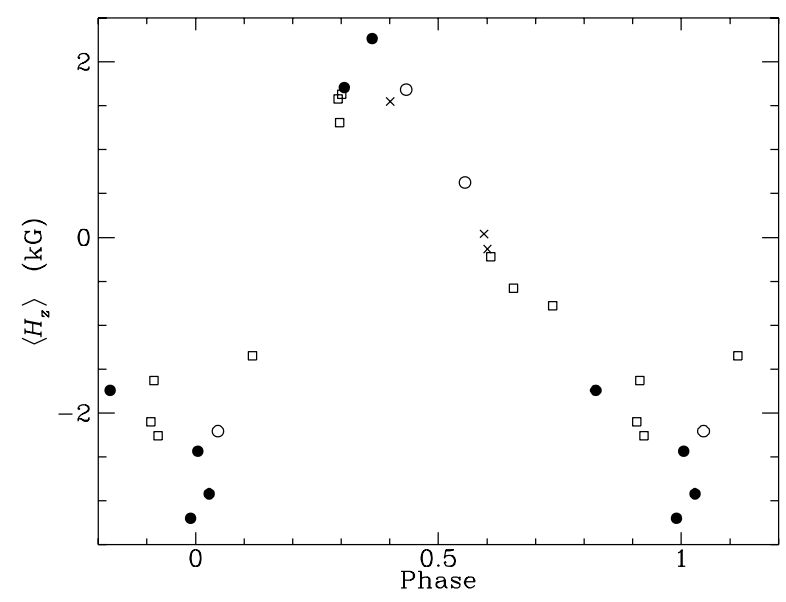

Fig. 30. Mean longitudinal magnetic field of HD 126515 against rotation phase. Data are from Preston (1970; open squares), van den Heuvel (1971; crosses), Mathys (1994b; filled circles) and Mathys \& Hubrig (1996; open circles)

longitudinal field determinations are available: in all of them, the longitudinal field varies nearly sinusoidally, while the field modulus may show significant anharmonicity.

\subsection{HD 134214}

We had reported in Paper III the observation of resolved magnetically split lines in HD 134214. Our 26 measurements of the magnetic field modulus of this star, obtained at ESO and at KPNO, are all clustered around $3.1 \mathrm{kG}$, with a standard deviation of $65 \mathrm{G}$. Since our spectra of this fairly bright star have a good $S / N$ ratio and since

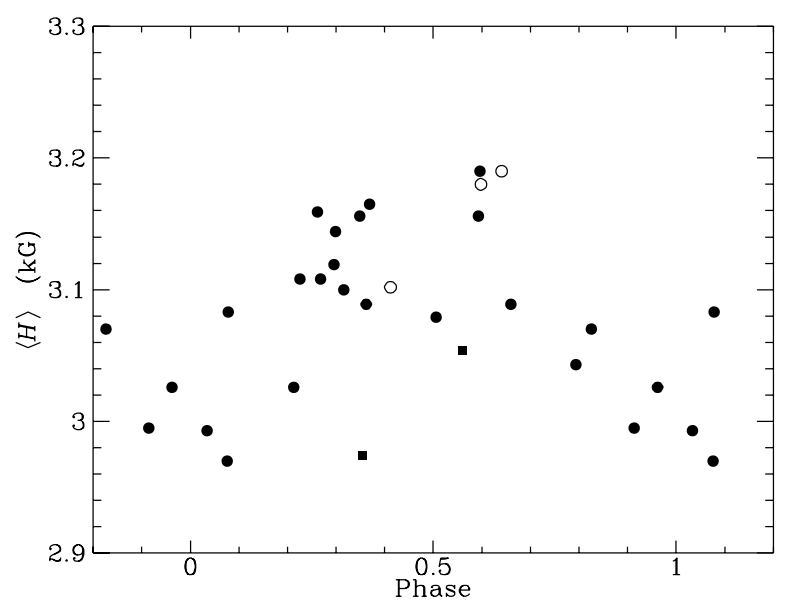

Fig. 31. Mean magnetic field modulus of HD 134214 against rotation phase, computed assuming that the rotation period is 4. 1456 . The phase origin is HJD 2447018.10. The meaning of the symbols is as given in Table 5

Fe II $\lambda 6149.2$ is not heavily blended, we estimate that the uncertainty of our field modulus determinations is significantly lower than this standard deviation, hence that we are detecting actual variations. Kreidl et al. (1994) were unable to establish the rotation period of this rapidly oscillating Ap star from their photometric observations. A period search conducted over our entire dataset yielded a most probable value of 12 d 844 . It is obviously spurious, as it would correspond to a very peculiar distribution in phase of the observations: a cluster of scattered measurements over 0.2 cycle, and a field constant throughout the rest of the period. However, given the small amplitude of the variations, the period search could easily be frustrated by small systematic instrumental effects. To probe this eventuality, we repeated it on the ESO CAT + CES data alone. The standard deviation of these 24 measurements is $63 \mathrm{G}$. The 12.844 period still appears, but a second peak corresponding to

$$
P=(4.1456 \pm 0.0014) \mathrm{d}
$$

also stands out in the periodogram. This period gives a much more convincing match to the ESO data, but in order for the two KPNO measurements to be consistent with it, one must assume that they are systematically smaller than those of ESO by about $100 \mathrm{G}$ (see Fig. 31). This cannot be definitely ruled out, but is not easily reconciled with the absence of large systematic difference between the KPNO and ESO data for the other stars observed from both sites. It can be noted that if a shift of $+100 \mathrm{G}$ is applied to the KPNO measurements, the standard deviation of the whole set is slightly reduced, to $61 \mathrm{G}$.

In any event, the value of the period suggested above must be regarded at best as tentative. Should it be the 
actual period of rotation of HD 134214, that we see magnetically resolved lines would imply that the star's rotation axis makes a very small angle with the line of sight, a possibility also contemplated by Kreidl et al. (1994). On the other hand, the period analysis performed on the ESO data alone does not allow one to exclude even shorter rotation periods. Nevertheless, the constraint that they would impose on the geometry of the observation would be even more stringent. In relation with this, it may also be stressed that no significant Doppler effect is definitely seen in the line profiles.

Two attempts by Mathys \& Hubrig (1996) to measure the longitudinal magnetic field of HD 134214 gave null results.

\subsection{HD 137909}

HD $137909(=\beta \mathrm{CrB})$ is one of the brightest and best studied Ap stars. The presence of magnetically resolved lines in its spectrum has first been reported by Preston (1969c). Systematic determinations of the mean magnetic field modulus throughout the rotation period have been carried out by Wolff \& Wolff (1970). They have subsequently been used by various authors, in combination with longitudinal field measurements, to derive constraints on the magnetic geometry of this star (see e.g. Landstreet 1980 and references therein).

We have obtained a new set of measurements of the mean magnetic field modulus of $\beta \mathrm{CrB}$ from observations performed at OHP with the AURELIE spectrograph and at KPNO. In Fig. 32, they are plotted together with Wolff \& Wolff's (1970) data against the rotation phase calculated according to Kurtz's (1989) ephemeris. Our measurements are seen to be in good agreement with those of Wolff \& Wolff (1970) close to magnetic maximum, but the amplitude of the variations seemed larger from the latter than it appears from our data. This discrepancy is probably mostly spurious, as indicated by the large scatter of Wolff \& Wolff's (1970) data around field minimum. This scatter likely results from the difficulty of measuring the rather weak field of $\beta \mathrm{CrB}$ around that phase on photographic spectra restricted to the blue region (where the Zeeman splitting is smaller than in the red).

Considering our observations alone in Fig. 32, the mean field modulus variations are seen to be strongly anharmonic, with a rather sharp raise toward a fairly narrow maximum and a smoother decrease toward a broader minimum. However, an inconsistency between the KPNO and OHP measurements, significantly larger than the internal scatter of each of these separate sets about a smooth variation curve, is apparent. Because the KPNO measurements were obtained contemporaneously with the OHP data interleaved with them, the discrepancy cannot be attributed to an inaccuracy of the value of the period used nor to the occurrence of secular intrinsic changes in the stellar field. Also the good internal consistency of each of

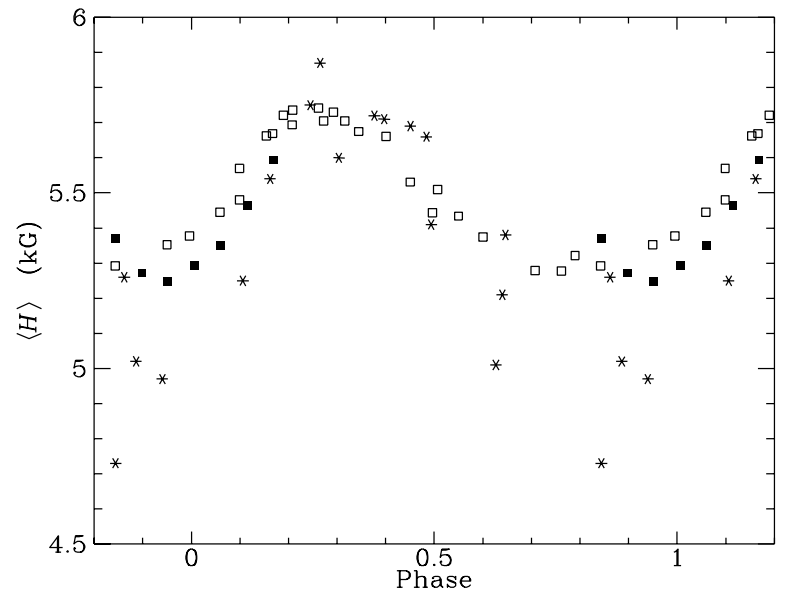

Fig. 32. Mean magnetic field modulus of HD 137909 against rotation phase. Asterisks represent Wolff \& Wolff's (1970) measurements. The meaning of the other symbols is as given in Table 5

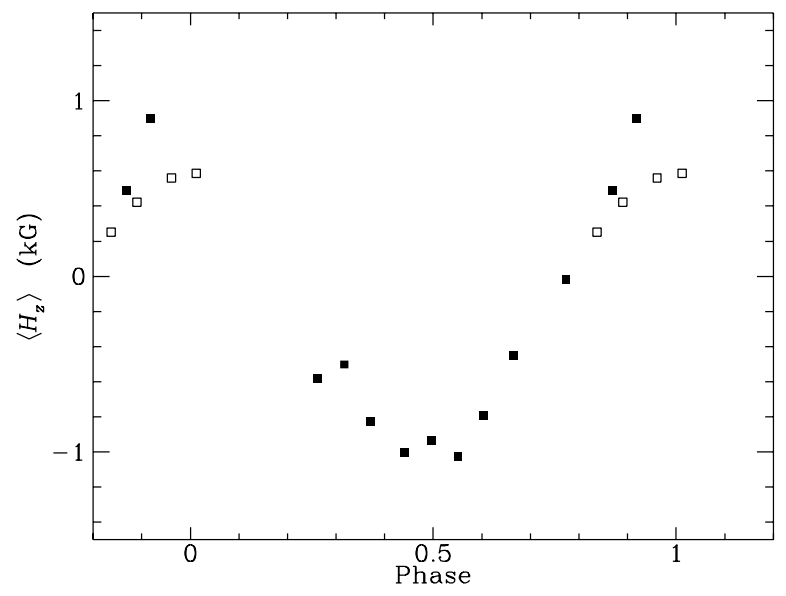

Fig. 33. Mean longitudinal magnetic field of HD 137909 against rotation phase. Data are from Mathys (1994b; filled squares) and Mathys \& Hubrig (1996; open squares)

the datasets taken independently (for instance, as we mention in Sect. 6, the rms deviation of the AURELIE data about a fit by a sine and its first harmonic is $28 \mathrm{G}$ ) rules out the possibility of short timescale intrinsic variations of the stellar magnetic field. The most likely interpretation of the observed different behaviour of the measurements conducted at OHP and at KPNO is that it results from a systematic instrumental effect. As this effect appears to depend on the rotation phase of the star, it is most probably related to instrumental polarization. This is of course worrisome, since if this explanation is correct, the shape of the curve of variation of the mean field modulus may depend on the instrument used to obtain it, which limits its usefulness for the diagnosis of the geometrical 
Table 6. The mean longitudinal magnetic field of HD 137949

\begin{tabular}{lllrr}
\hline Reference & $\begin{array}{l}\text { JD of first } \\
\text { and last } \\
\text { observation } \\
-2400000\end{array}$ & $n$ & $\begin{array}{c}\text { Average } \\
\left\langle H_{\mathrm{z}}\right\rangle(\mathrm{G})\end{array}$ & $\begin{array}{c}\text { Standard } \\
\text { deviation } \\
(\mathrm{G})\end{array}$ \\
\hline Babcock (1958) & 36003 & 1 & +1120 & 47 \\
van den Heuvel (1971) & $40315-40412$ & 5 & +1380 & 285 \\
Wolff (1975) & $41458-41844$ & 9 & +1569 & 149 \\
Mathys \& Hubrig (1996) & $48782-49026$ & 2 & +1834 & 95 \\
\hline
\end{tabular}

structure of the stellar magnetic field. This point is discussed in more detail in Sect. 6 .

Notwithstanding this, there is no doubt that, as already pointed out by Wolff \& Wolff (1970), there is a very significant phase lag between the extrema of the mean field modulus and those of the longitudinal field: compare Fig. 32 with Fig. 33, where the longitudinal field measurements of Mathys (1994b) and of Mathys \& Hubrig (1996) have been plotted against rotation phase. This implies that the field of $\beta \mathrm{CrB}$ is not symmetric about an axis passing through the centre of rotation of the star: this star shows an extreme example of this property which appears widespread among Ap stars (e.g., Mathys 1993). Modeling of the geometry of its field using the data presented here as well as Leroy's (1995b) broadband linear polarization measurements is under way, following the approach pioneered by Leroy (1995a). Preliminary results have been presented by Wade (1995).

\subsection{5. $H D 137949$}

The mean magnetic field modulus of HD 137949 has not significantly varied since our discovery that this star has magnetically resolved lines in March 1991 (Paper II). Our 13 determinations of it over almost 4.5 years, all performed from spectra taken at ESO with the CAT and the CES, have a standard deviation of only $23 \mathrm{G}$. This gives a good idea of the accuracy and reproducibility that are achieved in the measurements reported in this paper.

The absence of detectable variation of the mean field modulus is consistent with the lack of photometric variability in more than 7 years of observation (Deul \& van Genderen 1983), with the lack of detectable rotational sidelobes in the pulsation frequency spectrum of this rapidly oscillating Ap star (Kurtz 1991), and with the constancy of its broadband linear polarization over 3 years (Leroy 1995b).

The 23.26 rotation period inferred by Wolff (1975) from the consideration of her and van den Heuvel's (1971) longitudinal field measurements is probably not significant. Indeed, the 5 measurements of van den Heuvel (1971), spanning 97 days, have a standard deviation of $285 \mathrm{G}$, of the same order as their probable error: in other words, no significant variation is detected. Similarly, al- though Wolff (1975) does not quote the uncertainty of the nine longitudinal field determinations that she obtained in one year, their standard deviation of $149 \mathrm{G}$ is of the same order as the typical uncertainty of such measurements based on photographic spectra. The average and the standard deviation of all the longitudinal field determinations performed by various authors for HD 137949 are summarized in Table 6 . The columns are, in order, the reference of the paper where the data are published, the first and last date of observation, the number of measurements found in the considered reference, their average, and their standard deviation. For Babcock's (1958) single measurement, the latter is replaced by the estimated uncertainty. Under the hypothesis that indeed no short-term variation is detected in van den Heuvel's (1971) and Wolff's (1975) data, all the existing measurements of the longitudinal field of HD 137949 are consistent with a slow monotonic increase of this quantity, at a rate of $\sim 20 \mathrm{G} / \mathrm{yr}$, since its first determination by Babcock (1958).

This, combined with the other pieces of evidence mentioned above, strongly supports the view that HD 137949 is rotating very slowly. Under the assumption that its longitudinal field varies roughly sinusoidally (which is not unusual among Ap stars), its rotation period might be of more than 75 years: thus it would be of the same order as or longer than the period of $\gamma$ Equ, until now the longest one of any known Ap star (see Sect. 5.38). Further measurements, in particular of the longitudinal field, of HD 137949, will be most useful to confirm the suggestion made here.

\subsection{HD 142070}

Magnetically resolved lines have been discovered in HD 142070 in 1994 . That this star is rotating rather quickly was soon recognized from the contribution of Doppler effect to the profile of Fe II $\lambda 6149.2$ (clearly seen in Fig. 3) and the occurrence of significant variations of its mean field modulus from night to night. To this date, 22 measurements of the latter have been accumulated. Their frequency analysis unambiguously shows that the rotation period of HD 142070 is

$P=(3.3748 \pm 0.0012) \mathrm{d}$. 


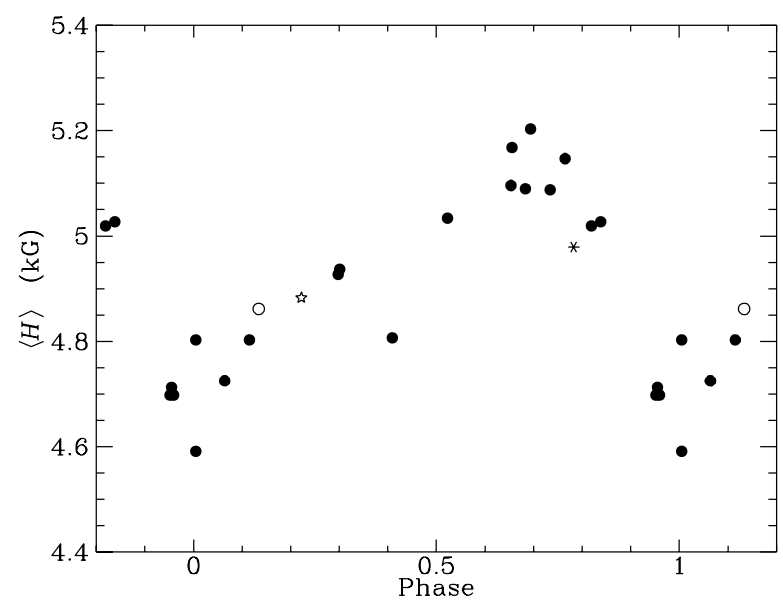

Fig. 34. Mean magnetic field modulus of HD 142070 against rotation phase. The meaning of the symbols is as given in Table 5

This makes HD 142070 the star with resolved magnetically split lines with the shortest unambiguously determined rotation period. This implies that the star must be observed almost pole-on. Indeed, from the width of the resolved line components, $v \sin i$ is constrained to be smaller than $5 \mathrm{~km} \mathrm{~s}^{-1}$, approximately. Assuming that the stellar radius is at least $2.4 R_{\odot}$, we derive for the inclination of the rotation axis on the line of sight an upper limit $i \lesssim 8^{\circ}$. That in these conditions the modulus of the magnetic field varies by almost $10 \%$ of its average value over a rotation cycle indicates that this field must be very inhomogeneous. Note also the very asymmetric shape of the variation curve of the the mean field modulus (see Fig. 34).

Between our first and last observations of HD 142070, its radial velocity has smoothly varied with a total observed amplitude of $2.5 \mathrm{~km} \mathrm{~s}^{-1}$. The amplitude of the variation that has taken place since our first observation must be larger, though, as the radial velocity must have passed through a minimum between July 1994 and February 1995, when we have no spectra. In any case, our observations of this spectroscopic binary only cover part of its orbital period. Accordingly, this period must be significantly longer than 500 days.

\subsection{7. $H D 144897$}

Very little is known about HD 144897, a star in which the presence of magnetically resolved lines is reported here for the first time. The measurement of the field modulus in this star is difficult and not very accurate, due to heavy blending of Fe II $\lambda 6149.2$ on both its blue and red wings. Nevertheless, thanks to the fairly large amplitude of variation of the field modulus, the rotation period can

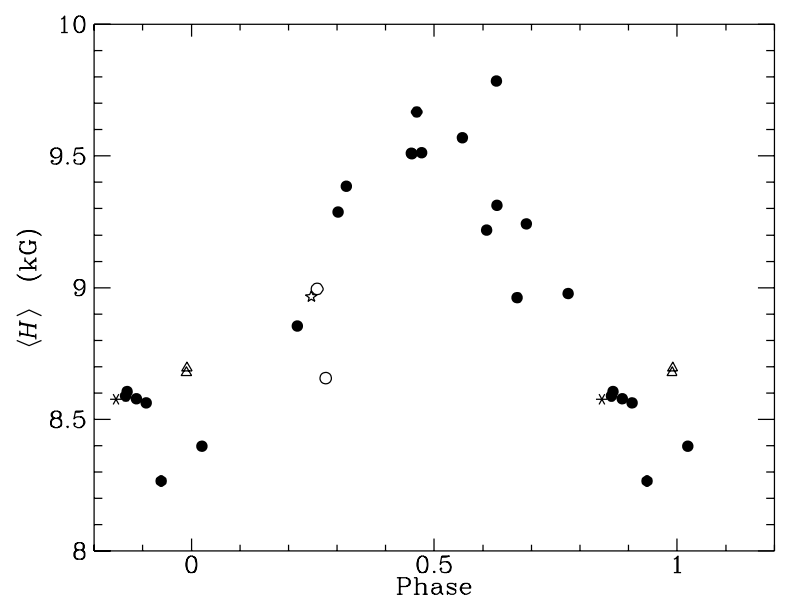

Fig. 35. Mean magnetic field modulus of HD 144897 against rotation phase. The meaning of the symbols is as given in Table 5

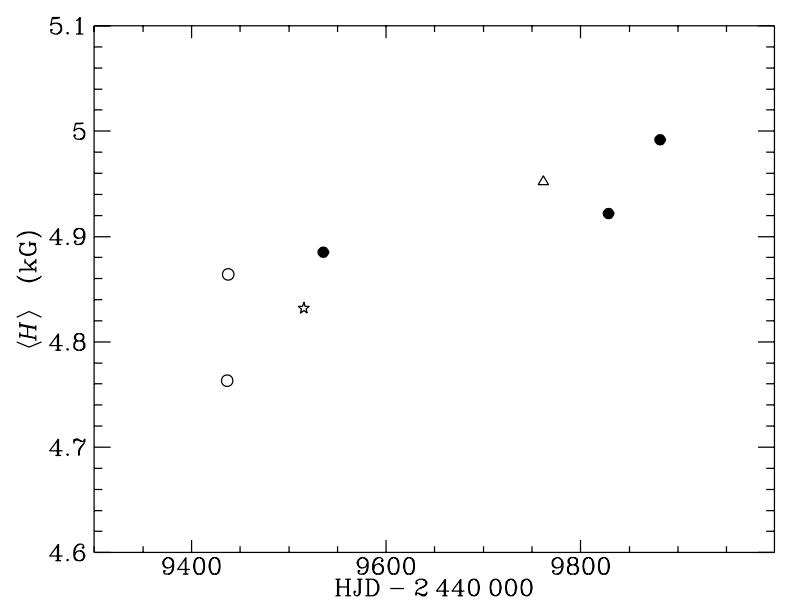

Fig. 36. Mean magnetic field modulus of HD 150562 against heliocentric Julian date. The meaning of the symbols is as given in Table 5

be unambiguously determined from our 26 measurements of this quantity:

$P=(48.43 \pm 0.14) \mathrm{d}$.

The corresponding phase diagram is shown in Fig. 35. Due to the above-mentioned difficulty of measuring the field modulus accurately, the shape of its curve of variation is not well defined. It however appears less anharmonic than that of other stars.

One measurement of the longitudinal magnetic field of HD 144897 by Mathys \& Hubrig (1996) yielded a value close to $+2.0 \mathrm{kG}$ at phase 0.794 . 


\subsection{HD 150562}

About all that was known of HD 150562 until now is that it is rapidly oscillating (Martinez \& Kurtz 1994). We report here that it has spectral lines resolved into their magnetically split components. We have observed this star for a little more than one year. During this time, its mean field modulus has shown a slow, monotonic increase (Fig. 36). Thus the rotation period must be significantly longer than 1 yr.

\subsection{HDE 318107}

The peculiarity of HDE 318107 has been discovered by North \& Cramer (1981) through a study of the open cluster NGC 6405 in Geneva photometry. The observations reported here seem to be the first spectroscopic observations of this star, from which we report the detection of magnetically resolved lines. Due to its faintness, most of our spectra of this star are rather noisy (the $S / N$ ratio is most often of the order of 70). Furthermore, Fe II $\lambda 6149.2$ is rather severely blended, especially on the blue side. Accordingly, our measurements of the mean magnetic field modulus are not very accurate: we estimate that their uncertainty is of the order of $300 \mathrm{G}$. This is still significantly less than the standard deviation of the whole set of measurements, $1065 \mathrm{G}$ : the latter must reflect real variations. They appear inconsistent with the value of the rotation period favoured by North (1987) from a study of photometric variability. Reanalyzing the photometric data (kindly made available by P. North) together with our magnetic measurements, we were unable to find a single period consistent with both types of observations. Obviously, more data are required. Consideration of the longitudinal field may prove very helpful in that respect. One measurement of that moment has been reported by Mathys \& Hubrig (1996): $+2.0 \mathrm{kG}$.

\subsection{HD 165474}

Resolved magnetically split lines have been discovered in HD 165474 by Preston (1971a), who determined that the field modulus was $7.2 \mathrm{kG}$ from the observation of Zeeman doublets. This value seems significantly larger than our own measurements, which range from 6.1 to $6.8 \mathrm{kG}$. However, from magnetic broadening of unresolved lines, Preston (1971a) had derived a field strength of $6.6 \mathrm{kG}$, more consistent with our data. The latter have a standard deviation of $139 \mathrm{G}$. This almost certainly indicates that we are detecting real field variations, since HD 165474 appears as one of the most favourable cases for the accurate measurement of the mean field modulus. Our measurements are inconsistent with the two values of the rotation period favoured by Leroy (1995b) from his broadband linear polarization measurements $(23.38$ and 12.02). Accordingly, we performed a frequency analysis

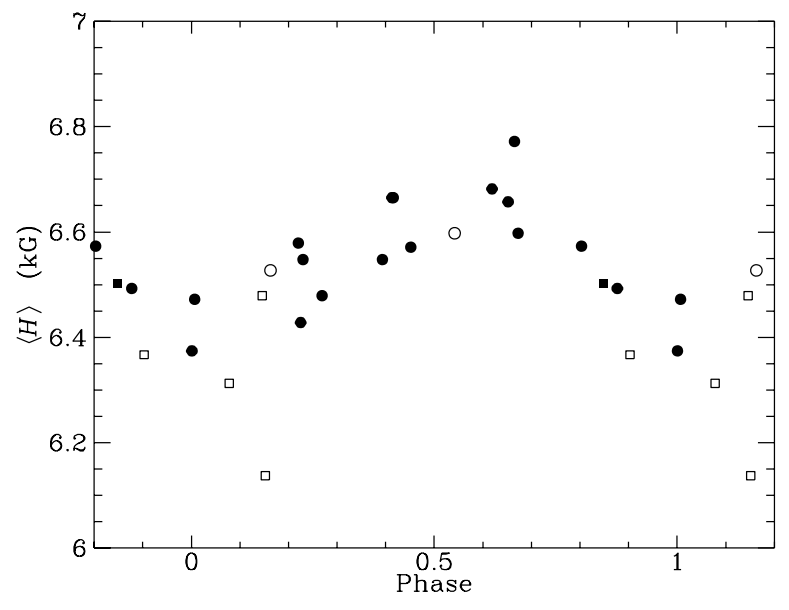

Fig. 37. Mean magnetic field modulus of HD 165474 against rotation phase, computed assuming that the rotation period is 2.54065. The phase origin is HJD 2447000.0. The meaning of the symbols is as given in Table 5

on our data alone. One value of the rotation period seems to stand out rather clearly:

$P=(2.54065 \pm 0.00020) \mathrm{d}$.

The corresponding phase diagram is shown in Fig. 37. One measurement is seen to show a large deviation: it corresponds to a very noisy spectrum, which may explain its behaviour. On the other hand, the ESO, OHP and KPNO data all appear reasonably consistent with this period. But because of the small amplitude of the variations, and because the visibility of magnetically resolved lines requires a rather peculiar geometry of observation with such a short period, we feel that this value of the period must be confirmed by additional observations before it can be regarded as definitive. Leroy's (1995b) linear polarization data are not useful in that respect, due to their unsuitable distribution in time. Published longitudinal field measurements, on the other hand, are somewhat puzzling: while Babcock (1958) had reported a non-negligible positive value $(+900 \mathrm{G})$, three null measurements have been obtained by Mathys (1994b) and by Mathys \& Hubrig (1996), the first one in 1988 and the last two in 1992, at an interval of two days. This appears to be purely coincidental: preliminary visual examination of a spectrum recorded recently (end of July 1996) in both circular polarizations clearly indicates the unquestionable presence of a quite sizeable longitudinal field. Thus $\left\langle H_{\mathrm{z}}\right\rangle$ in HD 165474 turns out to show significant variability. Additional determinations of this field moment should consequently prove most useful to derive a definite value of the rotation period. 


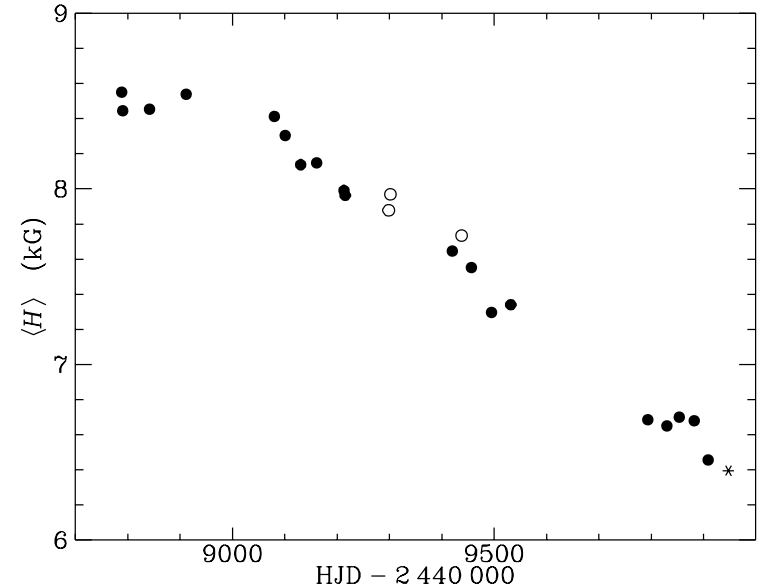

Fig. 38. Mean magnetic field modulus of HD 166473 against heliocentric Julian date. The meaning of the symbols is as given in Table 5

\subsection{HD 166473}

We report here the discovery of the presence of resolved magnetically split lines in the rapidly oscillating (Kurtz \& Martinez 1987) Ap star HD 166473. Our measurements of the magnetic field modulus of this star are plotted against Julian date in Fig. 38. It can be seen that the field was at its maximum at the time of discovery of the resolved lines. It remained fairly constant during our first observing season, and since then, it has been steadily decreasing. The relative amplitude of the variation is definitely one of the largest among the stars considered here. Minimum does not seem to have been reached yet: the rotation period must significantly exceed the time spanned by the observations obtained until now (3.2 yr).

Three measurements of the longitudinal field over a time interval of 64 days are reported by Mathys \& Hubrig (1996): all yielded a field close to $-2.1 \mathrm{kG}$.

\subsection{2. $H D 177765$}

HD 177765 is another star about which almost nothing is known. Since we found that it has magnetically resolved lines, we have obtained six measurements of its mean field modulus, spanning almost two years. Their standard deviation is only $19 \mathrm{G}$ : the field has shown no variation during the interval covered by the observations. HD 177765 may well be another star with a very long period: this should be established by future observations.

\subsection{3. $H D 187474$}

Didelon $(1987,1988)$ has discovered the presence of resolved magnetically split lines in HD 187474. This star has a period of rotation of $2345 \mathrm{~d}$, derived by Mathys (1991) from his own and Babcock's (to be also found in

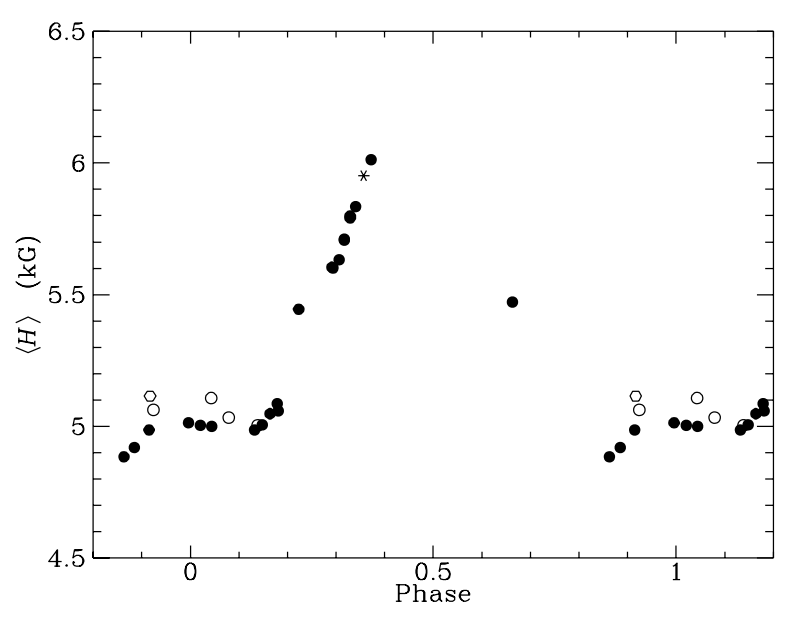

Fig. 39. Mean magnetic field modulus of HD 187474 against rotation phase. The meaning of the symbols is as given in Table 5

Mathys 1991) longitudinal field measurements. This field moment varies between -1.8 (at phase 0.5 ) and $+1.8 \mathrm{kG}$ (at phase 0.0 ). The value of the period derived from its consideration is supported by photometric observations (Hensberge 1993). Our magnetic field modulus data are plotted against the phase computed using Mathys' (1991) ephemeris in Fig. 39. The anharmonicity of the variation curve is remarkable, all the more because the variation of the longitudinal field is almost perfectly sinusoidal (see Fig. 33 of Mathys 1991). Observations in the next two years will be important, as they will fill the gap between phases 0.37 and 0.66 in the field modulus curve, and as they should in particular constrain the maximum of this quantity.

Note also that HD 187474 is a spectroscopic binary with an orbital period of $690 \mathrm{~d}$ (Leeman 1964), much shorter than its rotation period.

\subsection{HD 188041}

Preston (1971a) had been the first to observe magnetically resolved lines in HD 188041. Our 15 measurements of its field modulus have a standard deviation of $37 \mathrm{G}$, part of which appears to be due to the detection of real variations of very low amplitude (probably not much more than $50 \mathrm{G}$ peak-to-peak). Indeed, there is some hint in Fig. 40 at a systematic variation of the mean field modulus with the phase computed with the rotation period of 223.9 derived by Hensberge (1993). The field modulus appears to be maximum close to phase 0 , and minimum close to phase 0.5 . These extrema are close to those of the longitudinal field (see Fig. 36 of Mathys 1991). Measurements of the latter have been performed by Babcock $(1954,1958)$, Wolff (1969), Mathys (1994b), and Mathys \& Hubrig (1996). It is always positive and varies with a peak-to-peak 


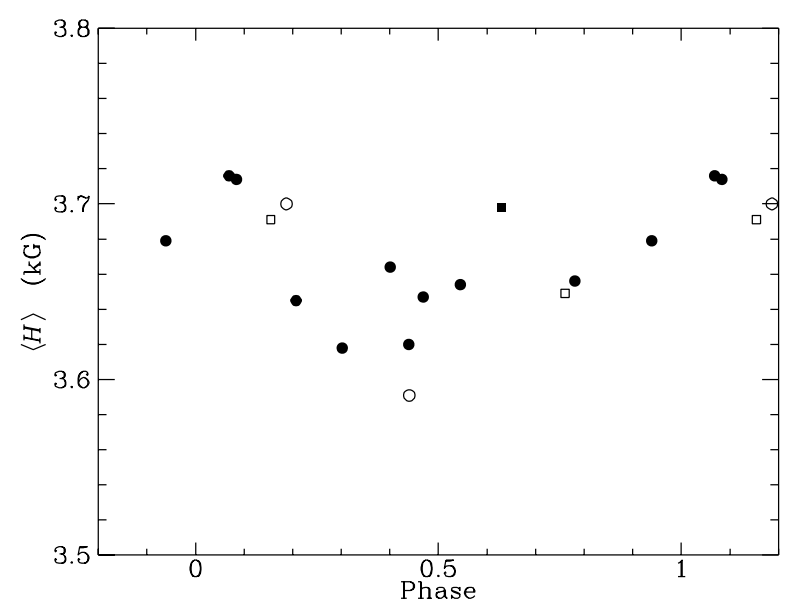

Fig. 40. Mean magnetic field modulus of HD 188041 against rotation phase. The meaning of the symbols is as given in Table 5

amplitude not much larger than $0.5 \mathrm{kG}$, which is qualitatively consistent with the low amplitude of the field modulus variations. Note also that significantly variable broadband linear polarization is measured in this star (Leroy 1995b).

\subsection{5. $H D 192678$}

From his broadband linear polarization measurements, Leroy (1995b) has derived a value of 6.4186 for the rotation period of HD 192678, a star in which the detection of magnetically resolved lines has first been reported in Paper II. Our magnetic field modulus measurements show a definite trend with this period, but there appears to be a systematic shift between those obtained at OHP with AURELIE and those obtained at KPNO. Since both sets are well distributed throughout the rotation cycle, this difference can be estimated just from the comparison of the mean of the measurements of each set. For the 20 OHP measurements, this mean is $4831 \mathrm{G}$, while for the 14 KPNO points, it is $4668 \mathrm{G}$. Accordingly, in order to bring all the data to the same scale, we subtracted $163 \mathrm{G}$ from each OHP measurement.

We then performed a period search on the whole set of our measurements, modified as just explained. Two values of the period stand out: 5.6237 , which is quite inconsistent with Leroy's (1995b) linear polarization data and must thus be ruled out, and $(6.4185 \pm 0.0040) \mathrm{d}$, which is in excellent agreement with Leroy's (1995b) value. The latter is used to compute the phases of our observations. The resulting phase diagram is shown in Fig. 41.

As for other short-period stars discussed in this paper, the axis of rotation of HD 192678 must make a small angle to the line of sight, so that resolved lines can be observed despite the rather fast rotation. As a matter of

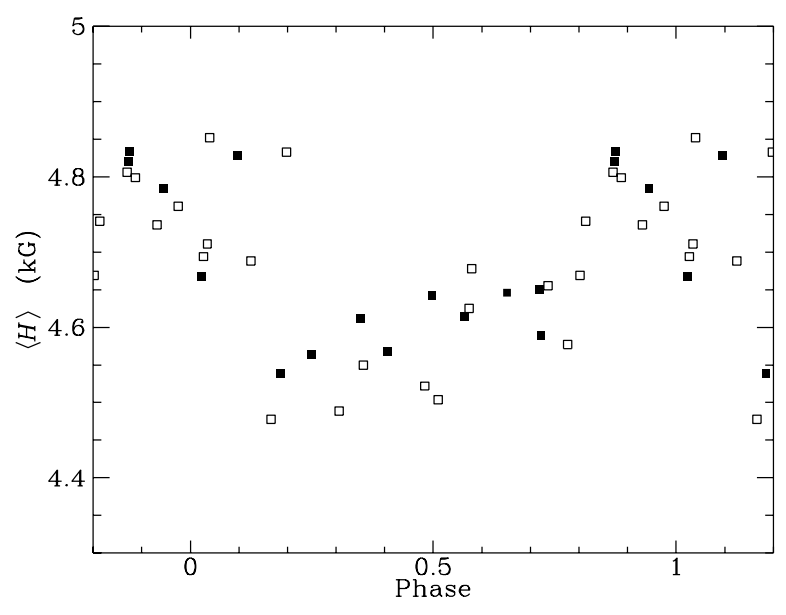

Fig. 41. Mean magnetic field modulus of HD 192678 against rotation phase. The meaning of the symbols is as given in Table 5. The AURELIE data have been shifted by $-163 \mathrm{G}$

fact, Doppler distortion is definitely seen in the split components of Fe II $\lambda 6149.2$. A detailed analysis of the magnetic geometry of this star, taking into account not only the present field modulus data and Leroy's linear polarization measurements, but also the longitudinal field determinations of Babcock (1958) and Glagolevskij et al. (1986), is presented in a separate paper (Wade et al. 1996a).

\subsection{HDE 335238}

The presence of resolved magnetically split lines in HDE 335238 has first been reported by Preston (1971a). The distribution of our 16 measurements of the mean field modulus of that star along its rotation period appears particularly unfortunate, since while 14 of them lie between 7.8 and $8.8 \mathrm{kG}$, the other two determinations yield much higher values: 11.2 and $11.7 \mathrm{kG}$. Although this unusual distribution of the measurements may lead one to wonder whether the two "discrepant" high field values are not spurious (which might seem even more plausible because most of our spectra of this fairly faint star are rather noisy), their correctness is unquestionable. Indeed, in the two spectra from which a field of more than $11 \mathrm{kG}$ is derived, all spectral lines do consistently show larger magnetic splitting than in our 14 other observations. As a matter of fact, Preston (1971a) had already reported field modulus values ranging from 9.1 to $11.8 \mathrm{kG}$ in $\mathrm{HDE}$ 335238 , in excellent agreement with the measurements presented here.

As a result of the poor phase distribution of our data, our attempts to determine the star's rotation period from their consideration suffer from severe aliasing problems. It appears that the period of HDE 335238 must undoubtedly be between 40 and 50 days, but there are several 


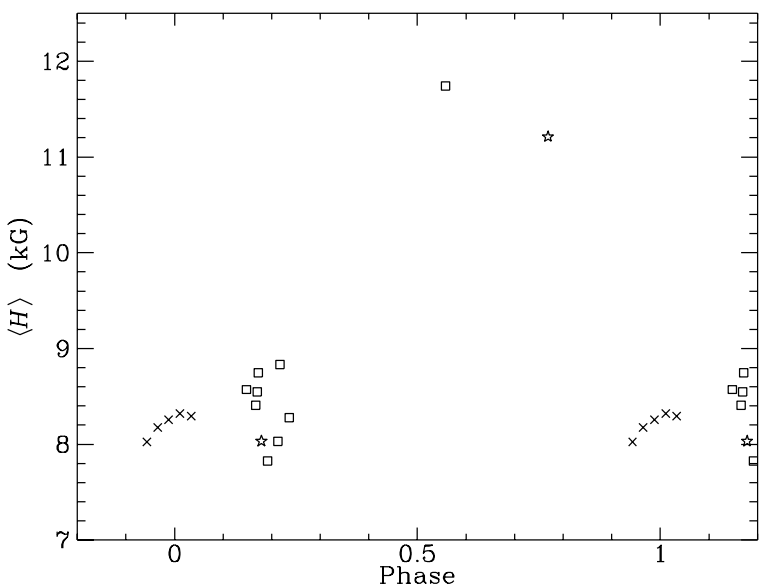

Fig. 42. Mean magnetic field modulus of HDE 335238 against rotation phase, computed assuming that the rotation period is 44. 0 . The phase origin is HJD 2447000.0. The meaning of the symbols is as given in Table 5

possibilities in that range between which one cannot unambiguously choose. The value

$P=(44.0 \pm 0.3) \mathrm{d}$

seems marginally more probable than the others (the phase diagram obtained with this period is shown in Fig. 42). But one cannot definitely rule out the aliases 49 d $4,47.9$, and 42.8 . Obviously, more observations of this star are required to settle the issue of its period.

Babcock (1967) had mentioned that HDE 335238 is magnetic, probably on the basis of spectra taken in circular polarization, but the only published determination of its mean longitudinal field has been performed by Mathys \& Hubrig (1996), who found a value of $-1.3 \mathrm{kG}$.

\subsection{HD 200311}

The first report in the literature of the presence of resolved magnetically split lines in HD 200311 is Adelman's (1974) mention of a private communication from Preston. However, this property of HD 200311 had apparently been forgotten until it came serendipitously to our attention (Paper II). The star brightness is at the limit for observation with a $0.9 \mathrm{~m}$ telescope, so that our KPNO spectra of it are rather noisy. Furthermore, since it is hot, the line Fe II $\lambda 6149.2$ is fairly weak. Therefore our mean field modulus measurements, especially those performed from KPNO spectra, are not quite so accurate as for most stars studied here. However, the amplitude of the field variations is large enough so that the stellar rotation period can be uniquely determined from the field modulus data. We find:

$P=(51.75 \pm 0.13) \mathrm{d}$.

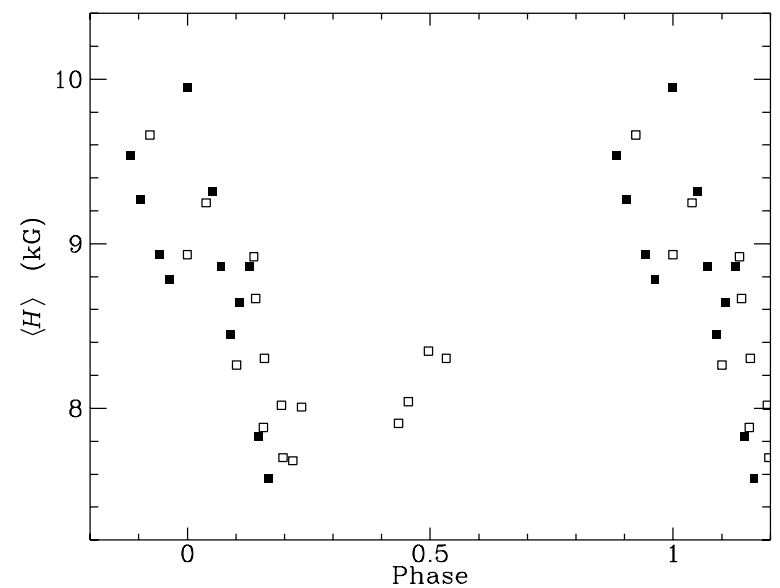

Fig. 43. Mean magnetic field modulus of HD 200311 against rotation phase. The meaning of the symbols is as given in Table 5

We used this period to build the phase diagram shown in Fig. 43. One clearly sees in this figure that the KPNO data are more scattered than those from OHP about a smooth variation curve. This reflects the limits of accuracy mentioned above.

Like HDE 335238, HD 200311 was one of the stars reported to be magnetic by Babcock (1967). More recently, measurements of its longitudinal field have been performed at the Special Astrophysical Observatory of the USSR Academy of Sciences (Glagolevskij et al. 1986) and at the Elginfield Observatory of the University of Western Ontario (to be published separately). They range, roughly, between -2.5 and $+2.5 \mathrm{kG}$ : HD 200311 appears to have a strong, polarity reversing longitudinal field.

\subsection{8. $H D 201601$}

We have confirmed in Paper I Scholz's (1979) probable detection of resolved magnetically split lines in HD 201601 $(=\gamma \mathrm{Equ})$. It is well known, mostly from the longitudinal field measurements, that this star has a period that must exceed 70 years (see e.g. Mathys 1991). It has sometimes been questioned whether this period really corresponds to the stellar rotation, or if some other effect might be involved. A compelling argument in support of the interpretation in terms of stellar rotation has recently been presented by Leroy et al. (1994), on the basis of their observations of broadband linear polarization.

The behaviour of the mean magnetic field modulus over the 7 years during which we have repeatedly measured it is also consistent with the long period mentioned above: indeed, we mainly observe a slow, monotonic increase (Fig. 44). There is some hint of a flattening of the curve in the last measurements, which may suggest that we are getting close to the field maximum. This is difficult 


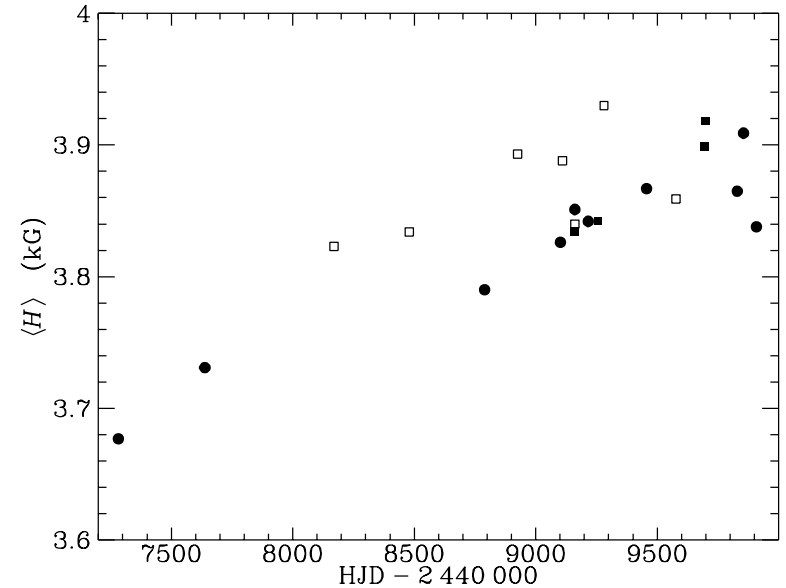

Fig. 44. Mean magnetic field modulus of HD 201601 against heliocentric Julian date. The meaning of the symbols is as given in Table 5

to ascertain though, all the more because like in other stars discussed in this paper (e.g., HD 137909, HD 192678), there also seem to be some systematic differences between measurements obtained with different spectrographs (with in particular the AURELIE data somewhat larger than the others).

\subsection{9. $H D 208217$}

In the region of Fe II $\lambda 6149.2$, the spectrum of HD 208217, in which the presence of magnetically resolved lines is reported here for the first time, bears some resemblance with that of 53 Cam (HD 65339): the lines are affected by a fairly large rotational Doppler effect, but the magnetic field is strong enough so that magnetic line splitting is observed. However, as in 53 Cam, rotation seriously complicates the determination of the mean magnetic field modulus. The difficulty is even aggravated by the fact that the blue component of Fe II $\lambda 6149.2$ is heavily blended. These circumstances severely limit the accuracy achievable in the diagnosis of the field modulus.

A first determination of the rotation period of HD 208217 has been made by Manfroid \& Renson (1983) from Strömgren photometry. New photometric observations, combined with the magnetic data reported here, have allowed Manfroid \& Mathys (1996) to refine this determination. They found

$P=(8.44475 \pm 0.00011) \mathrm{d}$,

a value which we use here to compute the phases of our observations. The resulting phase diagram for the mean magnetic field modulus is shown in Fig. 45. Though as a result of the measurement intricacies mentioned above, it is rather noisy, there is a clear indication of a double wave in the curve of variation of the field modulus

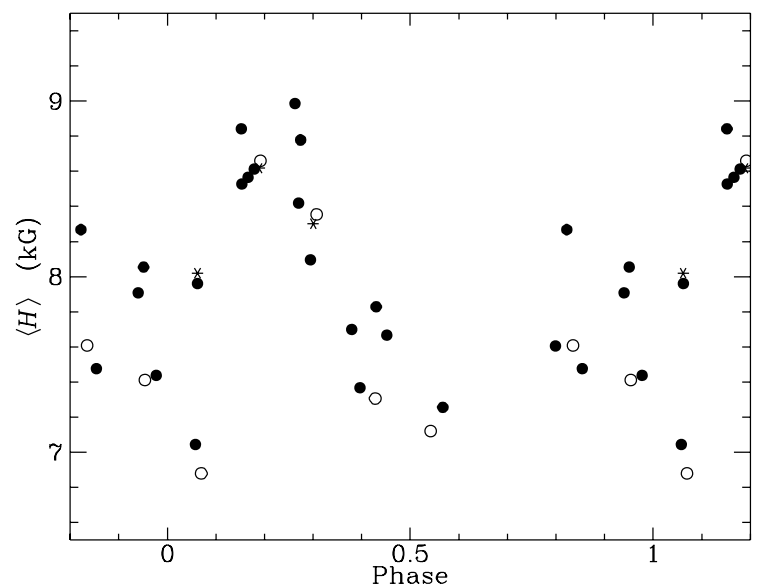

Fig. 45. Mean magnetic field modulus of HD 208217 against rotation phase. The meaning of the symbols is as given in Table 5

(Manfroid \& Mathys 1996 even found the contribution of the first harmonic to be marginally more significant than that of the fundamental). HD 208217 is the first star with resolved magnetically split lines known to show this behaviour, which should be widespread if the magnetic fields of Ap stars generally were centred dipoles. (This does not imply that the field of HD 208217 is a centred dipole.)

Our observations furthermore show that the radial velocity of HD 208217 is variable, with a peak-to-peak amplitude which is at least of the order of $20 \mathrm{~km} \mathrm{~s}^{-1}$. The orbital period of this spectroscopic binary is presently unknown. A value of 75 d 1 cannot be definitely ruled out, but it appears more likely that the period is at least of the same order as the time span of our observations (2 years).

\subsection{HD 216018}

For a long time after the discovery that HD 216018 has resolved magnetically split lines (reported for the first time here), no variation of the mean field modulus of this star has been observed. Only during the last observing season has it started to show some trend to increase. This is illustrated in Fig. 46. It appears probable that HD 216018 has a very long rotation period (much longer than the 3 years covered by our observations) and that we have first observed it close to its magnetic minimum. More observations should allow us to confirm this.

However, the long period hypothesis already receives some support from the three determinations of the longitudinal field performed by Mathys \& Hubrig (1996), 2 in 1992 on 2 consecutive nights and 1 almost one year later, which all yield essentially the same result $(+1.3 \mathrm{kG})$.

The radial velocity of HD 216018 shows a behaviour very similar to the mean field modulus: it has remained essentially constant over most of our observations, and it 


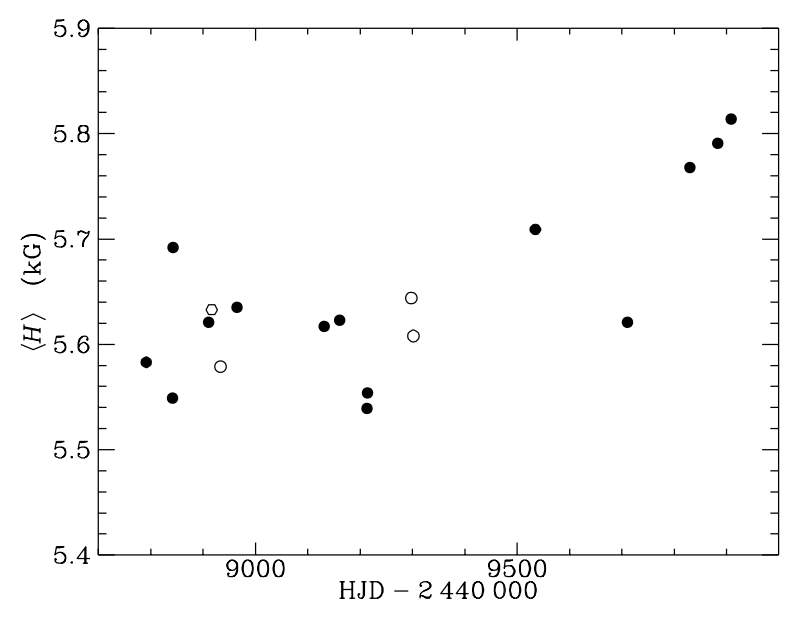

Fig. 46. Mean magnetic field modulus of HD 216018 against heliocentric Julian date. The meaning of the symbols is as given in Table 5

has started a slow decrease (by a couple of $\mathrm{km} \mathrm{s}^{-1}$ ) during the last observing season.

\section{Measurement errors}

As mentioned in Sect. 2, one of the difficulties encountered in this study is to estimate the uncertainty of the mean field modulus measurements. In many previous studies of Ap stars, the magnetic field moment of interest (mean field modulus, longitudinal field, crossover, quadratic field) was derived through a least-squares fit of measurements of some quantity characterizing the profile of a sample of spectral lines by a function of some atomic parameters. The standard error of the magnetic quantity determined through this least-squares analysis could then be used as an estimate of the internal error affecting it (see e.g. Mathys 1994b, 1995a,b). This approach cannot be applied to the present case, where the mean field modulus is diagnosed from a single line. We cannot either use the kind of argument based on photon-counting statistics that allows one to estimate the error of longitudinal field measurements based on Balmer-line photopolarimetry (Landstreet 1982). In fact, we have no way to estimate independently the uncertainty of the field modulus determinations for each studied star.

However, we can take advantage of our knowledge that the field moments for Ap stars undergo smooth, periodical variations as a result of stellar rotation. For a significant fraction of the stars studied in this paper, these variations, or at least a clear trend of the evolution of the field with time, are well defined. If we fit them by an appropriate mathematical function, we can use the rms deviation of the measurements about this fit as an estimate of the internal measurement errors for the star under consideration. Actually, we may rather expect to derive in that way an upper limit of the errors, since the mathematical function that we fit can at best be an approximate representation of the actual variation of the field modulus. Once we have estimated the measurement errors in that way for a sufficient number of stars, we can get a feeling of how they are related to the appearance of the line profiles. We can in turn use this knowledge to estimate the uncertainty of the field modulus measurements in other stars.

The results of this approach are summarized in the last two columns of Table 3 . In Col. 5 we give, for the 20 stars for which we judge that we can define sufficiently well the behaviour of the field modulus, the rms deviation of our measurements of this quantity with respect to the fit by the mathematical function that we find most appropriate to represent its variation.

For 7 of the stars (HD 2453, 12288, 81009, 93507, $144897,188041,192678)$, we used a simple sine function as an approximation to the variation curve. It must be stressed that this does not imply that we consider that the magnetic field modulus of these stars undergoes perfectly harmonic variations. Simply, the accuracy of our data does not allow us to distinguish whether there is any significant departure from harmonicity (even less so since we have no independent error estimate to compare with the scatter of the observations about a sine wave). This remark also applies to the other functions adopted to fit the variations of other stars. In the case of HD 192678, the AURELIE data have been shifted by $-163 \mathrm{G}$ before computing the fit (see Sect. 5.35), so that the scatter about the fit can be regarded as reflecting random measurement uncertainties, but not systematic instrumental errors.

For 3 stars, HD 65339, HD 137909 and HD 142070, our data are well represented by the superposition of a sine wave and of its first harmonic. The poor quality, discrepant measurement of HD 65399 that we have obtained at phase 0.098 (see Sect. 5.12) was not included in the fit, since it does not appear representative of the typical measurement uncertainty. In the case of HD 137909, we have pointed out in Sect. 5.24 the probable existence of a systematic difference between the OHP and the KPNO measurements: fitting the OHP measurements alone (by a sine wave and its first harmonic), the rms deviation was decreased to $28 \mathrm{G}$ (compared to $43 \mathrm{G}$ for the whole dataset).

For HD 126515, the superposition of a sine wave and of its first three harmonics gives the best fit (however, while the third harmonic appears significant, the second one at most marginally contributes).

On the other hand, there are several stars for which our observations cover only a fraction of the (long) rotation period, during which the field modulus variation is essentially linear. The rms deviation given in Col. 5 of Table 3 for those stars (HD 965, HD 150562, HD 201601) is computed with respect to a straight line fit. In the case of HD 201601, there is some evidence of systematic 
differences between measurements obtained at various sites (in particular, the AURELIE data seem systematically larger than those obtained with the CES and the KPNO coudé spectrograph). But this systematic difference is difficult to define reliably, especially because (as noted in Sect. 5.38) HD 201601 may be nearing its magnetic maximum. In any case, even more than for any other star, the rms deviation given in Table 3 for this star must be regarded as an upper limit of the measurement uncertainty. We also fitted a straight line to the last 19 measurements of HD 166473 (the first four show a flattening likely corresponding to the field maximum) and to the measurements of HD 50169 except for the first and the last one. The latter seems affected by an abnormally large error of unidentified origin (possibly an unrecognized cosmic ray hit on the CCD affecting the recorded line profile), while the former, which had been obtained two years before the following ones, lies above the extrapolation of the straight line fit to them. (This may well indicate that at that time, the star was around its magnetic minimum.)

As argued in Sect. 5.20, the field modulus of HD 116458 has been regarded as not undergoing any significant variation and the standard deviation of our measurements $(30 \mathrm{G})$ is taken as a good estimate of their uncertainties. We dealt in the same manner with HD 137949 and HD 177765, since we judge that we have not observed (yet?) any variation of their field modulus.

Finally, the distribution in phase of our observations of HD 200311 is not sufficiently uniform to allow us to ascertain the shape of the variation curve of its mean field modulus. However, since this star appears representative of a category of observations (somewhat noisy spectra of stars with a fairly weak line Fe II $\lambda 6149.2$, possibly affected by some blend or distortion) for which we have no better possibility to estimate the measurement uncertainties, we found important to try to get at least a rough indication of what the errors on the magnetic field modulus determinations may be for it. Therefore, we fitted our data for this star with a sine wave alone, and with superpositions of a sine wave and of up to its first three harmonics. None of these fits represents the observations significantly better or worse than the others, but the standard deviations of the data with respect to all of them fall between 320 and $355 \mathrm{G}$. Since due to its relative faintness, our KPNO spectra of this star are rather noisy (which shows up in the scatter of the measurements), we repeated the same procedure with our AURELIE data alone: the rms deviation was then down to around $260 \mathrm{G}$. For inclusion in Col. 5 of Table 3 , we adopt an intermediate approximate value of $300 \mathrm{G}$ for this star.

As mentioned in the beginning of this section, we regard the standard deviation of the measurements of the field modulus of a given star with respect to a fit of its variation by a suitable mathematical function as representative of the uncertainty affecting these measurements. Admittedly, in some cases (e.g., HD 93507: see Sect. 3.16), the measurement error shows some dependence on the rotation phase. However, this should only occur for a very small number of the stars under consideration, and even for these stars, the adoption of an average value of the uncertainty appears as the most reasonable (or even only acceptable) approach.

The smallest values of the standard deviations appearing in Col. 5 of Table 3 are of the order of $20-30$ G. They are obtained for stars in which the components of the line Fe II $\lambda 6149.2$ are well resolved, sharp and mostly symmetric (not showing any distortion by rotational Doppler effect), and not too heavily affected by blends (especially, the unknown blending line(s) frequently affecting the blue side of Fe II $\lambda 6149.2$ should not be too strong with respect to the latter). In such cases the accuracy of the measurements appears to be limited primarily by the accuracy of the wavelength calibration. Indeed, the latter can be estimated from the rms deviation of the wavelengths of the lines of the calibration arc spectrum with respect to the fitted dispersion curve. This deviation is typically of the order of $1 / 20$ to $1 / 30$ of a pixel. Taking as an example a spectrum recorded with the long camera of the ESO CES, this corresponds in wavelength units to between 0.85 and $1.3 \mathrm{~m} \AA$, or in terms of magnetic field (see Eq. (1)), to between 18 and $27 \mathrm{G}$.

As the next step, we evaluated the uncertainty of the determination of the magnetic field modulus in the stars for which the variation of this quantity is not sufficiently well defined to fit it by a mathematical function. For this, we compared the profile of Fe II $\lambda 6149.2$ in those stars with its profile in the stars discussed earlier in this section. The resulting error estimates appear in Col. 6 of Table 3 . We are confident that these estimates should be correct to within $\pm 30 \%$.

HD 59435 is the only star for which there is no entry either in Col. 5 or in Col. 6 of Table 3. On the one hand, estimating the measurement error from the aspect of the profile of Fe II $\lambda 6149.2$ may not be safe, since in many cases, this profile could only be recovered after removing the contribution of the other component of this SB2 system: possible additional errors may be introduced by this operation. On the other hand, as mentioned in Sect. 5.10, while the field modulus of this star undergoes large variations, our measurements probably do not cover a full rotation cycle yet, so that their rms deviation with respect to a mathematical fit may not be quite representative of the measurement uncertainties. However, it appears e.g. from the consideration of Fig. 19, that the latter should not exceed much $50 \mathrm{G}$.

The discussion so far has been focussed onto the random measurement errors. Our field modulus determinations may plausibly also be affected by systematic errors. The latter might introduce e.g. a constant offset of the field values, or an incorrect scaling of the measurements. Such effects affect all the determinations of the mean magnetic field modulus of a given star in essentially the same 
way: they do not show through in the dispersion of the measurements.

As a matter of fact, two sources of systematic errors can be readily identified in our measurements.

First, as mentioned in Sect. 2, the line Fe II $\lambda 6149.2$ is formed in a regime of partial Paschen-Back effect, and the interpretation of its splitting in terms of the mean magnetic field modulus through Eq. (1), which corresponds to pure Zeeman effect, is only a good approximation. The magnitude of the error that is introduced in that way depends on:

- the magnetic field strength (since departures from the Zeeman regime increase with it);

- the orientation of the magnetic field with respect to the line of sight (as this defines the relative intensities of the $\sigma$ and $\pi$ line components, which behave differently from each other in the partial Paschen-Back regime);

- the equivalent width of the line Fe II $\lambda 6149.2$ (since the degree of saturation also affects the relative intensities of the $\sigma$ and $\pi$ components).

In fact, the field strength, its orientation, and the emergent line intensity, vary from point to point on the stellar surface. But the error introduced by neglecting the departures from the Zeeman regime is, in practice, always small, and it is enough to estimate it for a simple representative case. For instance, for a magnetic field of $20 \mathrm{kG}$, the actual splitting of the $\sigma$ (resp. $\pi$ ) components is 1.021 (resp. 0.979) times the pure Zeeman splitting. Accordingly, in the stars discussed in this paper, the systematic error introduced by neglecting the departures from the Zeeman effect should never be much larger than $2 \%^{2}$.

The second type of systematic errors that is definitely present is of instrumental origin. Indeed, it has been seen in Sect. 5 that for several stars (in particular, HD 137909, HD 192678, and HD 201601), the field modulus measurements obtained from OHP AURELIE spectra are systematically different from those obtained with other instruments, in particular with the ESO CES and the KPNO coudé spectrograph. As a matter of fact, all the instrumental configurations used but AURELIE yield field determinations that are quite consistent, at the achieved level of accuracy.

Because the effect varies from star to star, as well as, for HD 137909, with rotation phase (see Sect. 5.24), we are led to think that it results from the modification of the linear polarization of the stellar light by the optical train. The resulting effect may be rather complex, since the polarization of the light emitted by the star varies across the line profile in a manner which depends on the structure of the magnetic field and on the geometry of

2 Note that the Landé factor of the upper level of Fe II $\lambda 6149.2$ is known to an accuracy better than 0.009: that is, the uncertainty that it introduces in the field measurements does not exceed $0.3 \%$. the observation. The interpretation of the observed systematic measurement differences in terms of instrumental polarization receives further support from the fact that HD 137909, HD 192678, and HD 201601 are among the stars for which Leroy (1995b) reports the definite detection of broad-band linear polarization. A more definite characterization of the effect would require the knowledge of the geometric structure of the magnetic field of the considered stars and is therefore beyond the scope of this paper.

The vast majority of the observations discussed in this paper, including those performed with AURELIE, were obtained through coudé systems. We do not believe, however, that oblique reflections on the plane coudé mirrors can account for the modification of the polarization discussed above. Otherwise, the effect would also depend on the position of the star in the sky: thus, it would show through (at least, in part) as additional scatter about the field variation curve of a given star. Such additional scatter does not appear to contribute significantly to the rms deviation of the HD 137909 AURELIE measurements about a smooth variation curve, which as reported earlier in this section, is fully consistent with the accuracy achievable in the wavelength calibration. In addition, there is no reason to suspect that the 4-mirror coudé train of the OHP $152 \mathrm{~cm}$ telescope would affect the light polarization more badly than the other coudé (or Nasmyth) systems used in this study. Some of them actually might be expected to be much more harmful to the stellar light polarization: for instance, the CFHT with its train of 7 mirrors with optimized coatings, or the alt-alt mounted ESO CAT with its 3 mirror system whose flat tertiary is frequently used at very large incidences. However, there is no indication in our data of major polarizing effect from any of these coudé systems.

It seems much more plausible that the main source of instrumental polarization rather is the spectrograph grating. With respect to this, it is noteworthy that AURELIE was equipped for our observations with a "normal" grating used in the second order, while all the other spectrographs that we employed had echelle gratings used in higher orders (that is, at high angles).

As already stressed in Sect. 5.24, the kind of systematic effect discussed here may set a rather severe limitation to our ability to determine the geometrical structure of the stellar magnetic fields, since it may modify the shape of the curve of variation of the mean field modulus (and in particular, there is some hint that it may shift the apparent phases of field extrema). Fortunately, the good consistency of the data that we obtained with all instrumental configurations but AURELIE seems to indicate that only the latter may be significantly affected by instrumental polarization.

Our data do not show any evidence of systematic errors other than those discussed above. 


\section{Discussion}

\subsection{Magnetic fields}

When the study reported here started, there were in the literature about 80 published measurements of the mean magnetic field modulus of Ap stars. Although 12 stars with magnetically resolved lines were known, almost all of those 80 measurements pertained to only four of them: HD 65339, HD 126515, HD 137909, and HD 215441. As a result, these four stars were for a long time the Ap stars for which the best knowledge of the magnetic field geometry had been achieved. However, due to their small number, it was impossible to decide confidently whether their properties were representative of those of Ap stars in general, or if they rather were isolated unusual specimens. It was even less feasible to use the limited data available to derive information of statistical nature, such as on the distribution of the field strength among Ap stars, or on the relation between field strength and other stellar properties.

The more than 750 measurements of 40 stars reported here therefore open a wide range of new perspectives. A number of general results that can be inferred from these new data at the present stage (that is, in particular, prior to any modeling effort) are discussed hereafter.

On the spectra plotted in Figs. 2 to 4, one can see that in virtually all studied stars in which the magnetic splitting of Fe II $\lambda 6149.2$ is large enough, the profile of this line comes back to (or very close to) the continuum between the two split components. There is no significant absorption at the nominal wavelength (centre) of the line, as should be the case if part of it was formed in regions devoid of a magnetic field. Since there is no evidence for extreme inhomogeneities in the distribution of iron over the surface of any of the considered stars (nor in any Ap star known), the observed spectra sample essentially the whole stellar hemisphere visible at the time of observation. Therefore, it appears that the magnetic field of the considered stars covers all (or most of) their surface or, in other words, has a filling factor (nearly) equal to 1.

Moreover, it can also be seen in Figs. 2 to 4 that, in most cases, the split components of Fe II $\lambda 6149.2$ are very sharp. This indicates that the magnetic field prevailing in the line forming region has a rather uniform strength. Indeed, if very different magnetic field intensities were found at different locations on the star, locally the lines originating from these regions of different magnetic fields would have different splittings. The observed line, which is an average of them, would as a result have broader components. We have actually mentioned in the course of the discussion of the individual stars that a few of them (HD 9996, HD 18078, HD 55719) appear from the profiles of the split components of Fe II $\lambda 6149.2$ to have magnetic fields more inhomogeneous than the bulk of the sample.

In a number of the studied stars (pointed out in Sect. 5), the profiles of the split components of the line
Fe II $\lambda 6149.2$ appear distorted by rotational Doppler effect. This distortion varies along the stellar rotation period. Probably its most characteristic signature is that, at some phases, the red component of Fe II $\lambda 6149.2$ is deeper than its blue component, which is opposite to the behaviour due to partial Paschen-Back effect alone. Rotational Doppler effect masks the sharpness of the line components. When sufficiently large compared to the line splitting, it may also result in partial overlap of the components of Fe II $\lambda 6149.2$, thus preventing them from reaching the continuum at line centre.

Besides the cases when the magnetic field is too weak to yield full splitting of sharp components and those when rotational Doppler effect broadens the components so much that they are no longer fully separated, there are two stars in which the split Fe II $\lambda 6149.2$ profile fails to come back up to the continuum at line centre: HD 166473 and HD 216018. These two cool Ap stars have very rich spectra. It may be noted in Figs. 2 to 4 that they are among the stars in which the unrecognized blue blend of Fe II $\lambda 6149.2$ is strongest, and it seems probable that another blend affects the centre of the line.

Thus our observations are consistent with the view that the surface of the Ap stars is wholly covered by a magnetic field whose strength varies within a limited range from place to place. This result is not entirely new (see e.g. Preston 1971b). But thanks to the high quality of our spectra (as compared to the photographic ones), much more stringent constraints can be set on the filling factor and on the field distribution. Even more importantly, given the large number of stars that have been studied, the abovementioned result can confidently be regarded as a generic property of Ap stars.

The much increased sample of stars with fairly to well studied magnetic field modulus (as compared with the data available in the literature so far) also allows one to derive statistically significant results, e.g. about the distribution of the strength and of the amplitude of variation of the fields.

An histogram illustrating the distribution of the average $\langle H\rangle_{\text {av }}$ of the mean magnetic field modulus is shown in Fig. 47. It is plotted from the values of $\langle H\rangle_{\text {av }}$ appearing in Table 2, with this quantity defined as explained in Sect. $4^{3}$. The shaded part of the histogram corresponds to the stars that have been observed throughout their whole rotation cycle; the unshaded area accounts for the stars for which only partial phase coverage has been achieved. The highest bin of the histogram is "open" on the high value side: it contains all stars for which $\langle H\rangle_{\text {av }}>15 \mathrm{kG}$.

\footnotetext{
$\overline{3}$ The use of $\langle H\rangle_{\text {av }}$ to characterize the strength of the magnetic field of the studied stars, even when the phase coverage achieved with the presently available observations is incomplete, is justified by the fact that the relative amplitudes of variation of the mean field modulus of most stars are small (see below).
} 


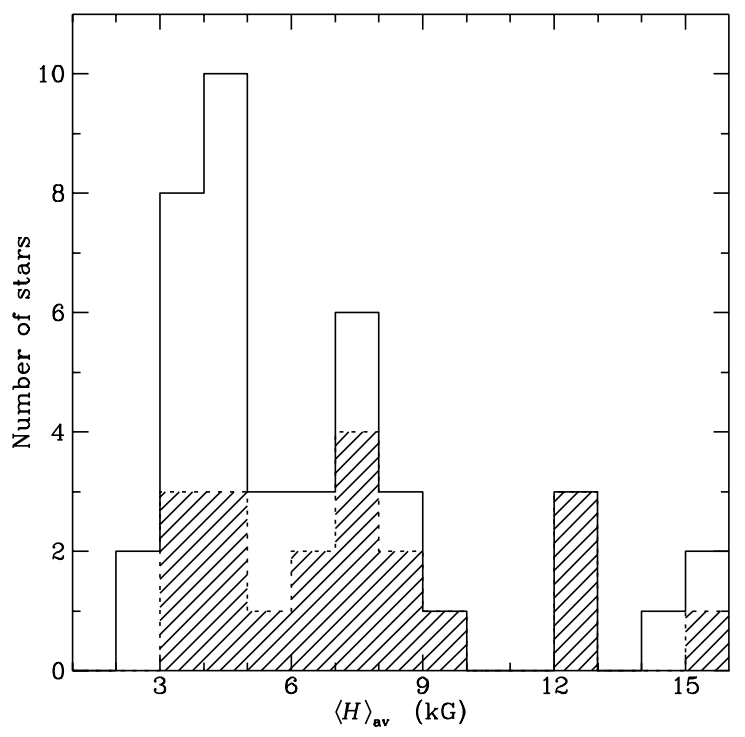

Fig. 47. Histogram showing the distribution of the phase-averaged mean magnetic field moduli of the 42 Ap stars with resolved magnetically split lines presently known. The shaded part of the histogram corresponds to those stars that have been observed throughout their rotation cycle (see text)

The vast majority of the studied stars have average magnetic field moduli in the range $3-9 \mathrm{kG}$. There are a few, more or less isolated stars, with considerably stronger fields. Babcock's star, HD 215441, more than 35 years after its discovery (Babcock 1960), still stands as the record holder, with its mean field modulus close to $34 \mathrm{kG}$. The next strongest fields hardly reach $17 \mathrm{kG}$.

But while the high field strength tail of the distribution seems fairly compatible with the combination of a "standard" statistical distribution (analogous to e.g. Poisson's law) and of small number statistics, the low field end shows a puzzling, rather sharp cutoff. Indeed, while more than $40 \%$ of the stars of the sample have a value of $\langle H\rangle_{\text {av }}$ between 3 and $5 \mathrm{kG}$, for only 2 stars (less than $5 \%$ ) is this quantity less than $3 \mathrm{kG}$. $\langle H\rangle_{\text {av }}$ in these 2 stars, HD 29578 and HD 75445, is resp. $2782 \mathrm{G}$ and $2985 \mathrm{G}$. Although it is not clear whether a full rotation cycle of those two stars has been sampled so far, it seems unlikely that their mean field modulus may at any phase go much below its lowest value found until now (resp. $2.7 \mathrm{kG}$ and $2.9 \mathrm{kG}$ : see Sects. 5.7 and 5.14). As a matter of fact, there is only one star, the SB2 HD 59435, for which some of our measurements of the mean field modulus yield values significantly lower (down to approx. 2.2 kG). But this star has one of the largest relative amplitudes of field modulus variation, and since our observations likely cover most of its rotation period (and in particular its mean field modulus minimum: see Sect. 5.10 and Fig. 19), it can be regarded as well established that the average over its rotation cycle of its mean field modulus is not smaller than $3 \mathrm{kG}$. As mentioned in Sect. 5.6, the profile of Fe II $\lambda 6149.2$ in
HD 18078 at phases where no splitting is observed is still consistent with a field modulus of the order of $3 \mathrm{kG}$.

Thus all the data gathered so far are consistent with the view that the average of the mean field modulus of Ap stars over their rotation cycle is never less than $2.8-3.0 \mathrm{kG}$. However, we expect to be able to observe resolved magnetically split lines for significantly weaker fields. Indeed, taking $10^{4} \mathrm{~K}$ as a representative temperature for an Ap star, the thermal broadening for iron is $1.7 \mathrm{~km} \mathrm{~s}^{-1}$. Microturbulence is not thought to be large in Ap stars: it is unlikely to exceed $2 \mathrm{~km} \mathrm{~s}^{-1}$. For the configuration most used in this study, the long camera of the ESO CES, the full width at half maximum of the instrumental profile is $3 \mathrm{~km} \mathrm{~s}^{-1}$. Combining these three contributions quadratically, it appears possible two resolve two lines (or two line components) separated by $4.0 \mathrm{~km} \mathrm{~s}^{-1}$. For the split components of Fe II $\lambda 6149.2$, this corresponds to a limit of resolution of $1.7 \mathrm{kG}$, in the absence of any broadening source other than those already considered. This latter restriction is realistic, as can be seen from the consideration of the stars with resolved lines studied here. The uniformity of the field of most of them (and the resulting sharpness of the split components of Fe II $\lambda 6149.2$ ) has been stressed above. Furthermore, rotational Doppler broadening is negligible in most cases: for a stellar radius of $5 R_{\odot}$ and a rotation period of $1 \mathrm{yr}$ (a value reached or exceeded by about half the stars with resolved lines known), $v \sin i$ is smaller than $0.7 \mathrm{~km} \mathrm{~s}^{-1}$. These $\operatorname{argu}-$ ments indicate that we should be able to detect magnetic line splitting in a non-negligible number of stars with a mean field modulus of significantly less than $3 \mathrm{kG}$. This view is also supported by the consideration of the splitting of Fe II $\lambda 6149.2$ in the spectrum of HD 75445 shown in Fig. 2 (corresponding to a mean field modulus of approx. $3 \mathrm{kG}$ ): clearly the components of the line could still be (partly) resolved even if the star had a considerably weaker field.

Thus one is led to the intriguing conclusion that the absence among the known stars with resolved magnetically split lines of any star with a phase-averaged field modulus lower than $2.8-3.0 \mathrm{kG}$ is not due to observational limitations but rather reflects an intrinsic stellar property. This is somewhat unexpected, since the distribution of the mean longitudinal field (the field moment that has been most determined) is continuous, strongly skewed toward small field values, down to the limit of detectability (Landstreet 1992).

Beside the stars discussed here, we have in the course of this project observed stars in which we failed to detect magnetically resolved lines. The detailed study of those (much more numerous) stars is beyond the scope of the present work. From a preliminary inspection of their spectra, it appears that their lines either are sufficiently broadened by rotational Doppler effect to smear out a possible splitting by a field 


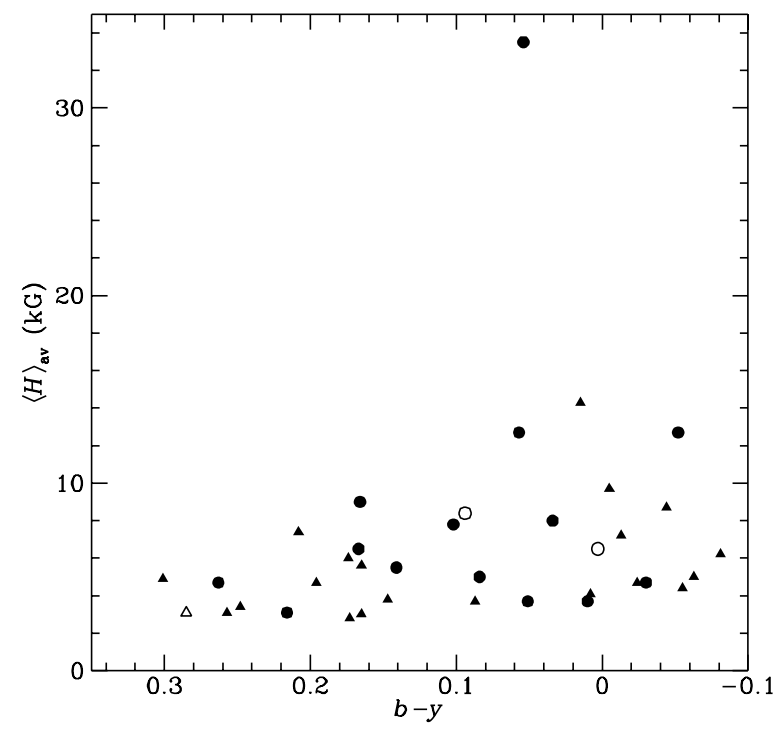

Fig. 48. Observed average of the mean magnetic field modulus against the Strömgren photometric index $(b-y)$. Dots: stars with field modulus determination throughout the rotation cycle; triangles: stars with incomplete phase coverage of the field modulus measurements. Open symbols are used to distinguish those stars for which the photometric index is affected by the contribution of a companion

of the order of $3 \mathrm{kG}$, or (like HD 133792, shown in Fig. 1) have profiles so sharp and "clean" that the considered star must have quite a weak field (probably not exceeding $1 \mathrm{kG}$ ), or perhaps even no field at all. In any case, there is no flagrant inconsistency with the existence of a sharp discontinuity in the distribution of the mean field moduli around $3 \mathrm{kG}$.

We shall not, for the time being, attempt to interpret this discontinuity, which is reported for the first time here. To address this question properly, we consider that it is necessary in a first stage to exploit more fully the information contained in our observations of stars with unresolved lines (in particular, by deriving more quantitative constraints on their magnetic fields). This will be the subject of a future work.

We shall now discuss possible correlations between the mean magnetic field modulus and other stellar properties.

In Fig. 48, the average $\langle H\rangle_{\mathrm{av}}$ of the field modulus is plotted against the Strömgren photometric index $(b-y)$. In the figure, different symbols are used to distinguish the stars for which full phase coverage is achieved (dots) from those where our data only sample part of the rotation cycle (triangles). Open symbols are used to single out three stars whose colour is distorted by the contribution of a companion: the SB2s HD 55719 and HD 59435, and the visual binary HD 81009 (a system discussed in some detail in Paper I, whose components of almost equal visual magnitude are separated by less than $0 . \prime 2$ ).

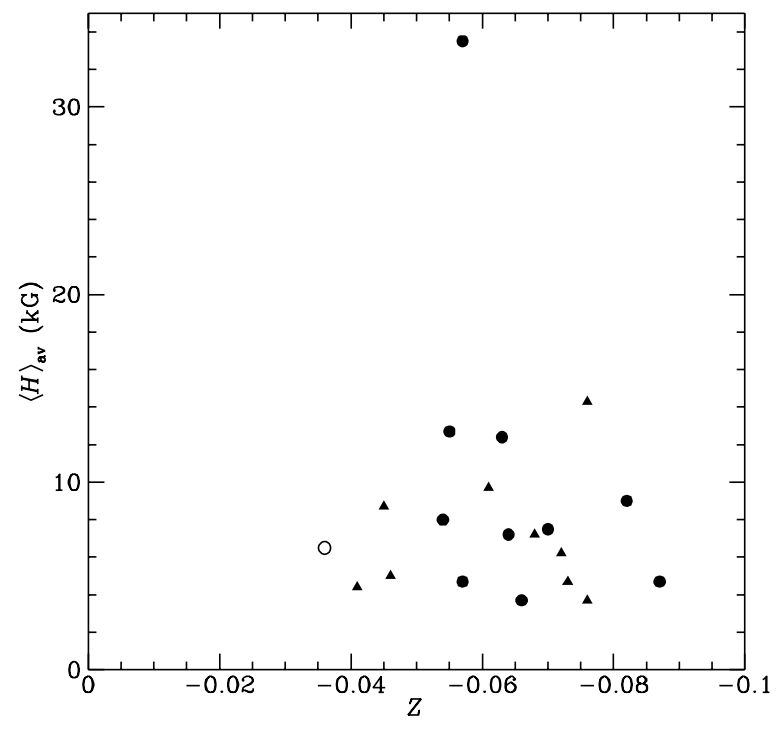

Fig. 49. Observed average of the mean magnetic field modulus against the Geneva photometric index $Z$. Dots: stars with field modulus determination throughout the rotation cycle; triangles: stars with incomplete phase coverage of the field modulus measurements. Open symbols are used to distinguish those stars for which the photometric index is affected by the contribution of a companion

$(b-y)$ is primarily a temperature indicator, in spite of the facts that it is affected by interstellar reddening and that the chemical peculiarities of Ap stars confer them anomalous colours. Therefore Fig. 48 essentially illustrates the relation between the mean magnetic field modulus and the stellar temperature. From its consideration, it appears that while the $\sim 3 \mathrm{kG}$ lower limit of the field strength distribution is roughly independent of the temperature, hotter stars may have stronger fields than cooler stars. In other words, the scatter of the possible intensities of the field modulus for a given temperature increases with the temperature. The inconsistency between this conclusion and the location of HD 215441 (the star with the strongest magnetic field known) in Fig. 48 is only apparent and due to our neglecting the fairly strong reddening of this star (see e.g. Stepien 1968). It is actually one of the very hottest stars of the whole sample, if not the hottest one: if its intrinsic (and not apparent) colour was used for the plot, it would appear very close to the right edge.

The indications found here that hot Ap stars may achieve higher magnetic field moduli than cool Ap stars may bear some relation with the result that the longitudinal fields of hot He weak stars and of He strong stars are in the average stronger than those of Ap stars and of cool He weak stars (Landstreet 1992). However, it must be stressed that our sample is limited to stars of the latter groups, and does not encompass any hot He weak or $\mathrm{He}$ strong star (which, as a matter of fact, are too hot to show the line Fe II $\lambda 6149.2)$. 


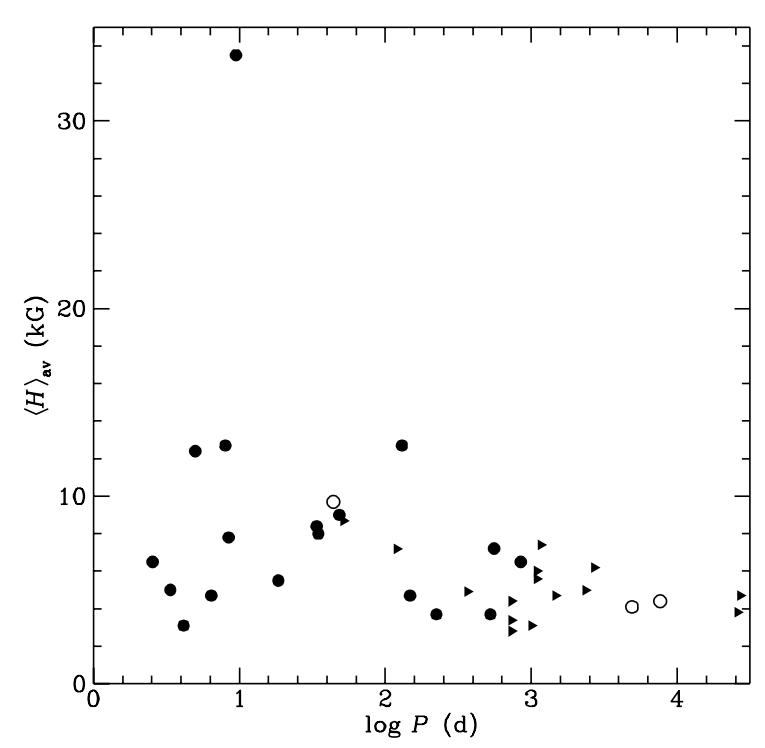

Fig. 50. Observed average of the mean magnetic field modulus against rotation period. Dots: stars with well determined periods (open dots are used to distinguish those stars for which our field modulus measurements do not cover the whole rotation cycle); triangles: stars for which only a lower limit of the period is known

Another photometric quantity of interest is the reddening-free index $Z$ of Geneva photometry. Cramer \& Maeder (1981) had suggested the existence of a linear relation between $Z$ and $\langle H\rangle$ for stars earlier than A5 (that is, with the Geneva index $Y>-0.110$ ) with a field modulus not exceeding $5 \mathrm{kG}$. This result has been questioned by various authors (see in particular Oetken 1984). The most recent discussion of the issue (Paper II) did not support its validity. However, the discussion relied to some extent on indirect determinations of the field modulus, from the differential magnetic intensification of the Fe II lines $\lambda 6147.7$ and $\lambda 6149.2$. The relation between the latter and $\langle H\rangle$ appears at present more ambiguous than it seemed from the consideration of the much smaller number of data available when Paper II was written (see also Takeda 1991), so that it will have to be rediscussed. Because this discussion calls on a number of considerations that go beyond the scope of the present paper, and because it is especially relevant to the study of the stars whose lines are not magnetically resolved, we leave it for a future work in which those aspects will be addressed.

Therefore, the results reported in this paper are insufficient to settle the question of the possible relation between $Z$ and $\langle H\rangle$, since a large fraction of the stars for which new data have been obtained are either too cool or too strongly magnetic to fall within the purported range of validity of that relation. For the sake of completeness, we show in Fig. 49 a plot of the mean magnetic field modulus against the $Z$ parameter. As for the dependence on $(b-y)$, but somewhat more marginally, there is some in- dication that the field strengths can span a wider range (or have a higher upper limit) for higher absolute value of $Z$. Also the absence of any star with $|Z|<0.030$ is noticeable. But these two results must be taken cautiously: the sample of stars in which we have looked for resolved lines is likely significantly biased toward high absolute values of $Z$, since in a first stage of our study, such a high value was one of the criteria used to select some of the candidates to be observed.

There also seems to be some anticorrelation between the mean magnetic field modulus and the stellar rotation period. This is illustrated in Fig. 50, where the observed average of the field modulus, $\langle H\rangle_{\mathrm{av}}$, is plotted against the rotation period $P$. For stars for which only a lower limit of the latter has been derived so far, this lower limit is shown. 35 stars appear in Fig. 50 (not enough is known about the period of the 7 remaining stars), of which 16 have a rotation period shorter than 150 days. Of these 16 stars, 10 have $\langle H\rangle_{\text {av }}>7.5 \mathrm{kG}$, while the 19 stars with a period longer than $150 \mathrm{~d}$ all have $\langle H\rangle_{\mathrm{av}}<7.5 \mathrm{kG}$. In order to check whether this might result from a possible correlation between the effective temperature and the rotation period, we have in Fig. 51 plotted $P$ against $(b-y)$ for the stars of our sample. From this figure, period and temperature appear essentially independent from each other.

Let us now discuss the variations of the mean field modulus, and their meaning in terms of geometrical structure of the studied fields.

Of all the stars for which the observations obtained until now sample adequately the variations of the mean magnetic field modulus, only one (HD 208217) has a doublewaved variation curve, while all others only have one minimum and one maximum per rotation cycle. This indicates that the field of these stars is generally not a centred dipole. Indeed for such a dipole, the field would be weakest at the magnetic equator and strongest at the rotation poles, and would have the same intensity at both of those poles. Provided that the geometry of the observation is such that both poles come alternatively into view ${ }^{4}$, there should be two (identical) maxima and two (identical) minima of the field modulus per rotation period. Statistically, in a sample of the size studied here, there should be several stars fulfilling the condition $(i+\beta)>90^{\circ}$ : that the magnetic field of Ap stars is not a centred dipole, a result already clear for a number of stars, is established here much more generally. This is even more so because in HD 208217, the two field maxima appear different: the field is not a centred dipole either, in spite of the doublewave variation.

As a matter of fact, many of the $\langle H\rangle$ variation curves show some anharmonicity. This indicates that the fields

\footnotetext{
$4 \quad$ That is, provided that the sum of the angles $i$ (between the stellar rotation axis and the line of sight) and $\beta$ (between the magnetic and rotation axes) is greater than $90^{\circ}$.
} 


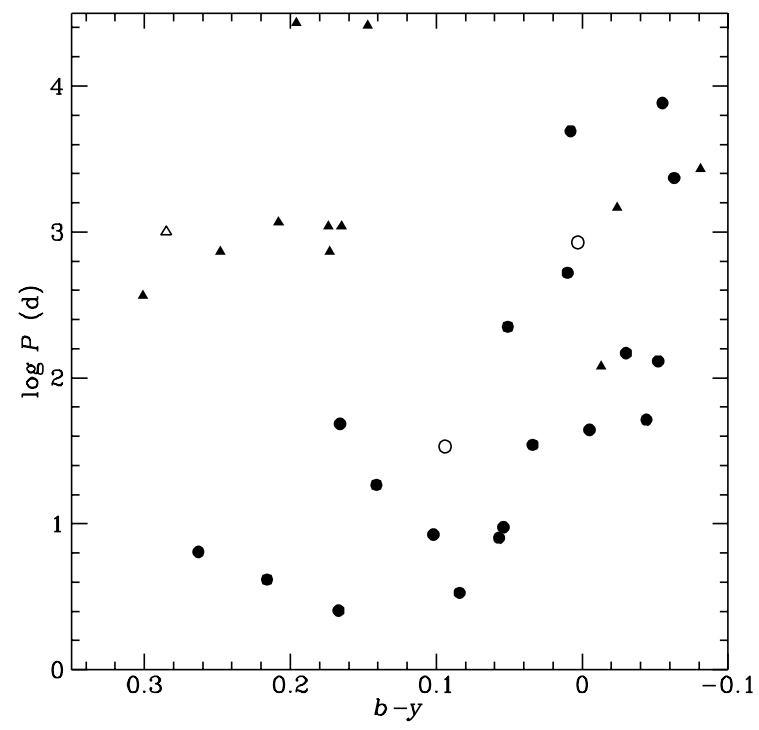

Fig. 51. Rotation period of the stars with magnetically resolved lines against the Strömgren photometric index $(b-y)$. Dots: stars with well determined periods; triangles: stars for which only a lower limit of the period is known. Open symbols are used to distinguish those stars for which the photometric index is affected by the contribution of a companion

of the corresponding stars have significant non-dipolar components (e.g., higher-order multipoles). Several of the $\langle H\rangle$ curves (HD 137909, HD 142070, probably HD 94660 and HD 187474) are not even symmetric about the phases of their extrema. This means that their fields do not have the property of cylindrical symmetry with respect to an axis passing through the centre of the star. This conclusion is the same as reached by Mathys (1993) from the consideration of other field moments ${ }^{5}$ in other, (generally) faster-rotating Ap stars: again, the present study confirms that a result (the lack of symmetry of the magnetic field) that could in the past be suspected to belong only to some isolated, possibly exceptional object, is in fact widespread among magnetic Ap stars.

The distribution of the ratio $q=\langle H\rangle_{\max } /\langle H\rangle_{\min }$ of the maximum to the minimum of the mean magnetic field modulus (hereafter called the relative amplitude of the variation) is shown in Fig. 52. Again, the shaded part of the histogram corresponds to "real" extrema (that is, to stars observed throughout their whole rotation cycle). Both the complete histogram and the shaded part are strongly skewed toward small values of the ratio: this consistency suggests that few of the stars for which phase coverage is still incomplete will be found to have a large relative amplitude of variation of $\langle H\rangle$. This, however, makes sense only provided that there is no relation between the

5 Note also that the curves of variation of the longitudinal field of HD 81009 and of HD 126515 are not symmetric about their extrema, although the field modulus curves of those stars have that symmetry (see Sects. 5.15 and 5.22).

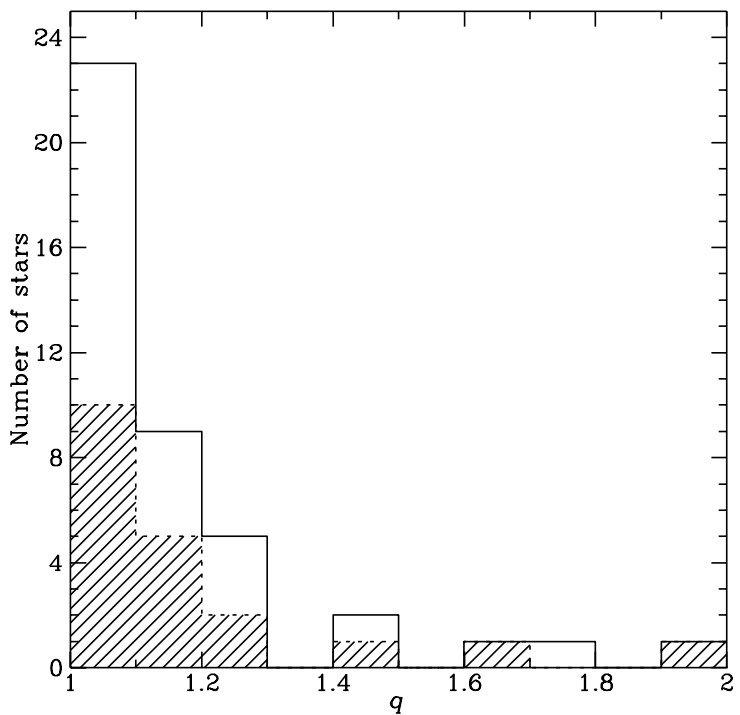

Fig. 52. Histogram showing the distribution of the relative amplitudes of variation $q=\langle H\rangle_{\max } /\langle H\rangle_{\min }$ of the mean magnetic field modulus of the $42 \mathrm{Ap}$ stars with resolved magnetically split lines presently known. The shaded part of the histogram corresponds to those stars that have been observed throughout their rotation cycle. For stars for which observations well distributed in phase have not been obtained yet (unshaded part of the histogram), a lower limit of $q$ is used

rotation period and the variation amplitude (since the stars with incomplete phase coverage have periods longer in the average than the periods of the stars for which our data sample well the rotation cycle). The possible existence of such a correlation, although it does not seem very likely (cf. the large amplitude of variation of the shortperiod star HD 65339), cannot be definitely ruled out at present. On the other hand, it can be noted that for 7 at least of the stars with resolved magnetically split lines currently known, $q$ is greater than 1.25 , that is, the upper limit for a centred dipole.

Knowing the mean field modulus alone is insufficient to characterize the geometrical structure of the stellar magnetic field. The latter can be better (although not uniquely) constrained from the simultaneous consideration of the field modulus and of the longitudinal field, and of their variation along the star's rotation cycle. As can be seen in Table 2, measurements of the longitudinal field throughout the rotation period are available for 12 of the stars with magnetically resolved lines currently known. For all of them but two (HD 9996 and HD 187474), enough determinations of the field modulus have been performed to define its variations. In Fig. 53, we have plotted the ratio $q$ of the extrema of the field modulus against $r$, the quantity commonly used to characterize the relative amplitude of variation of the longitudinal field. The latter is defined as $r=\left\langle H_{\mathrm{z}}\right\rangle_{\mathrm{s}} /\left\langle\mathrm{H}_{\mathrm{z}}\right\rangle_{\mathrm{l}}$, where $\left\langle H_{\mathrm{z}}\right\rangle_{\mathrm{s}}$ is the smaller 


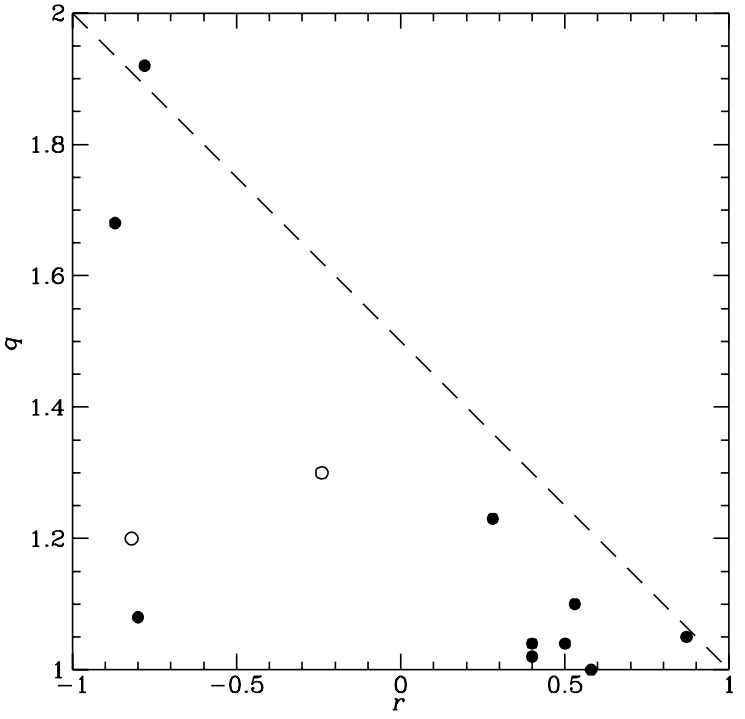

Fig. 53. Relative amplitude of variation $q$ of the mean magnetic field modulus against the ratio of the extrema of the mean longitudinal magnetic field. Open symbols are used to distinguish two stars, HD 9996 and HD 187474, for which only incomplete phase coverage is achieved by our measurements of the field modulus

and $\left\langle H_{z}\right\rangle_{1}$ the larger (in absolute value) of the observed extrema $\left\langle H_{z}\right\rangle_{\max }$ and $\left\langle H_{z}\right\rangle_{\min }$ of $\left\langle H_{z}\right\rangle . r$ is negative when the longitudinal field reverses its sign, that is (in the simple picture of dipolar field geometry) when both magnetic poles come into view during a rotation period. $r$ gets close to -1 when the maximum and the minimum of $\left\langle H_{\mathrm{z}}\right\rangle$ are symmetric with respect to 0 , which in the dipole model occurs when the angle $\beta$ between the dipole axis and the stellar rotation axis is close to $90^{\circ}$. Conversely, for positive values of $r$, the same magnetic pole remains within view throughout the whole rotation cycle. $r$ tends towards 1 whenever the line of sight or the magnetic axis (or both) become parallel to the rotation axis.

The representative points of all the stars for which the relevant information exists are found in the lower left half of Fig. 53. This is emphasized on the figure by the dashed line crossing it diagonally. Thus, when the same magnetic pole is seen at all phases, the relative amplitude of variation of the mean field modulus is small. It seems that large variations of $\langle H\rangle$ can be observed only provided that both magnetic poles come within view at some phase. This is not trivial, as for a centred dipole, the field on the surface of the star is maximum at both poles and minimum at the equator. Even though this result is based upon a rather small number of stars, it points towards the rather general existence of a quite significant difference between the two magnetic poles of Ap stars.

From the simultaneous consideration of the curves of variation of the longitudinal field and of the mean field modulus, one can in principle derive uniquely a simple model of the magnetic field structure (e.g., dipole offset along its axis or superposition of collinear dipole and quadrupole), provided that the extrema of $\left\langle H_{z}\right\rangle$ and of $\langle H\rangle$ occur at the same phases, and that the curves of variations of both quantities are symmetric with respect to the phases of these extrema. We have shown above that those two conditions are generally not fulfilled. Thus more complex field models are required: we postpone their discussion to a future paper.

\subsection{Rotation periods}

As a by-product of the present study, we have been able to perform or to improve the determination of the rotation period of a number of stars, and to set lower limits on the period of several others. Consequently, the number of Ap stars known to rotate with periods longer than 30 days has almost doubled, rising from 16 to 31 (assuming that the fact that no variations of the magnetic field of HD 177765 have been observed so far is due to its having a long period). This allows a better characterization of the slow-rotation tail of the period distribution of those stars, as shown in Fig. 54. The shaded part of the histogram corresponds to those stars whose period is known, the unshaded one to stars for which only a lower limit is available so far. The upper bin of the histogram is "open" towards the large values of the period. Note however that there is currently no star showing definite evidence of having a period that would exceed its upper boundary, if it had the same width as the other bins (that is, if its upper boundary was 84 years).

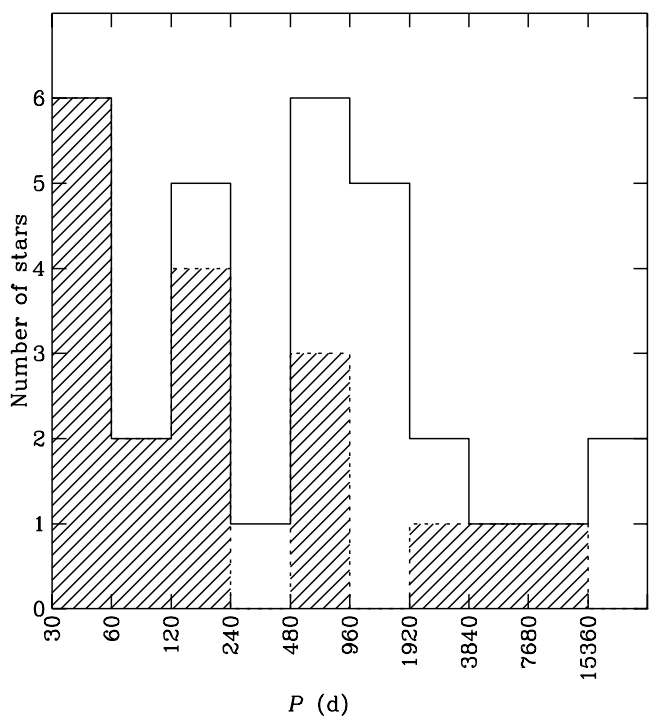

Fig. 54. Histogram showing the distribution of the rotation periods of Ap stars above 30 days. The shaded part of the histogram corresponds to stars whose actual rotation period is known; the unshaded part to stars for which only a lower limit of the period has been set so far 
Taking into account the fact that the stars for which only lower limits of the period have been obtained so far may, when they have been studied long enough, shift to higher bins in the histogram, the distribution in Fig. 54 is consistent with an equipartition of the long periods (say, over 1 year) of Ap stars on a logarithmic period scale, or in other words, with a distribution decreasing exponentially with the period. Nevertheless, this result at present still has a limited statistical significance, and it will be very interesting (but it will require quite a long time) to see if it remains valid as new long periods are determined. But it is already clear that periods in excess of one year, albeit infrequent, are not exceptional among Ap stars. It has sometimes been questioned in the past whether these periods do indeed correspond to stellar rotation, or if they rather belong to intrinsic, secular field variations. Leroy et al.'s (1994) study of $\gamma$ Equ (with HD 137949 the Ap star with the largest lower limit of the period until now) in linear polarization strongly favours the former interpretation. It should also be stressed that extremely slowly rotating Ap stars do not seem to differ systematically from their faster counterparts in any other respect (note in particular the absence of temperature dependence for the stars of the present sample in Fig. 51). Accordingly, the mechanisms by which stellar rotation can be slowed down to the extent observed in some Ap stars remain enigmatic.

At the opposite extreme, a few of the Ap stars with magnetically resolved lines have periods of the order of one week or less. Their interest comes not from their period by itself (the vast majority of Ap stars have period in the 1-7 day range), but rather from its conjunction with the appearance of resolved magnetically split lines. Indeed, the fact that rotational Doppler effect is small with respect to the magnetic splitting implies that the inclination of the rotation axis with respect to the line of sight must be (very) small. In other words, these fast rotating stars with magnetically resolved lines are unique examples of stars definitely seen (rotation) pole on.

\subsection{Binarity and pulsation}

Accurate radial velocity determinations can be performed from the high-resolution spectra obtained for the present study of the magnetic fields. Thanks to this, and to the fact that we had to observe repeatedly the same stars over long time spans, we could report in this paper the occurrence of radial velocity variations in 8 stars for which such variability was not mentioned in Renson's et al. (1991) General Catalogue of Ap and Am stars. Our observations also confirmed the indication given in that catalogue that the radial velocity of HD 94660 is variable. In addition, 7 of the stars considered here belong to well studied spectroscopic binaries, HD 81009 is a visual binary system (discussed to some extent in Paper I), and HD 165474 is the secondary of a visual binary whose primary ( $7^{\prime \prime}$ apart) is the fast-rotating, normal A star HD 165475. The detailed presentation of our radial velocity data and the derivation of the orbital elements of the binary systems are beyond the scope of this paper. For the time being, we shall restrict ourselves to a brief discussion of some intriguing facts that are unveiled through a first inspection of the measurements.

Of the 41 stars with resolved magnetically split lines that have been repeatedly observed (that is, excluding HD 47103), 18 appear to belong to binaries. This rate of occurrence of binarity (44\%) is between the frequencies found by Gerbaldi et al. (1985) for $\mathrm{SrCrEu}$ stars (46\%) and for hot Ap stars (37\%). However, 16 of the $18 \mathrm{Ap} \mathrm{bi-}$ naries considered here have an orbital period longer than $100 \mathrm{~d}$, and for at least 10 of them, the orbital period must even exceed $1000 \mathrm{~d}$. Thus the distribution of the orbital periods of the binary systems containing an Ap star with magnetically resolved lines is much more skewed towards long periods than that of Ap binaries in general (see Fig. 8 of Gerbaldi et al. 1985).

The interpretation of this observational result is not straightforward. Components of wide binaries like those of interest here must have evolved in an essentially independent manner. In particular, the slow rotation of the Ap star cannot be attributed to an interaction with its companion. As a matter of fact, there is no relation between the rotation and the orbital periods of the stars of interest: for 6 of them the rotation period is shorter than the orbital period, while for another 6 , the orbital period is longer (for the 6 remaining systems, the data available so far are inconclusive).

An alternative explanation is that, rather than an $e x-$ cess of long orbital periods among binaries containing Ap stars with magnetically resolved lines, what we observe in fact is a deficiency of short orbital periods among such binaries. In support of this idea, one may note that the binary nature of several of the stars with resolved lines has been established from the observation of very small radial velocity changes (of a few $\mathrm{km} \mathrm{s}^{-1}$ ) which have taken place over quite long timescales (several years). The detection of such minute variations was possible only because over a long period we repeatedly obtained high-resolution spectra of very slowly rotating stars (which allowed us to derive very accurate radial velocities). For the vast majority of Ap stars, observations have not been performed in such favourable conditions: therefore we conjecture that a significant number of Ap binaries with very long orbital periods may have been omitted from Gerbaldi et al.'s (1985) study. If this assumption is correct, the frequencies of binary systems containing Ap stars obtained by Gerbaldi et al. (1985) are underestimated. Then the occurrence of binarity among Ap stars with magnetically resolved lines would be lower than in Ap stars in general, due to a lack of short orbital periods for stars with resolved lines. This would seem to indicate that Ap stars cannot achieve slow enough rotation to show resolved magnetically split lines if they belong to (fairly) close binaries. 
Another remarkable result of the present study is that 6 of the 42 Ap stars with magnetically resolved lines belong to the group of the rapidly oscillating Ap (roAp) stars (e.g., Kurtz 1990). To some extent, this results from a selection bias, since due to the interest of some of us in roAp stars (and especially in their magnetic field), we have systematically checked all of them for possible resolution of magnetically split lines. Nevertheless, the fraction of stars showing magnetically resolved lines appears much higher among roAp stars ( 6 out of 28 roAp stars presently known) than among Ap stars in general. There is no obvious explanation for this. Let us just note that one may possibly draw some parallel between the low frequency of binaries among Ap stars with resolved lines (according to the hypothesis developed above) and the absence of spectroscopic binaries among roAp stars (Hubrig \& Mathys 1996).

\section{Conclusion}

Within the course of the present project, we have discovered so far 29 Ap stars with resolved magnetically split lines (16 of which are reported for the first time in this paper). This raises the number of such stars presently known to 42 , compared to 12 when we started this study (the 42nd star with magnetically resolved lines has been discovered more recently by Babel et al. 1995). As of end of August 1995, we had performed more than 750 measurements of the mean magnetic field modulus of 40 of those 42 stars (compared to about 80 measurements in the literature at the time of the beginning of our investigation). Those measurements, in the most favourable cases (more than one third of the studied stars), reach an unprecedented accuracy $(25-30 \mathrm{G})$. Our study brought the number of stars with field modulus measurements sampling (fairly) well the rotation cycle from 4 to 16 . For a few more stars good phase coverage should soon be achieved. These achievements allowed us to discuss various aspects of the magnetism of Ap stars in greater generality and on sounder statistical grounds than had previously been possible.

As part of our search for resolved magnetically split spectral lines in Ap stars, we have, of course, achieved a large number of negative results. These are not without interest (in particular, they can still be used to derive constraints on the magnetic fields of the considered stars, as well as on their projected equatorial velocity). Their analysis will undoubtedly allow us to complement and to refine some of the results reported here, and we intend to devote a future publication to their presentation.

In the future, we aim at completing progressively the phase coverage of the variations of the field modulus of the stars with resolved lines for which the measurements presented in this paper sample only partly the rotation period. Particular attention is paid to the very slowly rotating stars, for which a good knowledge of the field may prove essential for the understanding of the braking mechanisms that must have been (and possibly still are) at work. Since the simultaneous consideration of the variations of the field modulus and of the longitudinal field is required to derive meaningful constraints on the field geometrical structure, and since enough determinations of $\left\langle H_{\mathrm{z}}\right\rangle$ are available at present for less than a dozen stars with magnetically resolved lines, we have also undertaken a systematic study of the longitudinal field of the other stars of that type.

Acknowledgements. This research has made use of the Simbad database, operated at CDS, Strasbourg, France. This work was done partly during a stay of $\mathrm{SH}$ at the European Southern Observatory (Santiago, Chile), as ESO Senior Visiting Scientist.

\section{References}

Abt H.A., Willmarth D.W., 1994, ApJS 94, 677

Adelman S.J., 1974, ApJS 28, 51

Adelman S.J., 1981, A\&AS 44, 265

Albrecht R., Jenkner H., Weiss W.W., Wood H.J., 1977, A\&A 58, 93

Babcock H.W., 1947, ApJ 105, 105

Babcock H.W., 1954, ApJ 120, 66

Babcock H.W., 1958, ApJS 3, 141

Babcock H.W., 1960, ApJ 132, 521

Babcock H.W., 1967, in: The magnetic and related stars, Cameron R.C. (ed.). Mono Book Corporation, Baltimore, p. 551

Babel J., North P., Queloz D., 1995, A\&A 303, L5

Baranne A., Queloz D., Mayor M., et al., 1996, OHP preprint No. 94

Bohlender D.A., Landstreet J.D., Thompson I.B., 1993, A\&A 269,355

Bonsack W.K., 1976, ApJ 209, 160

Borra E.F., Landstreet J.D., 1975, PASP 87, 961

Borra E.F., Landstreet J.D., 1977, ApJ 212, 141

Catalano F.A., Leone F., 1990, A\&AS 83, 491

Cramer N., Maeder A., 1981, Photometric classification of B and Ap stars with an application to 3600 stars. In: Upper main sequence chemically peculiar stars, 23rd Liège Astrophys. Coll., p. 61

D’Odorico S., Avila G., Molaro P., 1989, The Messenger 58, 58 Dekker H., D'Odorico S., Fontana A., 1994, The Messenger 76, 16

Deul E.R., van Genderen A.M., 1983, A\&A 118, 289

Didelon P., 1987, The Messenger 49, 5

Didelon P., 1988, Surface magnetic field measurements in hot chemically peculiar stars. In: The impact of very high $S / N$ spectroscopy on stellar physics, IAU Symp. No. 132, Cayrel de Strobel G., Spite M. (eds.). Kluwer, Dordrecht, p. 313

Dworetsky M.M., 1982, Observatory 102, 138

Gerbaldi M., Floquet M., Hauck B., 1985, A\&A 146, 341

Gillet D., Burnage R., Kohler D., et al., 1994, A\&AS 108, 1

Glagolevskij Yu.V., Bychkov V.D., Iliev I.Kh., Romanyuk I.I., Chunakova N.M., 1982, SvAL 8, 12

Glagolevskij Yu.V., Bychkov V.D., Romanyuk I.I., Chunakova N.M., 1986, Bull. Spec. Astrophys. Obs. 19, 26 
Glaspey J., 1993, User's Manual for GECKO - The Coudé F/4 Spectrograph

Heck A., Mathys G., Manfroid J., 1987, A\&AS 70, 33

Hensberge H., 1993, Long term variability in CP stars. In: Peculiar versus normal phenomena in A-type and related stars, IAU Coll. No. 138, Dworetsky M.M., Castelli F., Faraggiana R. (eds.). Astron. Soc. Pac. Conf. Ser. 44, 547

Hubrig S., Mathys G., 1996 (in preparation)

Huchra J., 1972, ApJ 174, 435

Kreidl T.J., 1985, IBVS 2739

Kreidl T.J., Kurtz D.W., Schneider H., et al., 1994, MNRAS 270,115

Kurtz D.W., 1983, MNRAS 202, 1

Kurtz D.W., 1989, MNRAS 238, 261

Kurtz D.W., 1990, ARA\&A 28, 607

Kurtz D.W., 1991, MNRAS 249, 468

Kurtz D.W., Martinez P., 1987, MNRAS 226, 187

Landstreet J.D., 1980, AJ 85, 611

Landstreet J.D., 1982, ApJ 258, 639

Landstreet J.D., 1988, ApJ 326, 967

Landstreet J.D., 1992, A\&AR 4, 35

Landstreet J.D., Barker P.K., Bohlender D.A., Jewison M.S., 1989, ApJ 344, 876

Leeman S., 1964, Mon. No. Astr. Soc. Sth Af. 23, 6

Leroy J.L., 1995a, A complete magnetic model of beta Corona Borealis. In: La polarimétrie, outil pour l'étude de l'activité magnétique solaire et stellaire, Mein N., Sahal S. (eds.). Observatoire de Paris-Meudon (in press)

Leroy J.L., 1995b, A\&AS 114, 79

Leroy J.L., Bagnulo S., Landolfi M., Landi Degl'Innocenti E., 1994, A\&A 284, 174

Leroy J.L., Landolfi M., Landi Degl'Innocenti M., et al., 1995, A\&A 301, 797

Lindgren H., Gilliotte A., 1989, The Coudé Echelle Spectrometer - The Coudé Auxiliary Telescope, ESO Operating Manual No. 8

Manfroid J., Mathys G., 1996, A\&AS (submitted)

Manfroid J., Renson P., 1983, Inf. Bull. Variable Stars 2311

Martinez P., 1993, Ph.D. Thesis, University of Cape Town

Martinez P., Kurtz D.W., 1994, MNRAS 271, 118

Martinez P., Kurtz D.W., Meintjes P.J., 1993, MNRAS 260, 9

Mathys G., 1988, A\&A 189, 179

Mathys G., 1989, Fundam. Cosmic Phys. 13, 143

Mathys G., 1990, A\&A 232, 151 (Paper I)

Mathys G., 1991, A\&AS 89, 121

Mathys G., 1993, Magnetic field diagnosis through spectropolarimetry. In: Peculiar versus normal phenomena in A-type and related stars, IAU Coll. No. 138, Dworetsky M.M., Castelli F., Faraggiana R. (eds.). Astron. Soc. Pac. Conf. Ser. 44, 232

Mathys G., 1994a, The Coudé Echelle Spectrometer — Update to the Operating Manual, ESO

Mathys G., 1994b, A\&AS 108, 547

Mathys G., 1995a, A\&A 293, 733

Mathys G., 1995b, A\&A 293, 746
Mathys G., Hubrig S., 1996 (in preparation)

Mathys G., Lanz T., 1992, A\&A 256, 169 (Paper II)

Mathys G., Solanki S.K., 1989, A\&A 208, 189

Mathys G., Landstreet J.D., Lanz T., 1993, Observations of magnetically split lines in Ap stars. In: Peculiar versus normal phenomena in A-type and related stars, IAU Coll. No. 138, Dworetsky M.M., Castelli F., Faraggiana R. (eds.). Astron. Soc. Pac. Conf. Ser. 44, 300 (Paper III)

Neubauer F.J., 1944, ApJ 99, 134

North P., 1987, A\&AS 69, 371

North P., 1994, in: The 25th workshop and meeting of European working group on CP stars, Jankovics I., Vincze I.J. (eds.). Gothard Astrophysical Observatory of Eötvös University, Szombathely, Hungary, p. 3

North P., Adelman S.J., 1995, A\&AS 111, 41

North P., Cramer N., 1981, Ap stars detected in open clusters by the Geneva photometry. In: Upper main sequence chemically peculiar stars, 23rd Liège Astrophys. Coll., p. 55

Oetken L., 1984, Astron. Nachr. 306, 187

Preston G.W., 1969a, ApJ 156, 967

Preston G.W., 1969b, ApJ 157, 247

Preston G.W., 1969c, ApJ 158, 1081

Preston G.W., 1970, ApJ 160, 1059

Preston G.W., 1971a, ApJ 164, 309

Preston G.W., 1971b, PASP 83, 571

Preston G.W., Wolff S.C., 1970, ApJ 160, 1071

Renson P., Gerbaldi M., Catalano F.A., 1991, A\&AS 89, 429

Rice J.B., 1988, A\&A 199, 299

Scholz G., 1978, Astron. Nachr. 299, 81

Scholz G., 1979, Astron. Nachr. 300, 213

Scholz G., 1983, ApSS 94, 159

Scholz G., Lehmann H., 1988, Astron. Nachr. 309, 33

Stepień K., 1968, ApJ 154, 945

Sugar J., Corliss C., 1985, Atomic Energy Levels of the IronPeriod Elements: Potassium through Nickel, J. Phys. Chem. Ref. Data 14, Suppl. 2

Takeda Y., 1991, PASJ 43, 823

van den Heuvel E.P.J., 1971, A\&A 11, 461

Wade G.A., 1995, On the magnetic field of $\beta$ Coronae Borealis (preprint)

Wade G.A., Elkin V.G., Landstreet J.D., et al., 1996a, A\&A (in press - ESO preprint No. 1142)

Wade G.A., North P., Mathys G., Hubrig S., 1996b, A\&A (in press)

Waelkens C., 1985, A\&AS 61, 127

Willmarth D., 1996, Instrumentation Operation Manual, Coudé Spectrograph, Kitt Peak National Observatory

Wolff S.C., 1969, ApJ 158, 1231

Wolff S.C., 1975, ApJ 202, 127

Wolff S.C., Morrison N.D., 1973, PASP 85, 141

Wolff S.C., Wolff R.J., 1970, ApJ 160, 1049

Zijlstra A.A., Giraud E., Melnick J., Dekker H., D'Odorico S., 1996, EMMI \& SUSI - The ESO Multi-Mode Instrument and The Superb Seeing Imager, ESO Operating Manual No. 15, Version No. 3.0 